\title{
Probes and Tests of Strong-Field Gravity with Observations in the Electromagnetic Spectrum
}

\author{
Dimitrios Psaltis \\ Physics and Astronomy Departments \\ University of Arizona \\ Tucson, AZ 85721 \\ U.S.A. \\ email: dpsaltis@physics.arizona.edu \\ http://www.physics.arizona.edu/ dpsaltis \\ Living Reviews in Relativity \\ ISSN 1433-8351
}

Accepted on 1 July 2008

Published on 18 November 2008

\begin{abstract}
Neutron stars and black holes are the astrophysical systems with the strongest gravitational fields in the universe. In this article, I review the prospect of using observations of such compact objects to probe some of the most intriguing general relativistic predictions in the strong-field regime: the absence of stable circular orbits near a compact object and the presence of event horizons around black-hole singularities. I discuss the need for a theoretical framework, within which future experiments will provide detailed, quantitative tests of gravity theories. Finally, I summarize the constraints imposed by current observations of neutron stars on potential deviations from general relativity.
\end{abstract}

This review is licensed under a Creative Commons Attribution-Non-Commercial-NoDerivs 3.0 Germany License. http://creativecommons.org/licenses/by-nc-nd/3.0/de/ 


\section{Imprint / Terms of Use}

Living Reviews in Relativity is a peer reviewed open access journal published by the Max Planck Institute for Gravitational Physics, Am Mühlenberg 1, 14476 Potsdam, Germany. ISSN 1433-8351.

This review is licensed under a Creative Commons Attribution-Non-Commercial-NoDerivs 3.0 Germany License: http://creativecommons.org/licenses/by-nc-nd/3.0/de/

Because a Living Reviews article can evolve over time, we recommend to cite the article as follows:

Dimitrios Psaltis,

"Probes and Tests of Strong-Field Gravity with Observations in the Electromagnetic Spectrum",

Living Rev. Relativity, 11, (2008), 9. [Online Article]: cited [<date $>$, http://www.livingreviews.org/lrr-2008-9

The date given as $<$ date $>$ then uniquely identifies the version of the article you are referring to.

\section{Article Revisions}

Living Reviews supports two different ways to keep its articles up-to-date:

Fast-track revision A fast-track revision provides the author with the opportunity to add short notices of current research results, trends and developments, or important publications to the article. A fast-track revision is refereed by the responsible subject editor. If an article has undergone a fast-track revision, a summary of changes will be listed here.

Major update A major update will include substantial changes and additions and is subject to full external refereeing. It is published with a new publication number.

For detailed documentation of an article's evolution, please refer always to the history document of the article's online version at http: //www.livingreviews.org/lrr-2008-9. 


\section{Contents}

1 Introduction $\quad 5$

2 The motivation for strong-field tests $r$

3 Astrophysical and Cosmological Settings of Strong Gravitational Fields 9

3.1 When is a gravitational field strong? . . . . . . . . . . . . . . . . . . . . . 9

3.2 A parameter space for tests of gravity . . . . . . . . . . . . . . . . . . . . . . . . 10

3.3 Probing versus testing strong-field gravity . . . . . . . . . . . . . . . . . . 14

4 Probing Strong Gravitational Fields with Astrophysical Objects $\quad 17$

4.1 Black-hole images . . . . . . . . . . . . . . . . . . . . . . 17

4.2 Continuum spectroscopy of accreting black holes . . . . . . . . . . . . . . . . 20

4.2.1 Luminosities of black holes in quiescence and the absence of a hard surface 20

4.2.2 Hard X-ray spectra of luminous black holes and the presence of an event

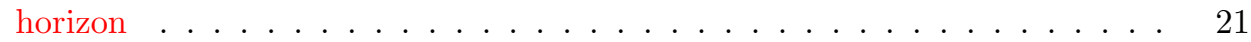

4.2.3 Measuring the radii of the innermost stable circular orbits of black holes using continuum spectra . . . . . . . . . . . . . . . . . . . 21

4.3 Line spectroscopy of accreting compact objects . . . . . . . . . . . . . . . . . . . . 24

4.3.1 Atomic lines from the surfaces of neutron stars . . . . . . . . . . . . . 24

4.3.2 Relativistically-broadened iron lines in accreting black holes . . . . . . . . 25

4.4 The fast variability of accreting compact objects . . . . . . . . . . . . . . . 28

4.4.1 Quasi-periodic oscillations in neutron stars . . . . . . . . . . . . . 28

4.4.2 Quasi-periodic oscillations in black holes . . . . . . . . . . . . . 30

5 The Need for a Theoretical Framework for Strong-Field Gravity Tests 32

6 Current Tests of Strong-Field Gravity with Neutron Stars 36

6.1 Brans-Dicke gravity and the orbital decay of binary systems with neutron stars . . 37

6.2 Second-order scalar-tensor gravity and radio pulsars . . . . . . . . . . . . . 38

6.3 Second-order scalar-tensor gravity and X-ray observations of accreting neutron stars 41

7 Going Beyond Einstein $\quad 43$

8 Acknowledgements $\quad 46$

$\begin{array}{lr}\text { References } & 47\end{array}$ 



\section{Introduction}

Over the past 90 years, the basic ingredients of general relativity have been tested in many different ways and in many different settings. From the solar eclipse expedition of 1917 to the modern observations of double neutron stars, general relativity has passed all tests with flying colors [181]. Yet, our inability to devise a renormalizable quantum gravity theory, as well as the mathematical singularities found in many solutions of Einstein's equations, suggest that we should look harder for gravitational phenomena not described by general relativity.

The search for such deviations has been very fruitful in the regime of very weak fields. Observations of high-redshift supernovae $[122,136]$ and of the cosmic microwave background with WMAP [156] have measured a non-zero cosmological constant (or a slowly rolling field that behaves as such at late times). This discovery can be incorporated within the framework of general relativity, if interpreted simply as a constant in the Einstein-Hilbert action. It nevertheless brought to the surface a major problem in trying to connect gravity to basic ideas of quantum vacuum fluctuations $[179,29]$.

In the strong-field regime, on the other hand, which is relevant for the evolution of the very early universe and for determining the properties of black holes and neutron stars, little progress has been made in testing the predictions of general relativity [157]. There are two reasons for this lag. First, phenomena that occur in strong gravitational fields are complex and often explosive, making it very difficult to find observable properties that depend cleanly on the gravitational field and that allow for quantitative tests of gravity theories. Second, there exists no general theoretical framework within which to quantify deviations from general relativistic predictions in the strongfield regime.

During the current decade, technological advances and increased theoretical activity have led to developments that promise to make strong-field gravity tests a routine in the near future. The first generation of earth-based gravitational wave observatories (such as LIGO [88], GEO600 [62], TAMA300 [163], and VIRGO [175]) as well as the Beyond Einstein Missions (such as IXO, LISA, and the Black Hole Imager [16]) will offer an unprecedented look into the near fields of black holes and neutron stars. Moreover, recent ideas on quantum gravity [27], brane-world gravity [90], or other Lagrangian extensions of general relativity $[184,154]$ will provide the means with which the experimental results will be interpreted.

In this article, I review the theoretical and experimental prospects of testing strong-field general relativity with observations in the electromagnetic spectrum. In the first few sections, I discuss the motivation for performing such tests and then describe the astrophysical settings in which strongfield effects can be measured. In Section 5, I elaborate on the need for a theoretical framework within which strong-field gravity tests can be performed and in Section 6 I review the current quantitative tests of general relativity in the strong-field regime that use neutron stars. Finally, in Section 7, I discuss the prospect of probing and testing strong gravitational fields with upcoming experiments and observatories. 


\section{The motivation for strong-field tests}

Most physical scientists would agree that there is very little need to motivate testing one of the fundamental theories of physics in a regime that experiments have probed only marginally, so far. However, in the particular case of testing the strong-field predictions of general relativity, there exist at least three arguments that provide additional strong support to such an endeavor. First, there is no fundamental reason to choose Einstein's equations over other alternatives. Second, gravitational tests to date seldom probe strong gravitational fields. Finally, it is known that general relativity breaks down in the strong-field regime. I will now elaborate on each of these arguments.

- There is no fundamental reason to choose Einstein's equations over other alternatives. - All theories of gravity, including Newton's theory and general relativity, have two distinct ingredients. The first describes how matter moves in the presence of a gravitational field. The second describes how the gravitational field is generated in the presence of matter. For Newtonian dynamics, the first ingredient is Newton's second law together with the assertion that the gravitational and inertial masses of an object are the same; the second ingredient is Poisson's equation. For general relativity, the first ingredient arises from the equivalence principle, whereas the second is Einstein's field equation.

The equivalence principle, in its various formulations, dictates the geometric aspects of the theory [181]: it is impossible to tell the difference between a reference frame at rest and one freely falling in a gravitational field by performing local, non-gravitational (for the Einstein Equivalence Principle) or even gravitational (for the Strong Equivalence Principle) experiments. Moreover, the equivalence principle encompasses the Lorentz symmetry, as well as our belief that there is no preferred frame and position anywhere in the universe. Because of its central importance in any gravity theory, there have been many attempts during the last century at testing the validity of the equivalence principle. These were performed mostly in the weak-field regime and have resulted in upper limits on possible violations of this principle that are as stringent as one part in $10^{12}[181$.

Contrary to the case of the equivalence principle, there are no compelling arguments one can make that lead uniquely to Einstein's field equation. In fact, Einstein reached the field equation, more or less, by reverse engineering (see the informative discussion in [102, 117]) and, soon afterwards, Hilbert constructed a Lagrangian action that leads to the same equation. The EinsteinHilbert action is directly proportional to the Ricci scalar, $R$,

$$
S=\frac{c^{4}}{16 \pi G} \int d^{4} x \sqrt{-g}(R-2 \Lambda)
$$

where $g \equiv \operatorname{det}\left|g_{\mu \nu}\right|, g_{\mu \nu}$ is the spacetime metric, $c$ is the speed of light, $G$ is the gravitational constant, and $\Lambda$ is the cosmological constant. While such a theory is entirely self-consistent at the classical level, it may represent only an approximation that is valid at the scales of curvature that are found in terrestrial, solar, and stellar-system tests.

Indeed, a self-consistent theory of gravity can also be constructed for any other action that obeys the following four simple requirements [102]. It has to: (i) reproduce the Minkowski spacetime in the absence of matter and the cosmological constant, (ii) be constructed from only the Riemann curvature tensor and the metric, (iii) follow the symmetries and conservation laws of the stressenergy tensor of matter, and (iv) reproduce Poisson's equation in the Newtonian limit. Of all the possibilities that meet these requirements, the field equations that are derived from the EinsteinHilbert action are the only ones that are also linear in the Riemann tensor. Albeit simple and elegant, a more general classical action of the form [154]

$$
S=\frac{c^{4}}{16 \pi G} \int d^{4} x \sqrt{-g} f(R)
$$

Living Reviews in Relativity

http: //www. livingreviews.org/lrr-2008-9 
also obeys the same requirements. Indeed, the action (2) results in a field equation that allows for the Minkowski solution in the absence of matter, is constructed only from the Riemann tensor, obeys the usual symmetries and conservation laws [178], and can be made to produce negligible corrections at the small curvatures probed by weak-field gravitational experiments. On the other hand, the predictions of the theory may be significantly different at the strong curvatures probed by gravitational tests involving compact objects.

The single, rank-2 tensor field $g_{\mu \nu}$ (i.e., the metric) of the Einstein-Hilbert action may also not be adequate to describe completely the gravitational force (although, if additional fields are introduced, then the strong equivalence principle is violated, with important implications for the frame and time-dependence of gravitational experiments). In fact, a variant of such theories with an additional scalar field, the Brans-Dicke theory [22], has been the most widely used alternative to general relativity to be tested against experiments. Today, scalar-tensor theories are among the prime candidates for explaining the acceleration of the universe at late times (the "dark energy" [121]). Depending on the coupling between the metric, the scalar field, and matter, the relative contribution of such additional fields may become significant only at the high curvatures found in the early universe or in the vicinity of compact objects.

Although the above discussion has considered only the classical action of the gravitational field in a phenomenological manner, it is also important to note that corrections to the Einstein-Hilbert action occur naturally in quantum gravity theories and in string theory. For example, if we choose to interpret the metric $g_{\mu \nu}$ as a quantum field, we can take Equation (1) as a quantum fieldtheoretic action defined at an ultraviolet scale (such as the Planck scale), and proceed to perform quantum-mechanical calculations in the usual way [50]. However, radiative corrections will induce an infinite series of counter-terms as we flow to lower energies and such counter-terms will not be reabsorbed into the original Lagrangian by adjusting its bare parameters. Instead, such terms will appear as new, higher-derivative correction terms in the Einstein-Hilbert action (1).

Finally, it is worth emphasizing that the previous discussion focuses on Lagrangian gravity in a four-dimensional spacetime. In the context of string theory, general relativity emerges only as a leading approximation. String theory also predicts an infinite set of non-linear terms in the scalar curvature, all suppressed by powers of the Planck scale. Moreover, the low-energy effective action of string theory contains additional scalar (dilatonic) and vector gravitational fields [67]. Motivated by ideas of string theory, brane-world gravity $[90,51,52,53]$ also provides a theory that is consistent with all current tests of gravity.

All the above strongly support the notion that the field equation that arises from the EinsteinHilbert action may be appropriate only at the scales that have been probed by current gravitational tests. But how deep have we looked?

- Gravitational tests to date seldom probe strong gravitational fields. - All historical tests of general relativity have been performed in our solar system. The strongest gravitational field they can, therefore, probe is that at the surface of the Sun, which corresponds to a gravitational redshift of

$$
z_{\odot} \simeq \frac{G M_{\odot}}{R_{\odot} c^{2}} \simeq 2 \times 10^{-6}
$$

and to a spacetime curvature of

$$
\frac{G M_{\odot}}{R_{\odot}^{3} c^{2}} \simeq 4 \times 10^{-28} \mathrm{~cm}^{-2} .
$$

Coincidentally, the gravitational fields that have been probed in tests using double neutron stars are of the same magnitude, since the masses and separation of the two neutron stars in the systems under consideration are comparable to the mass and radius of the Sun, respectively ${ }^{1}$. These are

\footnotetext{
${ }^{1}$ Note, however, that some of the phenomena observed in double neutron stars depend on the coupling of matter and gravity in the strong-field regime [39].
} 
substantially weaker fields than those found in the vicinities of neutron stars and stellar-mass black holes, which correspond to a redshift of $\sim 1$ and a spacetime curvature of $\simeq 2 \times 10^{-13} \mathrm{~cm}^{-2}$.

It is also instructive to compare the degree to which current tests verify the predictions of general relativity to the increase in the strength of the gravitational field going from the solar system to the vicinity of a compact object. Current constraints on the deviation of the PPN parameters from the general relativistic predictions are of order $\simeq 10^{-5}$ [181]. It is conceivable, therefore, that deviations consistent with these constraints can grow and become of order unity when the redshift of the gravitational field probed is increased by six orders of magnitude and the spacetime curvature by fifteen!

Is it possible, however, that general relativity still accurately describes phenomena that occur in the strong gravitational fields found in the vicinity of stellar-mass black holes and neutron stars?

- General relativity breaks down in the strong-field regime. - Our current understanding of the physical world leaves very little doubt that the theory of general relativity itself breaks down at the limit of very strong gravitational fields. Considering the theory simply as a classical, geometric description of the spacetime leads to predictions of infinite matter densities and curvatures in two different settings. Integrating the Oppenheimer-Snyder equations, which describe the collapse of a cloud of dust [112], forward in time leads to the formation of a black hole with a singularity at its center. Integrating the Friedmann equation, which describes the evolution of a uniform and isotropic universe, backward in time always results in a singularity at the beginning of time, the Big Bang. Clearly, the outcome in both of these settings is unphysical.

It is widely believed that quantum gravity prohibits these unphysical situations that occur at the limit of infinitely strong gravitational fields. Even though none of the observable astrophysical objects offer the possibility of testing gravity at the Planck scale, they will nevertheless allow the placing of constraints on deviations from general relativity that are as large as $\sim 10$ orders of magnitude more stringent compared to all other current tests. This is the best result we can expect in the near future to come out of the detection of gravitational waves and the observation of the innermost regions of neutron stars and black holes with NASA's Beyond Einstein missions. If the history of the recent detection of a minute, yet non-zero, cosmological constant is any measure of our inability to predict even the order of magnitude of gravitational effects that we have not directly probed, then we might be up for a pleasant surprise! 


\section{Astrophysical and Cosmological Settings of Strong Grav- itational Fields}

\subsection{When is a gravitational field strong?}

Looking at the Schwarzschild spacetime, it is natural to measure the "strength" of the gravitational field at a distance $r$ away from an object of mass $M$ by the parameter

$$
\epsilon \equiv \frac{G M}{r c^{2}}
$$

which is proportional to the Newtonian gravitational potential and is also directly related to the redshift. Infinitesimal gravitational fields correspond to the limit $\epsilon \rightarrow 0$, which leads to the Minkowski spacetime of special relativity. Weak gravitational fields correspond to $\epsilon \ll 1$, which leads to Newtonian gravity. Finally, the strongest gravitational fields accessible to an observer are characterized by $\epsilon \rightarrow 1$, at which point the black-hole horizon of an object of mass $M$ is approached. (Note that formally the horizon of a Schwarzschild black hole occurs at $\epsilon=2$; I drop here the factor of 2 , as I am mostly interested in dimensional arguments.)

Albeit useful in defining post-Newtonian expansions, the parameter $\epsilon$ is not fundamental in characterizing a gravitational field in Einstein's theory. Indeed, the geodesic equation and the Einstein field equation (or equivalently, the Einstein-Hilbert action [1]) are written in terms of the Ricci scalar, the Ricci tensor, and the Riemann tensor, all of which measure the curvature of the field and not its potential. As a result, when we consider deviations from general relativity that arise by adding terms linearly to the Einstein-Hilbert action, the critical strength of the gravitational field beyond which these additional terms become important is typically given in terms of the spacetime curvature.

For example, in the presence of a cosmological constant, the metric of a spherically-symmetric object becomes

$$
d s^{2}=-\left(1-\frac{2 G M}{r c^{2}}-\frac{\Lambda r^{2}}{3}\right) d t^{2}+\left(1-\frac{2 G M}{r c^{2}}-\frac{\Lambda r^{2}}{3}\right)^{-1} d r^{2}+r^{2}\left(d \theta^{2}+\sin ^{2} \theta d \phi^{2}\right)
$$

and the Newtonian approximation becomes invalid when

$$
\frac{G M}{c^{2} r^{3}} \ll \frac{1}{6} \Lambda
$$

In this case, a gravitational field is "weak" if the spacetime curvature is smaller than $1 / 6 \Lambda$, independent of the value of the parameter $\epsilon$. In the opposite extreme, if there are additional terms in the action of the gravitational field beyond the Einstein-Hilbert term, such as

$$
S=\frac{c^{4}}{16 \pi G} \int d^{4} x \sqrt{-g}\left(R+\alpha R^{2}\right)
$$

then the general relativistic predictions become inaccurate at strong gravitational fields defined by the condition

$$
\frac{G M}{c^{2} r^{3}} \gg \frac{1}{\alpha}
$$

even if the parameter $\epsilon$ is much smaller than unity. Note that, in Equation (8), $\alpha$ is an appropriate constant with units of (length) ${ }^{2}$ and I have set the Ricci scalar to $R \sim G M / r^{3} c^{2}$ (I use this here as an order of magnitude estimate and do not consider the fact that, if the distance $r$ is larger than the radius of the object, then the Ricci scalar in general relativity vanishes). 
Similar considerations lead to a condition on curvature when we add to the Einstein-Hilbert action terms that invoke additional scalar, vector, and tensor fields. In all these cases, a strong gravitational field is characterized not by a large gravitational potential (i.e., a high value of the parameter $\epsilon$ ) but rather by a large curvature

$$
\xi \equiv \frac{G M}{r^{3} c^{2}}
$$

Because the condition that the curvature needs to satisfy in order for a gravitational field to be considered "strong" depends on the particular deviation from general relativity under study (cf. Equations [7] and [9]) I will not normalize the parameter $\xi$ to any particular energy density but rather leave it, hereafter, as a dimensional quantity.

This is an appropriate parameter with which to measure the strength of a gravitational field in a geometric theory of gravity, such as general relativity, because the curvature is the lowest order quantity of the gravitational field that cannot be set to zero by a coordinate transformation. Moreover, because the curvature measures energy density, a limit on curvature will correspond to an energy scale beyond which additional gravitational degrees of freedom may become important.

\subsection{A parameter space for tests of gravity}

The two parameters, $\epsilon$ and $\xi$, define a parameter space on which we can quantify the strengths of the gravitational fields probed by different tests of gravity (see Figure 1). Only a fraction of this parameter space is accessible to experiments. Regions of the parameter space with potential $\epsilon>1$ correspond to distances from a gravitating object that are smaller than the horizon radius and are, therefore, inaccessible to observers. (I neglect here, for simplicity, the small numerical factor in the horizon radius that depends on the spin of the black hole.) In Figure 1, this region is outlined by the vertical red line.

Quantifying deviations from general relativity for part of the parameter space requires a detailed understanding of the properties of dark matter and dark energy, which is beyond current capabilities. In the limit of very small values of the curvature, the presence of a non-zero cosmological constant affects the outcome of gravitational experiments when (see Equation [7])

$$
\xi \leq \frac{3 G \Omega_{\Lambda} H_{0}^{2}}{8 \pi c^{2}} \simeq 5 \times 10^{-58}\left(\frac{\Omega_{\Lambda}}{0.73}\right)\left(\frac{H_{0}}{73 \mathrm{~km} / \mathrm{s} / \mathrm{Mpc}}\right)^{2} \mathrm{~cm}^{-2}
$$

where $\Omega_{\Lambda}$ is the current density of dark energy in units of the critical density and $H_{0}$ is the current value of the Hubble constant. Phenomena that probe such low values of curvature (i.e., below the horizontal green line in Figure 1) can lead to quantitative tests of general relativity only if a specific model of dark energy (e.g., a cosmological constant) is assumed.

The ability to perform a quantitative test of a gravity theory also relies on an independent measurement of the mass that generates the gravitational field. This is not always possible, especially in various cosmological settings, where gravitational phenomena are used mostly to infer the presence of dark matter and not to test general relativistic predictions. Dark matter is typically required in systems for which the acceleration drops below the so-called MOND acceleration scale $a_{0} \simeq 10^{-8} \mathrm{~cm} \mathrm{~s}^{-2}[97,140,14]$. (This is an observed fact, independent of whether the inability of Newtonian gravity to account for observations is due to the presence of dark matter or to the breakdown of the theory itself.) This acceleration scale is also comparable to $a_{0} \simeq c H_{0}$. Systems for which dark matter is necessary to account for their gravitational fields are characterized by

$$
\xi \leq\left(\frac{a_{0}}{c^{2}}\right)^{2} \frac{1}{\epsilon} \simeq\left(\frac{H_{0}}{c}\right)^{2} \frac{1}{\epsilon} .
$$

Living Reviews in Relativity

http: //www. livingreviews.org/lrr-2008-9 


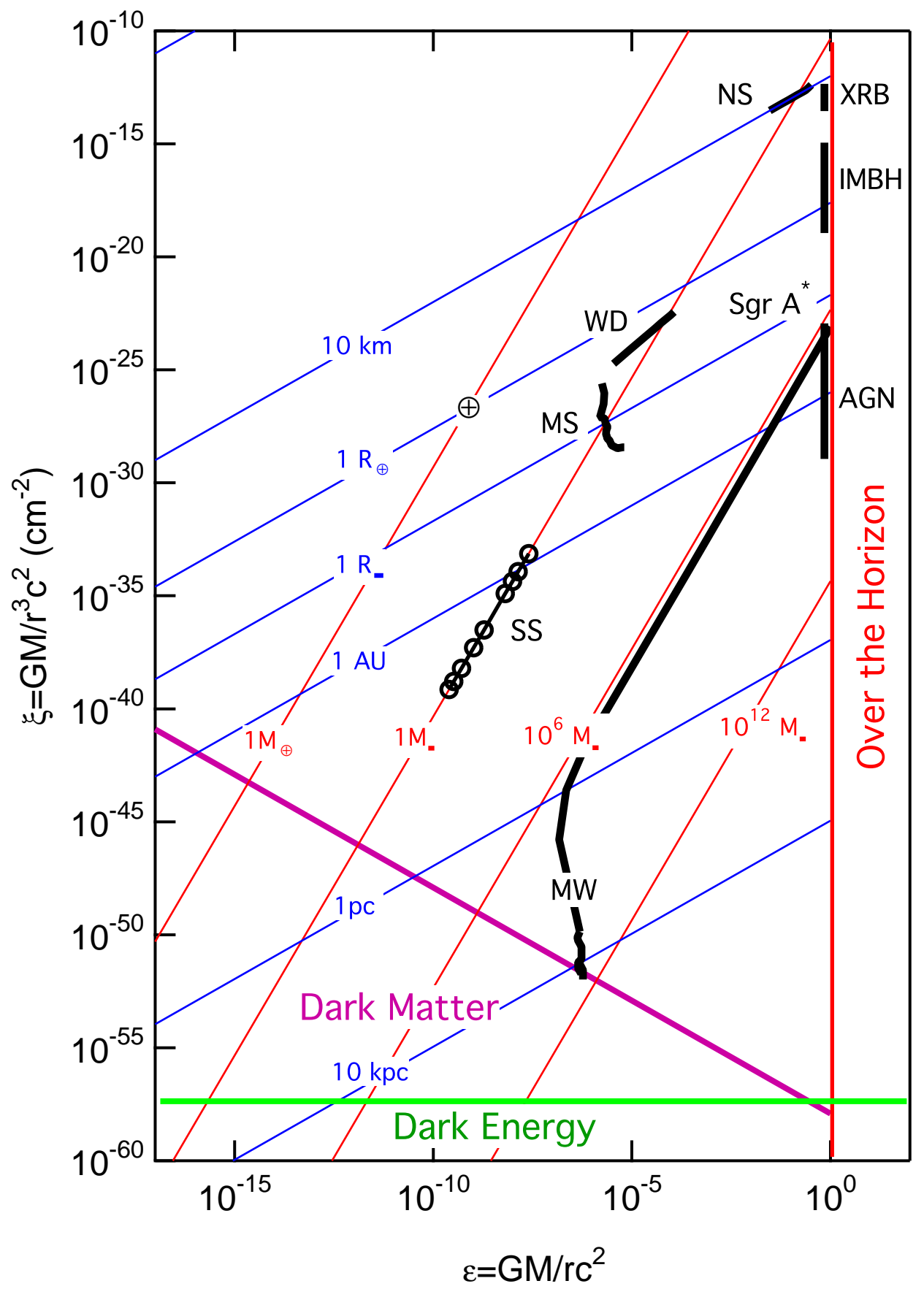

Figure 1: A parameter space for quantifying the strength of a gravitational field. The $x$-axis measures the potential $\epsilon \equiv G M / r c^{2}$ and the $y$-axis measures the spacetime curvature $\xi \equiv G M / r^{3} c^{2}$ of the gravitational field at a radius $r$ away from a central object of mass $M$. These two parameters provide two different quantitative measures of the strength of the gravitational fields. The various curves, points, and legends are described in the text. 
This region of the parameter space is outlined by the purple line in Figure 1 . The fact that the three lines that correspond to the Schwarzschild horizon, the MOND acceleration scale, and the dark energy all seem to intersect roughly in one point in the parameter space is directly related to the cosmic-coincidence problem, i.e., that the universe is flat, with comparable amounts of (mostly dark) matter and dark energy.

In the opposite limit of very strong gravitational fields, general relativity is expected to break down when quantum effects become impossible to neglect. This is expected to happen if a gravitational test probes a distance from an object of mass $M$ that is comparable to the Compton wavelength $\lambda_{\mathrm{C}} \equiv h / M c$, where $h$ is Planck's constant. Quantum effects are, therefore, expected to dominate when

$$
\xi \geq \frac{1}{L_{\mathrm{P}}^{2}} \epsilon^{2}
$$

where $L_{\mathrm{P}} \equiv\left(G h / c^{3}\right)^{1 / 2} \simeq 4 \times 10^{-33} \mathrm{~cm}$ is the Planck length. This part of the parameter space is not shown in Figure 1, as it is many orders of magnitude away from the values of the parameters that correspond to astrophysical systems.

Having defined the parameter space and outlined the various limiting cases, I can now identify the astrophysical systems that probe its various regimes. In general, systems of constant central mass $M$ will follow curves of the form

$$
\xi=\frac{c^{4}}{G^{2} M^{2}} \epsilon^{3}
$$

whereas probes at a constant distance $r$ away from the central object will follow curves of the form

$$
\xi=\frac{1}{r^{2}} \epsilon .
$$

Figure 1 shows a number of representative contours of constant mass and distance.

The strongest gravitational fields around astrophysical systems can be found in the vicinities of neutron stars (NS in Figure 1) and black holes in X-ray binaries (XRB). Large gravitational potentials but smaller curvatures can be found around the horizons of intermediate-mass black holes $\left(\sim 10^{2}-10^{4} M_{\odot} ; \mathrm{IMBHs}\right)$ and in active galactic nuclei $\left(10^{6}-10^{10} M_{\odot} ; \mathrm{AGN}\right)$. Weaker gravitational fields exist near the surfaces of white dwarfs (WD), main-sequence stars (MS), or at the distances of the various planets in our solar system (SS). Finally, even weaker gravitational fields are probed by observations of the motions of stars in the vicinity of the black hole in the center of the Milky Way (Sgr A*), and by studies of the rotational curve of the Milky Way (MW) and other galaxies. In placing the various systems on the parameter space shown in Figure 1, I have used a typical mass-radius relation for neutron stars and white dwarfs [147], the calculated mass-radius relation of main-sequence stars [34], and the inferred mass-radius profile of the inner region around Sgr A* [143], which smoothly approaches the mass profile inferred from the rotation curve of the Milky Way [46].

Current tests of general relativity with astrophysical objects probe a wide range of gravitational potentials and curvatures (see Figure 2). However, they fall short of probing the most extreme phenomena that are predicted by the theory to occur in the vicinities of compact objects, for example, tests during solar eclipses, with double neutron stars (such as the Hulse-Taylor pulsar), or with Grav Prob B probe curvatures that are the same as those found near the horizons of supermassive black holes, but potentials that are smaller by six to ten orders of magnitude. Moreover, all these tests probe curvatures that are smaller by thirteen or more orders of magnitude from those found near the surfaces of neutron stars and the horizons of stellar-mass black holes. Future experiments, such as the gravitational wave detectors and the Beyond Einstein missions, will offer for the first time the opportunity to probe directly such strong gravitational fields.

Living Reviews in Relativity

http: //www . livingreviews . org/lrr-2008-9 


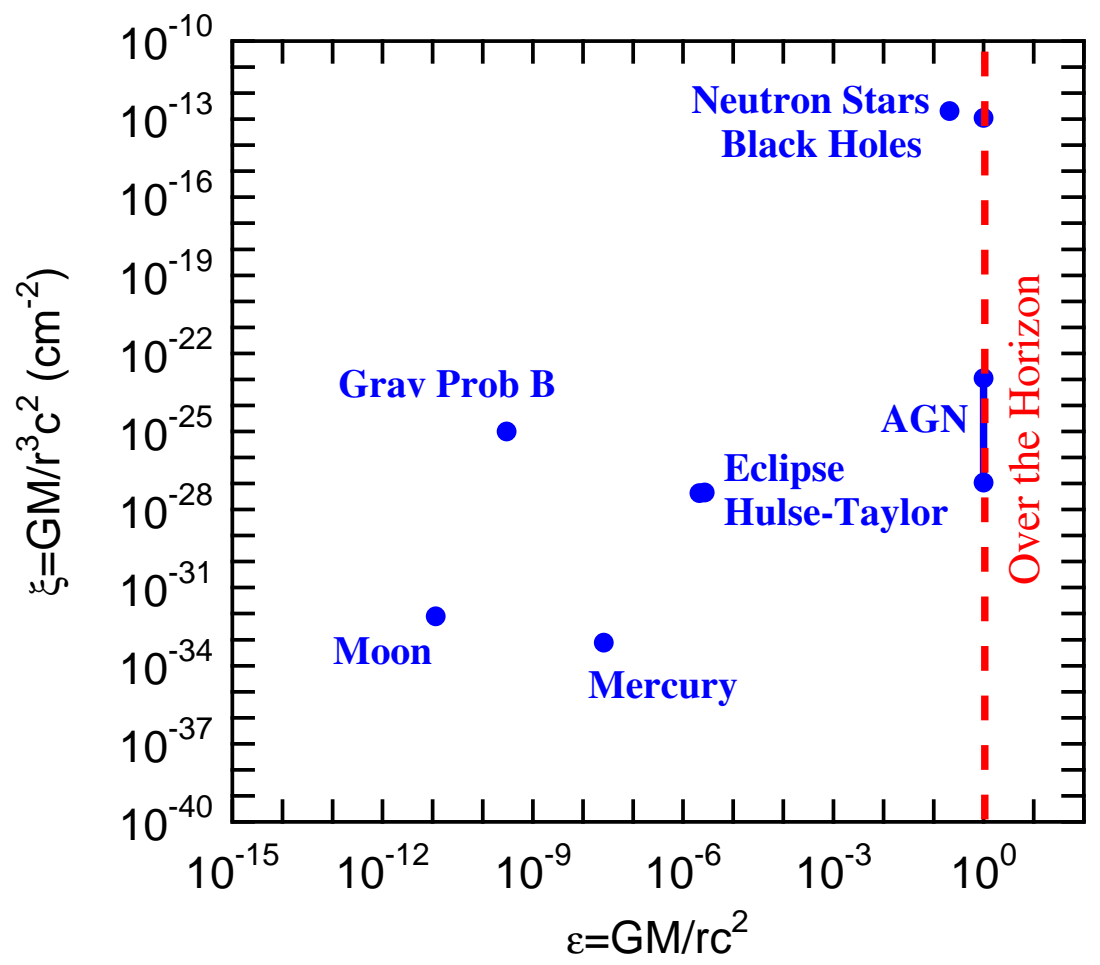

Figure 2: Tests of general relativity placed on an appropriate parameter space. The long-dashed line represents the event horizon of Schwarzschild black holes. 
The whole range of gravitational fields, from the weakest to the strongest, can also be found during various epochs of the evolution of the universe. As a result, observations of cosmological phenomena may also probe very strong gravitational fields. The scalar curvature of a flat universe is given by

$$
R=\frac{6}{\alpha^{2}}\left(\alpha \ddot{\alpha}+\dot{\alpha}^{2}\right)
$$

where $\alpha$ is the scale factor. Using the Friedmann equation, the scalar curvature becomes

$$
R=3\left(\frac{\Omega_{\mathrm{m}}^{0}}{\alpha^{3}}+4 \Omega_{\mathrm{d}}^{0}\right)\left(\frac{H_{0}}{c}\right)^{2},
$$

where $\Omega_{\mathrm{m}}^{0}$ and $\Omega_{\mathrm{d}}^{0}$ are the (non-relativistic) matter and dark energy densities in the present universe, respectively, in units of the critical density. Equation (17) shows that, at late times, the radius of curvature of the universe is comparable to the Hubble distance.

The evolution of the scalar curvature with redshift for a flat universe and for the best-fit cosmological parameters obtained by the WMAP mission [156] is shown in Figure 3. Identified on this figure are several characteristic epochs that have been used in testing general relativistic predictions: the $z \simeq 1$ epoch of type I supernovae that are used to measure the value of the cosmological constant $[122,136]$, the $z \simeq 1000$ epoch at which the acoustic peaks of the cosmic microwave background observed by WMAP are produced, and the period of nucleosynthesis during which the temperature of the universe was in the range $60 \mathrm{keV}-1 \mathrm{MeV}[141,31]$. The period of BigBang nucleosynthesis is the earliest epoch for which quantitative tests have been performed. The corresponding scalar curvature of the universe at that time, however, is still small and comparable to the curvatures of gravitational fields probed by current tests of general relativity in the solar system. It was only when the temperature of the universe was $\sim 100 \mathrm{GeV}$ that its curvature was $\simeq 10^{-12} \mathrm{~cm}^{-2}$, i.e., comparable to that found around a neutron star or stellar-mass black hole. This is the period of electroweak baryogenesis, for which no detailed theoretical models or data exist to date.

\subsection{Probing versus testing strong-field gravity}

The parameter space shown in Figure 1 is useful in identifying the strength of the gravitational field probed by a particular test of gravity. However, it is important to emphasize that probing a gravitational field of a given strength is not necessarily the same as testing general relativity in that regime. I discuss bellow the difference with two examples from scalar-tensor gravity that illustrate the two opposite extremes.

First, a phenomenon that occurs in a weak gravitational field may actually be testing the strong-field regime of gravity. In general relativity, Birkhoff's theorem states that the external spacetime of a spherically-symmetric object is described by the Schwarzschild metric, independent of the properties of the object itself. Birkhoff's theorem, however, does not apply to a variety of gravity theories, such as scalar-tensor or non-linear (e.g., $R+R^{2}$ ) theories. In fact, in these theories, the spacetime at any point around a spherically-symmetric object depends on the mass distribution that generates the spacetime, which may itself lie in a strong gravitational field and, therefore, probe that regime of the theory. For example, in Brans-Dicke gravity, which is a special case of scalar-tensor theories, the evolution of the binary orbit in a system with two neutron stars due to the emission of gravitational waves depends on the coupling of matter to the scalar field, which occurs in the strong gravitational field of each neutron star [54, 182, 40]. As a result, even though the gravitational field that corresponds to a double-neutron star orbit is rather weak (see Figure 2), observations of the orbital decay of the binary actually test general relativity against scalar-tensor theories in the strong-field regime [40].

Living Reviews in Relativity

http: //www. livingreviews.org/lrr-2008-9 


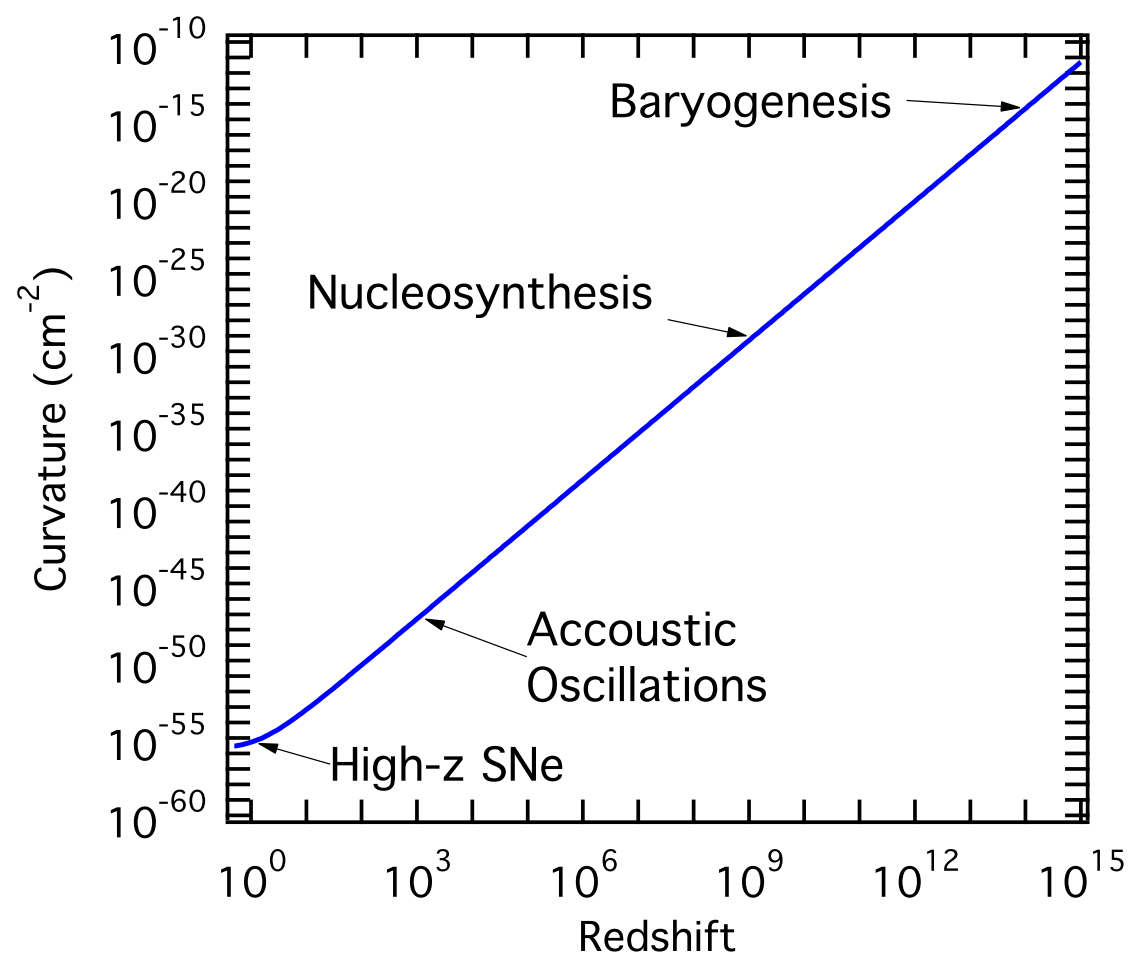

Figure 3: The scalar curvature of our universe, as a function of redshift. The curve corresponds to a flat universe with the best-fit values of the cosmological parameters obtained by the WMAP mission [156]. The arrows point to the curvature and redshift of the universe during various epochs. 
In the opposite extreme, phenomena that probe strong gravitational fields may not necessarily be used in testing general relativity in this regime. Analytical and numerical studies strongly suggest that the end state of the collapse of a star in Brans-Dicke gravity is a black hole described by the Kerr spacetime of general relativity [165, 13, 71, 142, 130]. Therefore, the observation of a phenomenon that occurs even just above the horizon of a black hole cannot be used in testing general relativity against Brans-Dicke gravity in the strong-field regime, because both theories make the exact same prediction for that phenomenon.

In the following, I will distinguish attempts to probe phenomena that occur exclusively in the strong-field regime of general relativity from those that aim to test the strong-field predictions of the theory against various alternatives. 


\section{Probing Strong Gravitational Fields with Astrophysical Objects}

A number of astrophysical objects offer the possibility of detecting directly the observable consequences of two strong-field predictions of general relativity that have no weak-field or Newtonian counterparts: the presence of a horizon around a collapsed object and the lack of stable circular orbits in the vicinity of a neutron star or black hole. As in most other areas of astrophysics research, we have to rely on imaging, spectral, or timing observations in order to reveal the information of the strong-field effects that is encoded in the detected photons. The construction of gravitational wave observatories will offer, for the first time in the near future, a wealth of additional probes into the inner workings of gravitational fields in the vicinities of compact objects.

In the following, I review a number of recent attempts to probe strong-field phenomena that have used a variety of techniques and were applied to different astrophysical objects. I will only discuss phenomena that are observable in the electromagnetic spectrum and refer to a number of excellent reviews on the gravitational phenomena that are anticipated to be detected by gravitational wave observatories $[137,58]$.

\subsection{Black-hole images}

To paraphrase the common proverb, a picture is worth a thousand spectra. Directly imaging the vicinity of a black hole promises to provide direct evidence for the existence of a horizon. However, black holes are notoriously small, and the resolution required for imaging their horizons is, for most cases, beyond current capabilities. For a stellar-mass black hole in the galaxy, the opening angle of the horizon, as viewed by an observer on Earth, is only

$$
\theta=2 \times 10^{-4}\left(\frac{M}{10 M_{\odot}}\right)\left(\frac{1 \mathrm{kpc}}{D}\right) \mu \operatorname{arcsec},
$$

where $M$ is the mass of the black hole and $D$ is its distance.

For a supermassive black hole in a distant galaxy, the opening angle is

$$
\theta=20\left(\frac{M}{10^{9} M_{\odot}}\right)\left(\frac{1 \mathrm{Mpc}}{D}\right) \mu \operatorname{arcsec} .
$$

This is shown in Figure 4 for a number of supermassive black holes with secure mass determinations. The angular size of the horizons of some of the sources are barely resolvable today with interferometric observations in the sub-mm/infrared wavelengths and will be resolvable in the X-ray wavelengths in the near future with the Black Hole Imager [114].

The black hole that combines the highest brightness with the largest angular size of the horizon is the one that powers the source Sgr $A^{*}$, in the center of the Milky Way. Since the first measurements of the size of the source at $7 \mathrm{~mm}$ [89] and at $1.4 \mathrm{~mm}$ [79] demonstrated that the emitting region is only a few times larger than the radius of the horizon (see Figure 5), a number of observational and theoretical investigations have aimed to probe deeper into the gravitational field of the black hole and constrain its properties.

The long-wavelength spectrum of Sgr A* peaks at a frequency of $\simeq 10^{12} \mathrm{~Hz}$, suggesting that the emission changes from optically thick (probably synchrotron emission) to optically thin at a comparable frequency (see, e.g., [107]). As a result, observations at frequencies comparable to or higher than the transition frequency can, in principle, probe the accretion flow at regions very close to the horizon of the black hole.

Even though the exact shape and size of the image of Sgr A* at long wavelengths depends on the detailed structure of the underlying accretion flow (cf. [116] and [186]), there exist two generic 


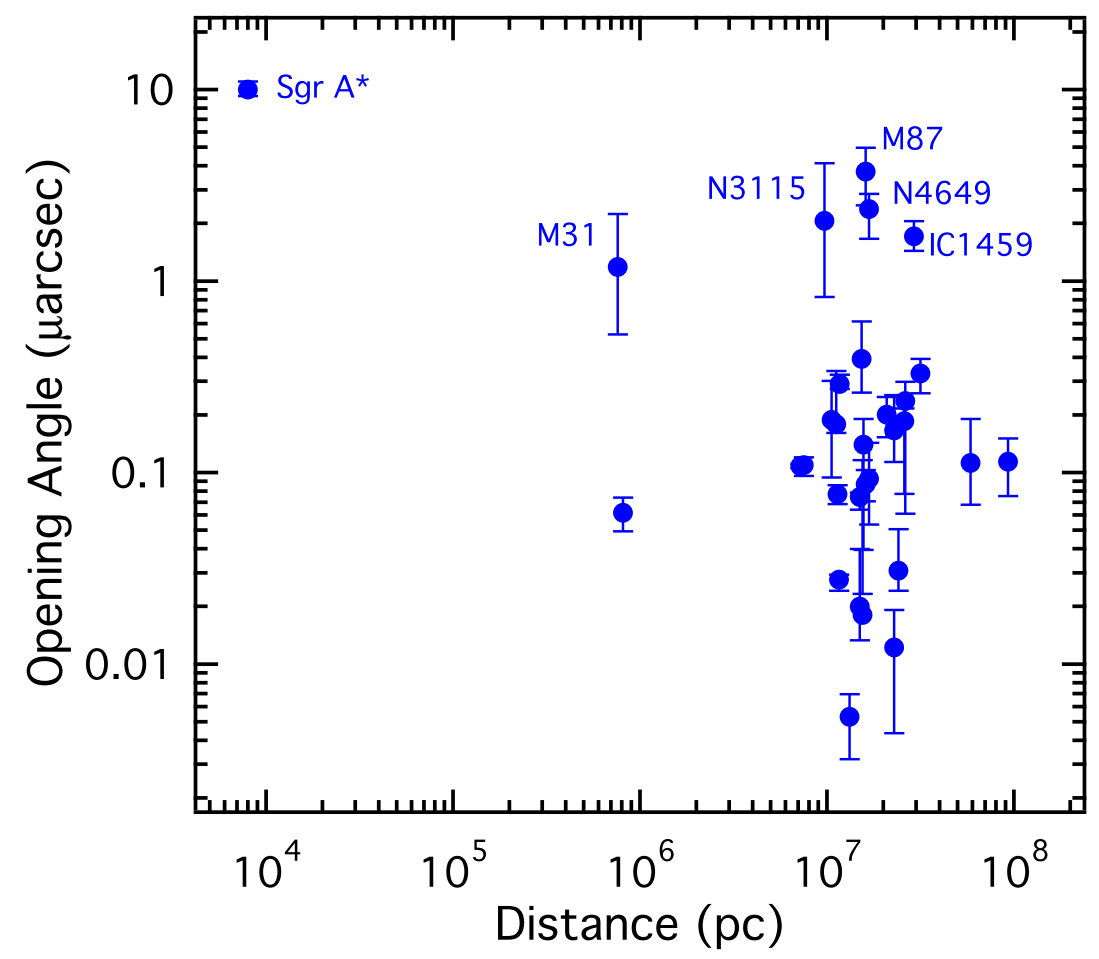

Figure 4: The opening angles, as viewed by an observer on Earth, of the horizons of a number of supermassive black holes in distant galaxies with a secure dynamical mass measurement (sample of [169]). The opening angle of the black-hole horizon in the center of the Milky Way ( $\mathrm{Sgr} \mathrm{A}^{*}$ ) is also shown for comparison. 


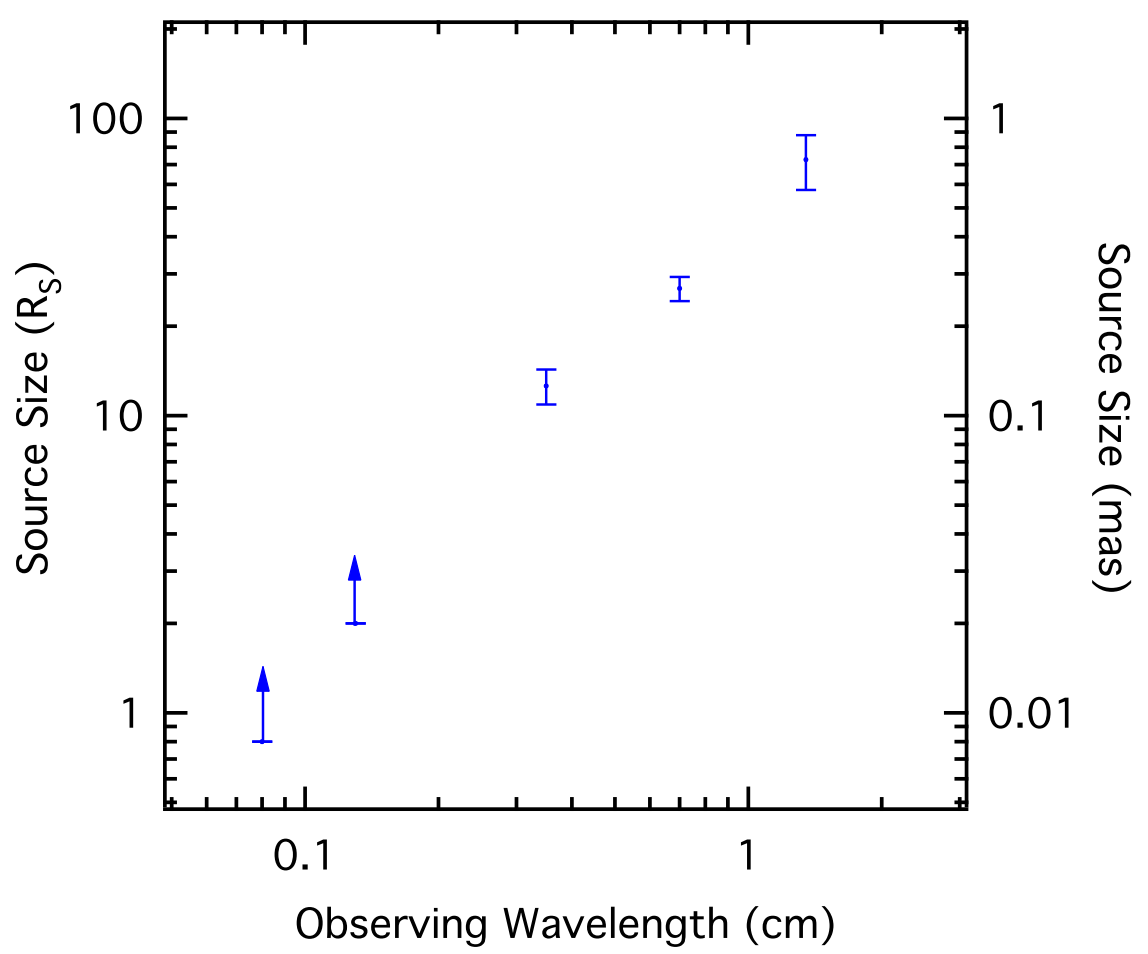

Figure 5: The major axis of the accretion flow around the black hole in the center of the Milky Way, as measured at different wavelengths, in units of the Schwarzschild radius (left axis) and in milliarcsec (right axis; adapted from [148]). Even with current technology, the innermost radii of the accretion flow can be readily observed. 
observable signatures of its strong gravitational field. First, the horizon leaves a 'shadow' on the image of the source, which is equal to $\simeq \sqrt{27} G M / c^{2}$ and roughly independent of the spin of the black hole $[7,57,162,24,110]$. Second, the brightness of the image of the accretion flow is highly non-uniform because of the high velocity of the accreting plasma and the effects of the strong gravitational lensing. Simultaneously fitting the size, shape, polarization map, and centroid of the image observed at different wavelengths with future telescopes, will offer the unique possibility of removing the complications introduced by the unknown nature of the accretion flow, imaging directly the black-hole shadow, and measuring the spin of the black hole [25].

\subsection{Continuum spectroscopy of accreting black holes}

There have been at least three different efforts published in the literature that use the luminosities and the continuum spectra of accreting black holes to look for evidence of strong-field phenomena.

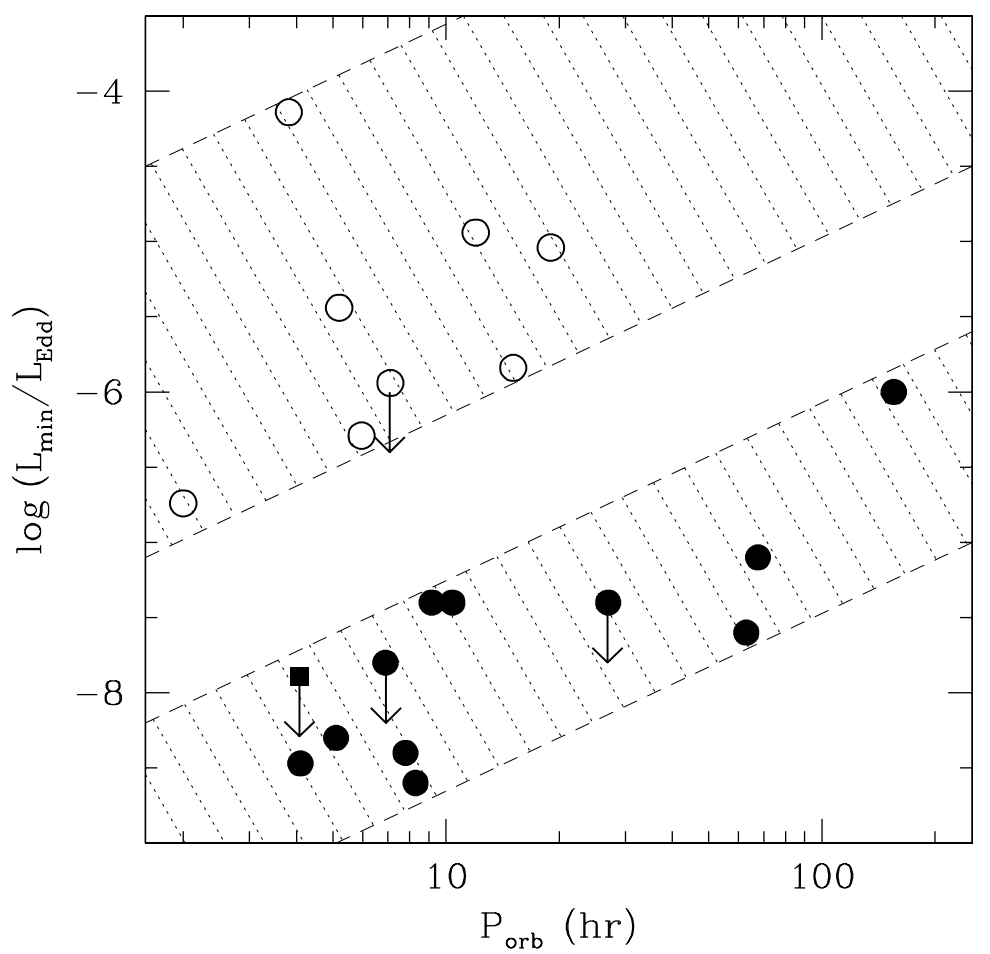

Figure 6: The $2-20 \mathrm{keV}$ quiescent luminosities of black-hole candidates (filled circles) and neutron stars (open circles) in units of the Eddington luminosity for different galactic binary systems, as a function of their orbital periods, which are thought to determine the mass transfer rate between the two stars. The systematically lower luminosities of the black-hole systems have been attributed to the presence of the event horizon [106, 91].

\subsubsection{Luminosities of black holes in quiescence and the absence of a hard surface}

Low-mass X-ray binaries are stellar systems in which the primary star is a compact object and the secondary star is filling its Roche lobe. Matter is transferred from the companion star to the compact object and releases its gravitational potential energy mostly as high-energy radiation, making these systems the brightest sources in the X-ray sky [126, 92]. 
The rate with which mass is transfered from the companion star to the compact object is determined by the ratio of masses of the two stars, the evolutionary state of the companion star, and the orbital separation [173]. On the other hand, the rate with which energy is released in the form of high-energy radiation depends on the rate of mass transfer, on the state of the accretion flow (i.e., whether it is via a geometrically thin disk or a geometrically thick but radiatively inefficient flow), and on whether the compact object has a hard surface or an event horizon. Indeed, for a neutron-star system in steady state, most of the released gravitational potential energy has to be radiated away (only a small fraction heats the stellar core [26]), whereas for a black-hole system, a significant amount of the potential energy may be advected inwards past the event horizon, and hence may be forever lost from the observable universe. For similar systems, in the same accretion state, one would therefore expect black holes to be systematically less luminous than neutron stars [106].

The luminosities of transient black holes and neutron stars in their quiescent states most clearly show this trend. When plotted against the orbital periods of the binary systems, which are used here as observable proxies to the mass transfer rates, sources that are believed to be black holes, based on their large masses, are systematically less luminous (Figure 6 and [106, 61, 91]). Although the physical mechanism behind the difference in luminosities is still a matter of debate [106, 18, 82], the trend shown in Figure 6 appears to be a strong, albeit indirect, evidence for the presence of an event horizon in compact objects with masses larger than the highest possible mass of a neutron star.

\subsubsection{Hard X-ray spectra of luminous black holes and the presence of an event hori- zon}

Galactic black holes in some of their most luminous states (the so-called very high states) have mostly thermal spectra in the soft X-rays with power-law tails that extend well into the soft $\gamma$-rays [68]. It has been hypothesized that these power-law tails are the result of Compton upscattering of soft X-ray photons off the relativistic electrons that flow into the black-hole event horizon with speeds that approach the speed of light and, therefore, constitute an observational signature of the presence of an event horizon (e.g., see $[168,84])$.

A relativistic converging flow has indeed the potential of producing power-law spectral tails (e.g., see $[120,167,123])$. However, this mechanism is identical to a second-order Fermi acceleration and hence the power-law tail is a result of multiple scatterings away from the horizon with low energy exchange per scattering rather than the result of very few scatterings of photons with ultrarelativistic electrons near the black-hole horizon [128, 118]. Moreover, the model spectra always cut off at energies lower than the electron rest mass [84, 109], whereas the observed spectra extend into the MeV range [68]. Successful theoretical models of the power-law spectra of black holes that are based on Comptonization of soft photons by non-thermal electrons [65], as well as the discovery of similar power-law tails in the spectra of accreting neutron stars that extend to $\sim 100-200 \mathrm{keV}[48,47]$, have shown conclusively that the observed power-law tails do not constitute evidence of black-hole event horizons.

\subsubsection{Measuring the radii of the innermost stable circular orbits of black holes using continuum spectra}

The thermal spectrum of a black-hole source in some of its most luminous states is believed to originate in a geometrically thin accretion disk. The temperature profile of such an accretion disk away from the black hole is determined entirely by energy conservation and is independent of the magnitude and properties of the mechanism that transports angular momentum and allows for matter to accrete (as long as this mechanism is local; see $[146,5]$ ). The situation is very different, however, near the radius of the innermost stable circular orbit (hereafter ISCO). 
Inside the ISCO, fluid elements cannot stay in circular orbits but instead quickly loose centrifugal support and rapidly fall into the black hole. The density of the accretion disk inside the ISCO is very small and the viscous heating is believed to be strongly diminished. It is, therefore, expected that only material outside the ISCO contributes to the observed thermal spectrum. The temperature profile of the accretion flow just outside the ISCO depends rather strongly on the mechanism that transports angular momentum outwards and in particular on the magnitude of the torque at the ISCO $[80,59,3]$. To lowest order, however, if the entire accretion disk spectrum can be decomposed into a sum of black bodies, each at the local temperature of the radial annulus in which it originates, then the highest temperature will be that of the plasma near the ISCO and the corresponding flux of radiation will be directly proportional to the square of the ISCO radius.

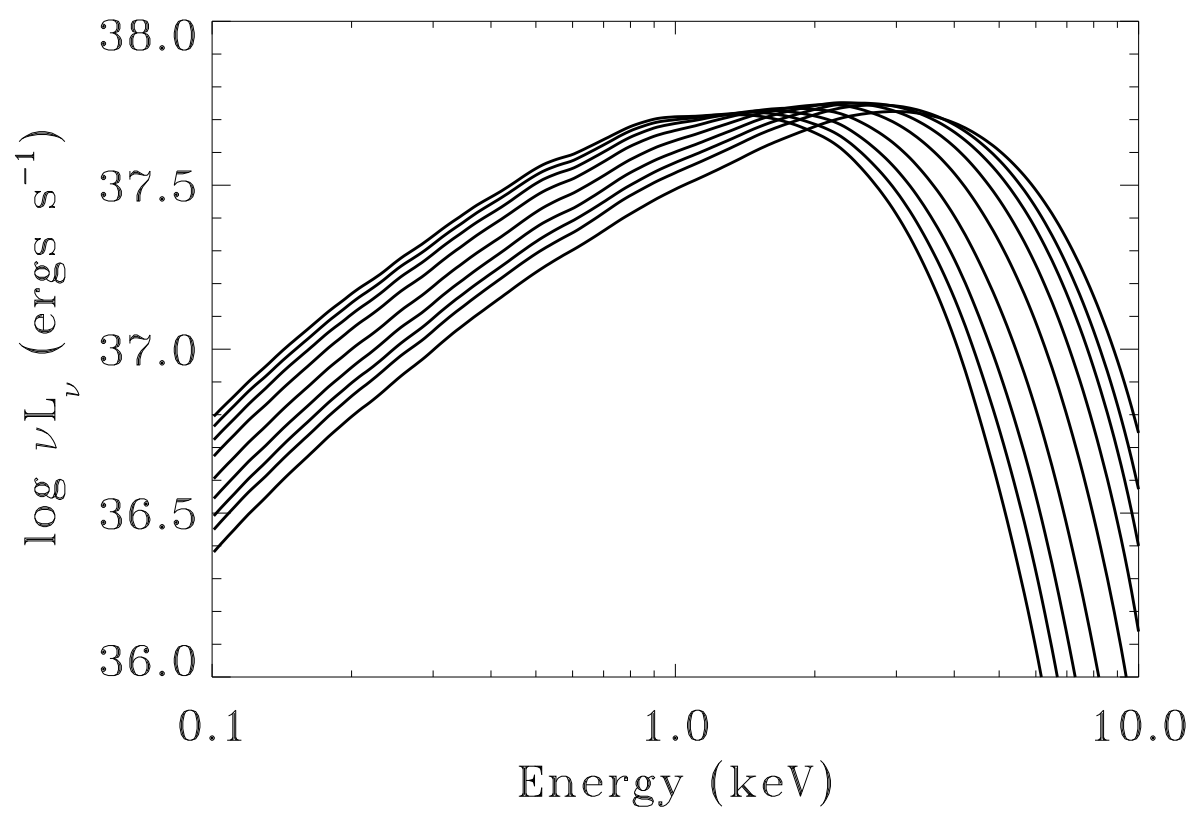

Figure 7: The spectra emerging from geometrically thin accretion disks around black holes with different spins, but with the same accretion luminosity [41].From left to right, the curves correspond to spins $(a / M)$ of $0,0.2,0.4,0.6,0.78,0.881,0.936,0.966$, and 0.99 . The spin values were chosen to give roughly equal variation in the position of the spectral peak for spins $>0.8$. The other parameters that determine the model are the viscosity parameter, $\alpha=0.01$, the inclination of the observer, $\cos i=0.5$, the mass of the black hole, $M=10 M_{\odot}$, and the accretion luminosity, $L=0.1 L_{\mathrm{Edd}}$. The peak energies of the spectra increase with increasing spin, as a consequence of the fact that the ISCO radius decreases with spin.

Phenomenological fits of multi-temperature black-body models to the observed spectra of black holes provide strong support to the above interpretation. When model spectra are fit to observations of any given black hole in luminosity states that differ by more than one order of magnitude, the inferred ISCO radius remains approximately constant [86]. For systems with a dynamically measured mass and with a known distance, such an observation can lead to a measurement of the physical size of the ISCO and hence of the spin of the black hole (see Figure 7) [187, 64].

There are a number of complications associated with producing the model spectra of multitemperature black-body disks that are required in measuring spectroscopically the ISCO radius around a black hole. First, as discussed above, the temperature profile of an accretion disk at 
the region around the ISCO depends very strongly on the details of the mechanism of angular momentum transport, which are poorly understood [80, 59, 3]. Second, the vertical structure of the disk at each annulus, which determines the emerging spectrum, may or may not be in hydrostatic equilibrium near the ISCO, as it is often assumed, and its structure depends strongly on the external irradiation of the disk plasma by photons that originate in other parts of the disk. Finally, material in the inner accretion disk is highly ionized and often far from local thermodynamic equilibrium, generating spectra that can be significantly different from black bodies [72].

There have been a number of approximate models of multi-temperature accretion disks that take into account some of these effects, in a phenomenological or ab initio way. The models of Li et al. [87], based on the alpha model for angular momentum transport, assume that the local emission from each annulus is a black body at the local temperature, and take into account the strong lensing of the emitted photons by the central black hole. On the other hand, the models of Davis et al. [41] are the result of ionization-equilibrium and radiative-transfer calculations at each annulus; they are based on the alpha model for angular momentum but allow for non-zero torques at the ISCO, and take into account the strong lensing of photons by the black hole.

Given the flux $F$ of the accretion disk measured by an observer on Earth, the color temperature $T_{\text {col }}$ that corresponds to the innermost region in the disk that is emitting (which presumably is near the ISCO), the distance $D$ to the source, and the mass $M$ of the black hole, the spin $\alpha$ of the black hole can be inferred [187] by equating the radius of the ISCO, i.e.,

$$
r_{\mathrm{ISCO}}=\sqrt{\frac{2 G M}{c^{2}}}\left\{3+A_{2} \pm\left[\left(3-A_{1}\right)\left(3+A_{1}+2 A_{2}\right)\right]^{1 / 2}\right\},
$$

to the one inferred spectroscopically (since $F \sim T^{4} R^{2}$ ) by

$$
r_{\mathrm{spec}}=D\left[\frac{F}{2 \sigma g(\theta, \alpha)}\right]^{1 / 2}\left[\frac{f_{\mathrm{col}} f_{\mathrm{GR}}(\theta, \alpha)}{T_{\mathrm{col}}}\right]^{2} .
$$

Here $A_{1}=1+\left(1-a^{2}\right)^{1 / 3}\left[(1+a)^{1 / 3}+(1-a)^{1 / 3}\right], A_{2}=\left(3 a^{2}+A_{1}^{2}\right)^{1 / 2}, a$ is the specific angular momentum per unit mass for the black hole, and the positive (negative) sign is taken for prograde (retrograde) disks. In these equations, $\sigma$ is the Stefan-Boltzmann constant and $\theta$ is the inclination of the observer with respect to the symmetry axis of the accretion disk. The functions $g(\theta, \alpha)$ and $f_{\mathrm{GR}}(\theta, \alpha)$ are correction factors for the flux and the temperature, respectively, that need to be calculated when going from an accretion disk annulus to a distant observer and incorporate the combined effects of gravitational lensing, gravitational redshift, and Doppler boosting of the disk photons. Given a thickness of the accretion disk, both these transfer functions can be computed to any desired degree of accuracy. Finally, the factor $f_{\text {col }}$ measures the ratio of the color temperature of the spectrum (as measured by fitting a black body to the observed spectrum) to the effective temperature in that annulus in the accretion disk (which is a measure of the total radiation flux emerging from that annulus). Computing the value of the factor $f_{\text {col }}$ is the goal of the recent calculations of the ionization equilibrium and radiative transfer in accretion disks [41].

Fitting these spectral models to a number of observations of black-hole candidates with dynamically measured masses has resulted in approximate measurements of their spins: $a>0.7$ for GRS $1915+105[96,93] ; a=0.75-0.85$ for $4 \mathrm{U} 1543-47$ [145]; $a=0.65-0.75$ for GRO J165540 [145]. It is remarkable that all inferred values of the black-hole spins are high, comparable to the maximum allowed by the Kerr solution.

Equations (20) and (21) demonstrate the strong dependence of the inferred values of blackhole spins on various observable quantities (the mass of, distance to, and inclination of the black hole, as well as the flux, and temperature of its disk spectrum) and on a model parameter (the color correction factor $f_{\text {col }}$ ). Numerical simulations of magnetohydrodynamic flows onto black holes are finely tuned to resolve the length and timescales of phenomena that occur in the vicinity 
of the horizon of a black hole (see, e.g., [60, 42]). When such models incorporate accurate multidimensional radiative transfer, they will provide the best theoretical spectra to be compared directly to observations (see, e.g., [20]). Moreover, monitoring of the same sources at long wavelengths will improve the measurements of their masses and distances. Finally, combination of this with other methods based on line spectra and the rapid variability properties of accreting black holes will enable us to tighten the uncertainties in the various model parameters and observed quantities that enter Equation (20) and measure with high precision the spins of galactic black holes.

\subsection{Line spectroscopy of accreting compact objects}

Heavy elements on the surface layers of neutron stars or in the accretion flows around black holes that are not fully ionized generate atomic emission and absorption lines that can be detected by a distant observer after suffering a large gravitational redshift. The value of the gravitational redshift can be used to uniquely identify the region in the spacetime of the compact object in which the observed photons are produced.

\subsubsection{Atomic lines from the surfaces of neutron stars}

The gravitational redshift of an atomic line from the surface layer of a neutron star leads to a unique determination of the relation between its mass and radius. The detection of a rotationallybroadened atomic line from a rapidly spinning neutron star offers the additional possibility of measuring directly the stellar radius [115, 32] and, therefore, of determining its mass, as well. The profile of a rotationally-broadened atomic line can be used to study frame-dragging effects in the strong-field regime [17]. Moreover, detecting a gravitationally-redshifted and rotationallybroadened atomic line can lead to a measurement of the oblateness of the spinning star [28], which is determined by the strong-field coupling of matter with the gravitationally field. Unfortunately, this is one of the very few astrophysical settings discussed in this review in which observations significantly trail behind theoretical investigations.

Despite many optimistic expectations and early claims (see, e.g., [85]), the observed spectra of almost all weakly-magnetic neutron stars are remarkably featureless. The best studied case is that of the nearby isolated neutron star RX J1856-3754, which was observed for $450 \mathrm{ks}$ with the Chandra X-ray Observatory and showed no evidence for any atomic lines from heavy elements [21]. This is, in fact, not surprising, given that heavy elements drift into the photosphere in timescales of minutes [19] and it takes only $\simeq 10^{-7} M_{\odot}$ of light elements to blanket a heavy element surface.

There are two types of neutron stars, however, in the atmospheres of which heavy elements may abound: young cooling neutron stars and accreting X-ray bursters [115]. On the one hand, the escaping latent heat of the supernova explosion makes young neutron stars relatively bright sources of X-rays. Their strong magnetic fields can inhibit the accretion of light elements either from the supernova fallback or from the interstellar medium, leaving the surface heavy elements exposed. On the other hand, in the atmospheres of accreting, weakly-magnetic neutron stars, heavy elements are continuously replenished. Moreover, large radiation fluxes pass through their atmospheres during thermonuclear bursts [161] making them very bright and easily detectable.

The most promising detection to date of gravitationally-redshifted lines from the surface of a neutron star came from an observation with XMM-Newton of the source EXO 0748-676, which showed redshifted atomic lines during thermonuclear flashes [37]. This is a slowly spinning neutron star (47 Hz [174]) and hence its external spacetime can be accurately described by the Schwarzschild metric. In this case, the measurement of a gravitational redshift of $z=0.35$ leads to a unique determination of the relation between the mass and the radius of the neutron star, i.e., $M \simeq$ $1.4(R / 10 \mathrm{~km}) M_{\odot}$. The combination of this result with the spectral properties of thermonuclear bursts during periods of photospheric radius expansion and in the cooling tails also allowed for an independent determination of the mass and radius of the neutron star [113].

Living Reviews in Relativity

http: //www. livingreviews.org/lrr-2008-9 
Future observations of bursting or young neutron stars with upcoming X-ray missions such as IXO [74] and XEUS [185] have the potential to detect many gravitationally-redshifted atomic lines and, hence, to probe the coupling of matter to the strong gravitational fields found in the interiors of neutron stars.

\subsubsection{Relativistically-broadened iron lines in accreting black holes}

Astrophysical black holes in active galactic nuclei accreting at moderate rates offer another possibility for probing strong gravitational fields using atomic spectroscopy (for an extensive review on the subject see [133]; see also [99] for a review of iron line observations from stellar-mass black holes). The relatively cool accretion disks in these systems act as large mirrors, reflecting the high-energy radiation that is believed to be produced in the disk coronae by magnetic flaring [69]. The spectrum of reflected radiation in hard X-rays is determined by electron scattering, whereas the spectrum in the soft X-rays is characterized by a large number of fluorescent lines caused by bound-bound transitions of the partially ionized material. The combination of the high yield and relatively high abundance of iron atoms in the accreting material make the iron $\mathrm{K} \alpha$ line, with a rest energy of $6.4 \mathrm{keV}$ for a neutral atom, the most prominent feature of the spectrum.
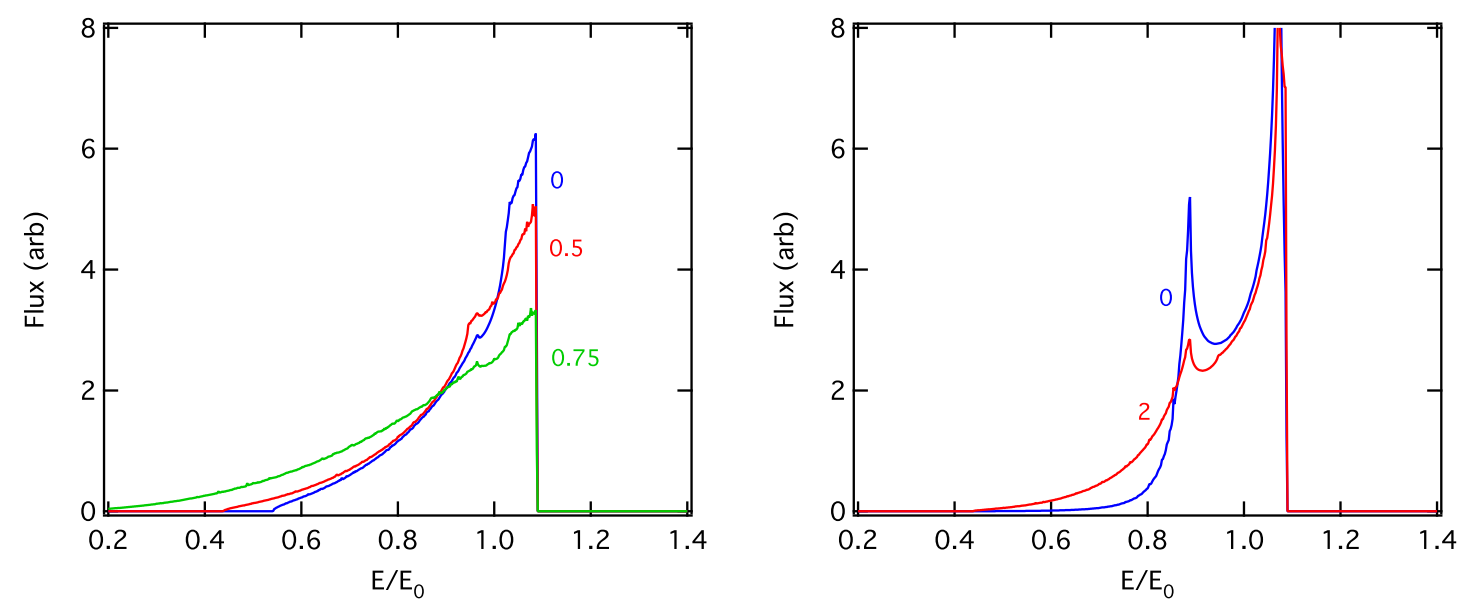

Figure 8: Theoretical models of relativistically-broadened iron line profiles from accretion flows around black holes. The left panel shows the dependence of the line profile on the spin parameter of the black hole, whereas the right panel shows its dependence on the emissivity index (see text). All calculations were performed for an inclination angle of $40^{\circ}$ [23].

The profile of the fluorescent iron line as observed at infinity is determined mainly by general and special relativistic effects that influence the propagation of photons from the point of reflection to the observer [81]. Dividing an accretion disk into a series of concentric rings orbiting at the local Keplerian frequency, special relativistic effects produce a rotational splitting of the line emerging from each ring, whereas general relativistic effects generate an overall redshift [55]. The combination of these effects integrated over the entire surface of the accretion disk leads to a characteristic profile for the iron reflection line, which is broad with a shallow and extended red wing (Figure 8).

The magnitude of the relativistic effects depends on the specifics of the spacetime of the black hole, the position and orientation of the observer, the position and properties of the source of Xrays above the accretion disk, and the dependence of fluorescence yield on position of the accretion disk through its dependence on the ionization states of the elements [63]. Given a model for the source of X-rays and the accretion disk, fitting the profile of an iron line from an accreting black 
hole can lead, in principle, to a direct mapping of its spacetime. Unfortunately, the source of X-ray illumination and the physical properties of the accretion flows themselves are poorly understood.

If we make assumptions regarding these astrophysical complications that are largely model independent, a general property of the spacetime, such as the spin of the black hole, can be measured. The accretion disk is typically modeled as a geometrically thin reflecting surface at the rotational equator of the black hole that extends inwards to the radius of the innermost stable circular orbit. Even though the density of the material inside this radius is significant and might reflect the illuminating X-rays, its ionization state changes rapidly, leading to small changes in the resulting iron line profile $[132,23]$. The extent of the iron line towards lower energies is a measure of the innermost radius of the accretion disk. By assumption, this radius is set as the radius of the innermost stable circular orbit, which depends on the spin of the black hole. Fitting theoretical models to observations can, therefore, lead to a measurement of the black-hole spin.

The uncertainties in the position of the illuminating source and in the disk structure are often modeled by a single function for the "emissivity" of the iron line, which measures the flux in the iron line that emerges locally from each patch on the accretion disk. This is typically taken to be axisymmetric and to have a power-law dependence on radius, i.e., $r^{-a}$. Increasing the emissivity index $a$ results in iron-line profiles with more extended red wings, which is degenerate with increasing spin of the black hole (see Figure 8 and [12]). This uncertainty can introduce significant systematic errors in modeling iron-line profiles from slowly-spinning black holes. For rapidly-spinning black holes, however, masking the effect of the black-hole spin by steepening the emissivity function requires an unphysically high value for the emissivity index [23].

Since the original observation of broadened iron lines from the supermasive black hole MCG6-15-30 with ASCA [164], observations of other active galactic nuclei with ASCA [104], XMMNewton [105], and more recently with Suzaku [131], as well as of stellar-mass black holes [98], have revealed many more examples of such redshifted atomic lines. The best studied case remains MCG-6-15-30 (see Figure 9), in which the extended red wing of the line has been discussed as evidence for a rapidly-spinning black hole $(\alpha \geq 0.98$ [23]).

Perhaps the most challenging, although most rewarding to understand, property of iron lines is their time variability. Current observations of iron lines from accreting black holes (e.g., the one shown in Figure 9) are integrated over a time that is equal to many hundred times the dynamical timescale in the accretion-disk region, where the lines are formed. As a result, an observed line profile is not the result of reflection from an accretion disk of a single flaring event, but rather the convolution of many such events that occurred over the duration of the observation. Moreover, the continuum spectrum of the black hole, which is presumably reflected off the accretion disk to produce the fluorescent iron line, changes over longer timescales, implying a correlated variability of the line itself.

Observations with current instruments can only investigate the correlated variability of the iron line with the continuum spectrum (see, however, [73]). They have shown that the flux in the line remains remarkably constant, even though the continuum flux changes by almost an order of magnitude [56]. General relativistic light bending, which leads to focusing of the photon rays towards the innermost regions of the accretion disk, may be responsible for this puzzling effect [101].

Future observations with upcoming X-ray missions, such as IXO [74] and XEUS [185], will resolve the time evolution of the reflected iron line from a single magnetic flare [134]. Because density inhomogeneities in the turbulent accretion flow move, roughly, in test-particle orbits [4], the time evolution of the redshift of the iron line from a single flare reflected mainly off a localized density inhomogeneity will allow for a direct mapping of the spacetime around the black hole.

Living Reviews in Relativity

http: //www. livingreviews.org/Irr-2008-9 

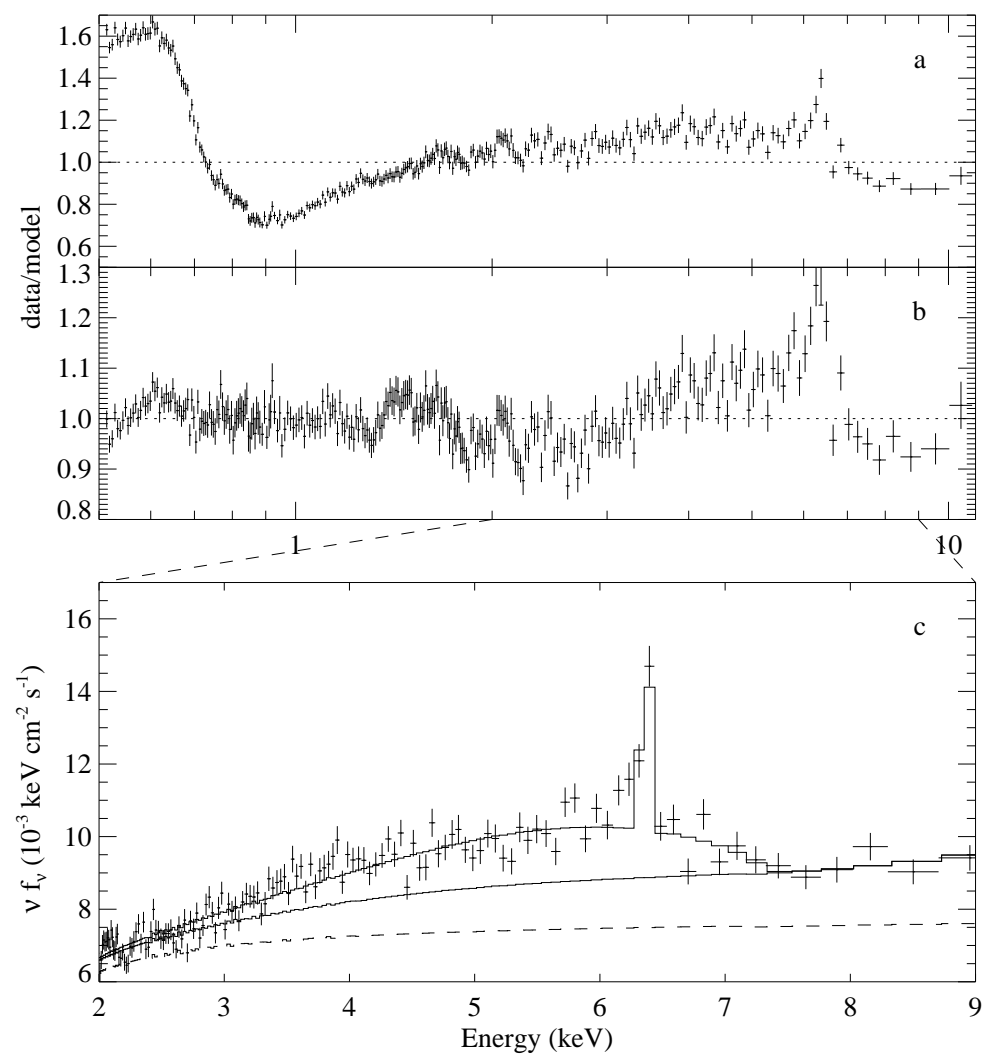

Figure 9: The $0.5-10 \mathrm{keV}$ spectrum of the supermassive black hole in the center of the galaxy MCG-615-30 as observed with XMM-EPIC. Panel (a) shows the ratio of the observed spectrum to a power-law model and reveals the complicated structure of the residuals. Panel (b) shows the ratio of the observed spectrum to a model of the warm absorber, which accounts for the low-energy residuals. Panel (c) shows the 2-9 keV spectrum of the source together with a model of the relativistically-broadened iron line [183]. 


\subsection{The fast variability of accreting compact objects}

The strongest gravitational fields in astrophysics can be probed only with rapidly variable phenomena around neutron stars and galactic black holes (see Figure 18 in Section 7). Such phenomena have been discovered in almost all known accreting compact objects in the galaxy. They are quasiperiodic oscillations (QPOs) with frequencies in the range of $\sim 1 \mathrm{~Hz}-1 \mathrm{kHz}$ that remain coherent for tens to hundreds of cycles and follow a rich and often complicated phenomenology (for an extensive review of the observations see [171]).

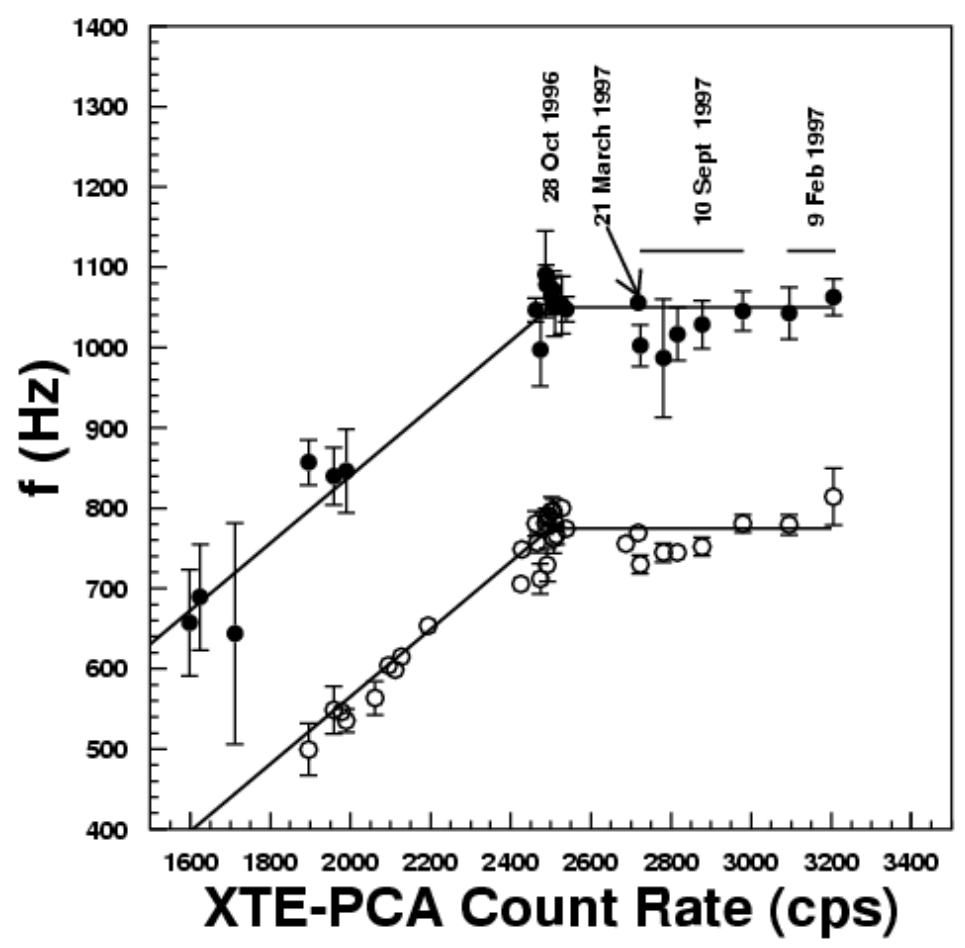

Figure 10: The dependence of the twin QPO frequencies on the X-ray count rate observed by the PCA instrument onboard RXTE, for the neutron-star source 4U 1820-30 [188]. The flattening of the correlation at high frequencies has been discussed as a signature of the innermost stable circular orbit.

\subsubsection{Quasi-periodic oscillations in neutron stars}

The fastest oscillations detected from accreting, weakly-magnetic neutron stars are pairs of QPOs with variable frequencies that reach up to $\sim 1300 \mathrm{~Hz}$ and with frequency separations on the order of $\sim 300 \mathrm{~Hz}$ [171]. The origin of these oscillations is still a matter of debate. However, all current models associate at least one of the oscillation frequencies with a characteristic dynamical frequency in a geometrically thin accretion disk (see discussion in [124, 100, 159, 129]).

The highest dynamical frequency of a mode excited at any radius in an equatorial accretion disk around a compact object is the one associated with the circular orbit of a test particle at that radius [8]; this is often referred to as the azimuthal, orbital, or Keplerian frequency. A mode in the accretion disk associated with this frequency can give rise to a long-lived quasi-periodic oscillation only if it lives outside the innermost stable circular orbit. The azimuthal frequency at this radius provides, therefore, an upper limit on the frequency of any observed oscillation [77, 100].

Living Reviews in Relativity

http: //www. livingreviews.org/lrr-2008-9 
As a result, detecting such rapid oscillations offers the possibility of measuring the location of and understanding the properties of the region near the innermost stable circular orbit around a neutron star.

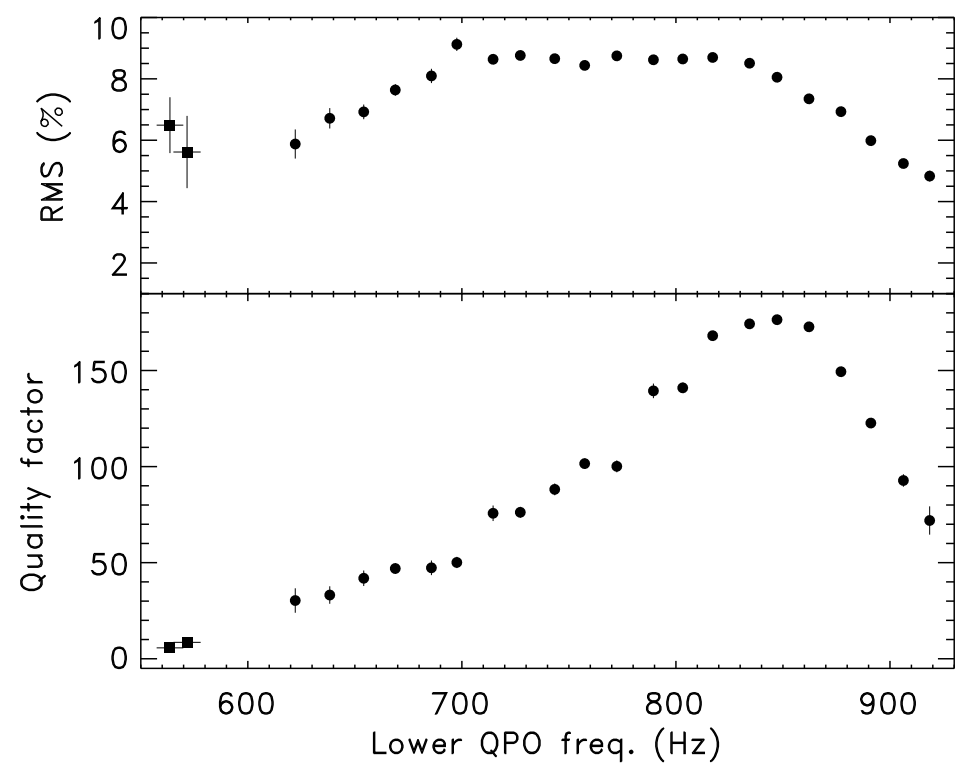

Figure 11: The dependence of the amplitude and quality factor of the lower $\mathrm{kHz} \mathrm{QPO}$ on its frequency for the neutron-star source $4 \mathrm{U}$ 1636-56 [10]. The drop of the QPO amplitude and coherence at high frequencies have been discussed as signatures of the innermost stable circular orbit.

The signature of the ISCO on the amplitudes and characteristics of the observed oscillations is hard to predict without a firm model for the generation of the oscillations in the X-ray flux. Two potential signatures have been discussed, however, based on the phenomenology of the oscillations. The first one is associated with the fact that the frequencies of the oscillations appear to increase roughly with accretion rate. When an oscillation frequency reaches that of the innermost stable circular orbit, one would expect its frequency to remain constant over a wide range of accretion rates [100]. Such a trend has been observed in the quasi-periodic oscillations of the globular cluster source 4U 1820-30 (See [188, 75] and Figure 10). When observations of the source obtained over different epochs are combined, the dependence of the frequency of the fastest oscillation on the observed accretion rate appears to flatten at a value of $\simeq 1050 \mathrm{~Hz}$. This is comparable to the azimuthal frequency at the innermost stable circular orbit for a $\simeq 2.1 M_{\odot}$ neutron star [188].

Albeit suggestive, the interpretation of the $4 \mathrm{U}$ 1820-30 data relies on the assumption that the oscillatory frequencies in an accretion disk depend monotonically on the accretion rate and, furthermore, that the X-ray count rate is a good measure of the accretion rate. This assumption is probably justified for short timescales (of order one day) but is known to break down on longer timescales, such as those used in Figure 10 [170]. Indeed, in a given source, the same oscillation frequencies have been observed over a wide range of X-ray count rates and vice versa [170]. The hard X-ray color of a source, and not the count rate, appears to be a more unique measure of the accretion rate, which is presumably the physical parameter that determines the oscillation frequencies [95]. When the data of $4 \mathrm{U}$ 1820-30 are plotted against hard color, the characteristic flattening seen in Figure 10 disappears [95].

A second signature of the innermost stable circular orbit is a potential decrease in the amplitude and coherence of the oscillations when the region in which they are excited approaches the ISCO. 
Such a trend has been observed in a number of accreting neutron stars (Figure 11 and [10, 11]) and has been questioned on similar grounds as the study of $4 \mathrm{U}$ 1820-30 [94]. The most significant criticism comes from the fact that the drop in amplitude and coherence is rather gradual and occurs over $\mathrm{a} \sim 150 \mathrm{~Hz}$ range of frequencies. Even assuming that this drop is a signature of the ISCO, measuring its location will be possible only within a detailed model of the frequencies of quasi-periodic oscillations.

Among more model-dependent ideas, perhaps the most exciting prospect of probing strong-field gravity effects in neutron stars with quasi-periodic oscillations comes from applying the relativistic model of QPOs [159] to the observed correlations between various pairs of QPO frequencies [127]. In the relativistic model, the highest-frequency QPO is identified with the azimuthal frequency of a test particle in orbit at a given radius. The peak separation of this QPO from the second-higher frequency QPO is identified as the radial epicyclic frequency of the test particle in the same orbit. A variant of this model can account for the observed correlations between oscillation frequencies, when hydrodynamic effects are taken into account [129]. Because the two observed frequencies are directly related to the azimuthal and radial frequencies at various radii in the accretion flow, interpretation of the data with this model can provide a direct map of the exterior spacetime of the neutron stars, to within the $\simeq 10 \%$ uncertainty introduced by the hydrodynamic corrections to the oscillation frequencies.

\subsubsection{Quasi-periodic oscillations in black holes}

Pairs of rapid quasi-periodic oscillations have also been detected from a number of accreting systems that harbor black-hole candidates [92]. The phenomenology of these oscillations is very different from the one discussed above for accreting neutron stars. The frequencies of the rapid oscillations observed in each source vary at most by a percent over a wide range of luminosities and their ratios are practically equal to ratios of small integers (2:3 for XTE J1550-564 and GRO J1655-40, 3:5 for GRS $1915+105$, etc.).

The high frequencies of the oscillations observed from black-hole sources with dynamicallymeasured masses demonstrate that they originate in regions very close to the black-hole horizons. In fact, requiring the frequency of the $450 \mathrm{~Hz}$ oscillation observed from GRO J1655-40 to be limited by the azimuthal frequency at the ISCO necessitates a spinning black hole with a Kerr spin parameter $a / M \geq 0.25$ [160]. Moreover, the frequencies of the observed oscillations are roughly inversely proportional to the black-hole masses, as one would expect if they were associated to dynamical frequencies near the innermost stable circular orbit [2].

As in the case of neutron stars, using black-hole quasi-periodic oscillations to probe directly strong gravitational fields is hampered by the lack of a firm understanding of the physical mechanism that is producing them $[178,1,135,103]$. In one interpretation, they are associated with linear oscillatory modes that are trapped just outside the radius of the innermost stable circular orbit (for reviews see $[176,76,111]$ ). The frequencies of these modes depend primarily on the mass and spin of the black hole. Identifying the two observed oscillations with the lowest-order linear modes, therefore, leads to two pairs of values for the mass and spin of the black hole (depending on which oscillation is identified with which mode). For example, for the case of the black hole GRO J1655-40, one of the inferred pairs of values agrees with the dynamically measured mass of the black hole of $6.9 \pm 1.0 M_{\odot}$ and results in an estimated value of the black-hole spin of $a / M \sim 0.9$ (Figure 12 and [177]). Although compelling, this interpretation leaves to coincidence the fact that the ratios of the oscillation frequencies are approximately equal to ratios of small integers.

In an alternate model, the oscillations are assumed to be excited in regions of the accretion disks where two of the dynamical frequencies are in parametric resonance, i.e., their ratios are equal to ratios of small integers [1]. In this case, the frequencies of the oscillations depend on the mass and spin of the black hole, as well as on the radius at which the resonance occurs. As a result,

Living Reviews in Relativity

http: //www. livingreviews.org/lrr-2008-9 

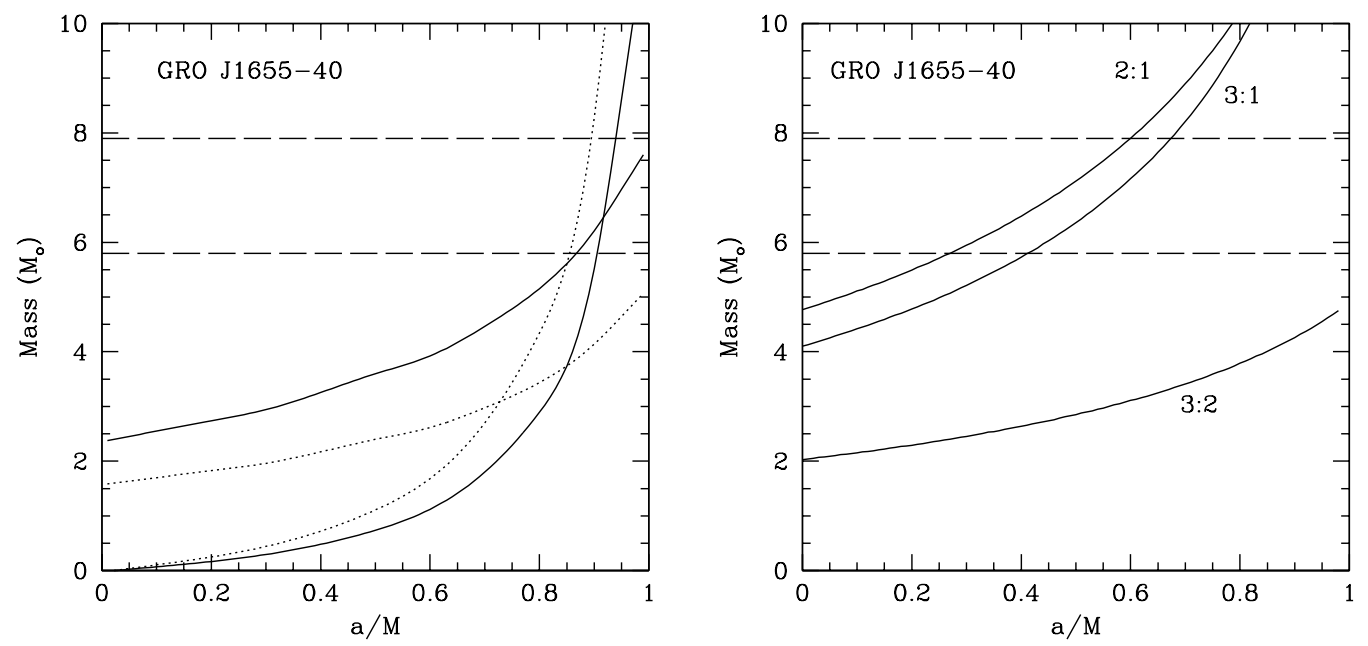

Figure 12: (Left Panel) The intersection of the two solid lines shows the black-hole mass and spin for the source GRO J1655-40 for which the observed $300 \mathrm{~Hz}$ and $450 \mathrm{~Hz}$ oscillations can be explained as the lowest-order $c$ and $g$-modes, respectively. The intersection of the dotted lines makes the opposite identification of disk modes to the observed oscillatory frequencies (after [177]). (Right Panel) Each solid line traces pairs of black-hole mass and spin for which the observed frequencies correspond to different resonances between the Keplerian and periastron precession frequencies (after [1]). In both panels, the horizontal dashed lines show the uncertainty in the dynamically-measured mass of the black hole.

the observation of two oscillations from any given source does not lead to a unique measurement of its mass and spin, but rather to a family of solutions. For example, identifying the frequencies of the two oscillations observed from GRO J1655-40 as a 3:2, a 3:1, or a 2:1 resonance between the Keplerian and the periastron precession frequencies at any radius in the accretion disk leads to three families of solutions, as shown in Figure 12. The dynamically-measured mass of the black hole then picks only two of the possible families of solutions and leads to a smaller value for the inferred spin.

Future observations of accreting neutron stars and black holes with upcoming missions that will have fast timing capabilities, such as XEUS [185], will be able to discover a large spectrum of quasiperiodic oscillations from each source. Such observations will constrain significantly the underlying physical model for these oscillations, which remains the most important source of uncertainty in using fast variability phenomena in probing strong gravitational fields. 


\section{The Need for a Theoretical Framework for Strong-Field Gravity Tests}

Modern observations of black holes and neutron stars in the galaxy provide ample opportunity for testing the predictions of general relativity in the strong-field regime, as discussed in the previous section. In several cases, astrophysical complications make such studies strongly dependent on model assumptions. This will be remedied in the near future, with the anticipated advances in the observational techniques and in the theoretical modeling of the various astrophysical phenomena. A second difficult hurdle, however, in performing quantitative tests of gravity with compact objects will be the lack of a parametric extension to general relativity, i.e., the equivalent of the PPN formalism, that is suitable for calculations in the strong-field regime.

In the past, bona fide tests of strong-field general relativity have been performed using particular parametric extensions to the Einstein-Hilbert action. This appears, a priori, to be a reasonable approach for a number of reasons. First, deriving the parametric field equations from a Lagrangian action ensures that fundamental symmetries and conservation laws are obeyed. Second, the parametric Lagrangian action can be used over the entire range of field strengths available to an observer and, therefore, even tests of general relativity in the weak-field limit (i.e., with the PPN formalism) can be translated into constraints on the parameters of the action. This is often important when strong-field tests lead to degenerate constraints between different parameters. Finally, phenomenological Lagrangian extensions can be motivated by ideas of quantum gravity and string theory and, potentially, help constrain the fundamental scales of such theories. There are, however, several issues that need to be settled before any such parametric extension of the Einstein-Hilbert action can become a useful theoretical framework for strong-field gravity tests (see also [154] and references therein).

First, gravity is highly non-linear and strong-field phenomena often show a non-perturbative dependence on small changes to the theory. I will illustrate this with scalar-tensor theories that result from adding a minimally coupled scalar field to the Ricci curvature in the action. Such fields have been studied for more than 40 years in the form of Brans-Dicke gravity [180] and have been recently invoked as alternatives to a cosmological constant for modeling the acceleration of the universe [121]. In the context of compact-object astrophysics, constraints on the relative contribution of scalar fields coupled in different ways to the metric have been obtained from observations of the orbital decay of double neutron stars [182, 39] and compact X-ray binaries [182, 125]. More recently, similar constraints on scalar extensions to general relativity have been placed using the observation of redshifted lines from an X-ray burster [43] and of quasi-periodic oscillations observed in accreting neutron stars [44]. The oscillatory modes of neutron stars in such theories and the prospect of constraining them using gravitational-wave signatures have also been studied [152, 153].

The general form of the Lagrangian of a scalar-tensor theory is given, in the appropriate frame, by the Bergmann-Wagoner action (see [180] for details)

$$
S=\frac{1}{16 \pi} \int d^{4} x \sqrt{-g_{*}}\left[R_{*} \pm g_{*}^{\mu \nu} \partial_{\mu} \phi \partial_{\nu} \phi+2 \lambda(\phi)\right]+S_{\mathrm{m}}\left[\phi_{m}, A^{2}(\phi) g_{* \mu \nu}\right],
$$

where $A(\phi)$ and $\lambda(\phi)$ are two arbitrary functions, and $S_{m}$ is the action for the matter field $\phi_{m}$. In the strong-field regime, the potential term $\lambda(\phi)$ in the action (22) is typically negligible and is set to zero. On the other hand, the functional form of the coupling function $A(\phi)$ can be parameterized to measure deviations from general relativity.

Damour and Esposito-Farèse [39] considered a second-order parametric form

$$
A(\phi)=\exp \left[\alpha_{0}\left(\phi-\phi_{0}\right)+\frac{1}{2} \beta_{0}\left(\phi-\phi_{0}\right)^{2}+\ldots\right] \text {, }
$$

Living Reviews in Relativity

http: //www. livingreviews . org/lrr-2008-9 
with $\phi_{0} \rightarrow 0$ a background cosmological value for the scalar field and $\alpha_{0}$ and $\beta_{0}$ the two parameters of the theory to be constrained by observations. The linear term, parameterized by $\alpha_{0}$, can be best constrained with weak-field tests. On the other hand, constraining significantly the non-linear term, parameterized by $\beta_{0}$, requires strong-field phenomena, such as those found around neutron stars. Indeed, the two main PPN parameters for such a scalar-tensor theory are

$$
\begin{aligned}
& \gamma^{P P N}-1=-2 \frac{\alpha_{0}^{2}}{1+\alpha_{0}^{2}} \\
& \beta^{P P N}-1=\frac{\beta_{0} \alpha_{0}^{2}}{2\left(1+\alpha_{0}^{2}\right)^{2}} .
\end{aligned}
$$

The deviation of the PPN parameters from the general relativistic values is of second order in $\alpha_{0}$ and of third order in the product $\alpha_{0}^{2} \beta_{0}$. As a result, a very good limit on the parameter $\alpha_{0}$ renders the parameter $\beta_{0}$ practically unconstrainable by weak-field tests.

The study of Damour and Esposito-Farèse [39] revealed one of the main reasons that necessitate careful theoretical studies of possible extensions of general relativity that are suitable for strongfield tests. The order of a term added to the Lagrangian action of the gravitational field is not necessarily a good estimate of the expected magnitude of the observable effects introduced by this additional term. For example, because of the non-linear coupling between the scalar field and matter introduced by the coupling function (23), the deviation from general relativistic predictions is not perturbative. For values of $\beta_{0}$ less than about -6 , it becomes energetically favorable for neutron stars to become "scalarized", with properties that differ significantly from their general relativistic counterparts [39]. Such non-perturbative effects make quantitative tests of strong-field gravity possible even when the astrophysical complications are only marginally understood.

A similar situation, albeit in the opposite regime, arises in an extended gravity theory in which a term proportional to the inverse of the Ricci scalar curvature, $R^{-1}$, is added to the EinsteinHilbert action in order to explain the accelerated expansion of the universe [30]. Although one would expect that such an addition can only affect gravitational fields that are extremely weak, it turns out that it also alters to zeroth order the post-Newtonian parameter $\gamma$ and can, therefore, be excluded by simple solar-system tests [33].

Second, Lagrangian extensions of general relativity often suffer from serious problems with instabilities. This issue can be understood by considering a Lagrangian action that includes terms of second order in the Ricci scalar, i.e., $R^{2}$, as well as the terms of similar order that can be constructed with the Ricci and Riemann tensors. For the sake of the argument, I will consider here the parametric Lagrangian

$$
\mathcal{S}=\frac{c^{4}}{16 \pi G} \int d^{4} x \sqrt{-g}\left(R+\alpha_{2} R^{2}+\beta_{2} R_{\sigma \tau} R^{\sigma \tau}+\gamma_{2} R^{\alpha \beta \gamma \delta} R_{\alpha \beta \gamma \delta}\right),
$$

with $\alpha_{2}, \beta_{2}$, and $\gamma_{2}$ the three parameters of the theory. Such terms arise naturally as highorder corrections in quantum gravity and string theory and their relative importance increases with the curvature of the metric $[50,27]$. They have also been invoked as alternatives to the inflation paradigm for the early expansion of the universe [158]. The predictions for astrophysical objects of extended-gravity theories that incorporate high-order terms have been reported only for a few limited cases in the literature. The dependence of the stellar properties on $R^{2}$ terms in the action has been studied by Parker and Simon [119], who simply derived the generalized Tolmann-Oppenheimer-Volkoff equation without solving it, and by Barraco and Hamity [9], who attempted to solve the problem using a perturbation analysis (unfortunately, this last study suffers from a large number of errors).

This second-order gravity theory has a number of unappealing properties (see discussion in [149, 150]). Classically, a high-order gravity theory requires more than two boundary conditions, which 
is a fact that appears to be incompatible with all other physical theories. Quantum mechanically, second-order gravity theories lead to unstable vacuum solutions. Both these phenomena could be artifacts of the possibility that the action (25) may arise as a low-energy expansion of a nonlocal Lagrangian that is fundamentally of second order [149, 150]. Phenomenologically speaking, these problems can be overcome by requiring the field equations to be of second order, when extremizing the action. This procedure leads to a generalized, high-order gravity theory that remains consistent with classical expectations and is stable quantum-mechanically (according to the procedure outlined in $[149,150])$, but requires a different than usual derivation of the field equations [45].

Even if we neglect these issues, the terms proportional to $\beta_{2}$ and $\gamma_{2}$ lead to field equations with solutions that suffer from the Ostrogradski instability [184]. And even if these terms are dropped and only actions that are generic functions of the Ricci scalar alone are considered, then the resulting solutions for the expansion of the universe [49] and for spherically symmetric stars [144], can be violently unstable, depending on the sign of the second-order term.

A potential resolution to several of these problems in theories with high-order terms in the action appears to be offered by the Palatini formalism. In this approach, the field equations are derived by extremizing the action under variations in the metric and the connection, which is considered as an independent field [155]. For the simple Einstein-Hilbert action, both approaches are equivalent and give rise to the equations of general relativity; when the action has non-linear terms in $R$, the two approaches diverge. Unfortunately, the Palatini formalism leads to equations that cannot handle, in general, the transition across the surface layer of a star to the matter-free space outside it, and is, therefore, not a viable alternative [6].

Finally, it is crucial that we identify the astrophysical phenomena that can be used in testing particular aspects of strong-field gravity. For example, in the case of the classical tests of general relativity, it is easy to show that the deflection of light during a solar eclipse and the Shapiro time delay depend on one (and the same) component of the metric of the Sun (i.e., on the PPN parameter $\gamma$ ). Therefore, they do not provide independent tests of general relativity (as long as we accept the validity of the equivalence principle). On the other hand, the perihelion precession of Mercury and the gravitational redshift depend on the other component of the metric (i.e., on the PPN parameter $\beta$ ) and, therefore, provide complementary tests of the theory. Understanding such degeneracies is an important component of performing tests of gravity theories.

In the case of strong gravitational fields, this issue can be illustrated again by studying the high-order Lagrangian action (25) in the metric formalism (see also [27]). In principle, as the strength of the gravitational field increases, the terms that are of second-order in the Ricci scalar become more important and, therefore, affect the observable properties of neutron stars and black holes. However, because of the Gauss-Bonnet identity,

$$
\frac{\delta}{\delta g_{\mu \nu}} \int d^{4} x\left(R^{2}-4 R_{\sigma \tau} R^{\sigma \tau}+R^{\alpha \beta \gamma \delta} R_{\alpha \beta \gamma \delta}\right)=0
$$

variations, with respect to the metric, of the term proportional to $\gamma_{2}$ in Equation (25), can be expressed as variations of the terms proportional to $\alpha_{2}$ and $\beta_{2}$. Therefore, for all non-quantum gravity tests, the predictions of the theory described by the Lagrangian action (25) are identical to those of the Lagrangian

$$
\mathcal{S}=\frac{c^{4}}{16 \pi G} \int d^{4} x \sqrt{-g}\left[R+\left(\alpha_{2}-\gamma_{2}\right) R^{2}+\left(\beta_{2}+4 \gamma_{2}\right) R_{\sigma \tau} R^{\sigma \tau}\right] .
$$

As a result, astrophysical tests that do not invoke quantum-gravity effects can only constrain a particular combination of the parameters, i.e., $\alpha_{2}-\gamma_{2}$ and $\beta_{2}+4 \gamma_{2}$. It is only through phenomena related to quantum gravity, such as the evaporation of black holes, that the parameter $\gamma$ may be constrained.

Living Reviews in Relativity

http: //www . livingreviews . org/lrr-2008-9 
When the spacetime is isotropic and homogeneous, as in the case of tests using the cosmic evolution of the scale factor, an additional identity is satisfied, i.e.,

$$
\frac{\delta}{\delta g_{\mu \nu}} \int d^{4} x\left(R^{2}-3 R_{\sigma \tau} R^{\sigma \tau}\right)=0 .
$$

This implies that, for cosmological tests, the predictions of the theory described by the Lagrangian action (25) are identical to those of the Lagrangian

$$
\mathcal{S}=\frac{c^{4}}{16 \pi G} \int d^{4} x \sqrt{-g}\left[R+\left(\alpha_{2}+\frac{1}{3} \beta_{2}+\frac{1}{3} \gamma_{2}\right) R^{2}\right] .
$$

As a result, such cosmological tests of gravity can only constrain a particular combination of the parameters, i.e., $\alpha_{2}+\beta_{2} / 3+\gamma_{2} / 3$.

The parameters $\alpha_{2}$ and $\beta_{2}$ can be independently constrained using observations of spacetimes that are strongly curved but not isotropic and homogeneous, such as those found in the vicinities of black holes and neutron stars. Measuring the properties of neutron stars, such as their radii, maximum masses and maximum spins, which require the solution of the field equations in the presence of matter, will provide independent constraints on the combination of parameters $\alpha_{2}-\gamma_{2}$ and $\beta_{2}+4 \gamma_{2}$. However, one can show that in the absence of matter, the external spacetime of a black hole, as given by the solution to Einstein's field equation, is also one (but not necessarily the only) solution of the parametric field equation that arises from the Lagrangian action (25). As a result, tests that involve black holes will probably be inadequate in distinguishing between the particular theory described by Equation (25) and general relativity [130].

This is, in fact, a general problem of using astrophysical observations of black holes to test general relativity in the strong-field regime. The Kerr solution is not unique to general relativity [130]. For example, there is strong analytical [165, 13, 71] and numerical evidence [142] that, in Brans-Dicke scalar-tensor gravity theories, the end product of the collapse of a stellar configuration is a black hole described by the same Kerr solution as in Einstein's theory. The same appears to be true in several other theories generated by adding additional degrees of freedom to Einstein's gravity; the only vacuum solutions that are astrophysically relevant are those described by the Kerr metric [130]. Until a counter-example is discovered, studies of the strong gravitational fields found in the vicinities of black holes can be performed only within phenomenological frameworks, such as those involving multipole expansions of the Schwarzschild and Kerr metrics [138, 35, 66].

To date, it has only been possible to test quantitatively the predictions of general relativity in the strong-field regime using observations of neutron stars, as I will discuss in the following section. In all cases, the general relativistic predictions were contrasted to those of scalar-tensor gravity, with Einstein's theory passing all the tests with flying colors. 


\section{Current Tests of Strong-Field Gravity with Neutron Stars}

Performing tests of strong-field gravity with neutron stars requires knowledge of the equation of state of neutron-star matter to a degree better than the required precision of the gravitational test. This appears from the outset to be a serious hurdle given the wide range of predictions of equally plausible theories of neutron-star matter (see [83] for a recent compilation). It is easy to show, however, that current uncertainties in our modeling of the properties of ultra-dense matter do not preclude significant constraints on the strong-field behavior of gravity [43].

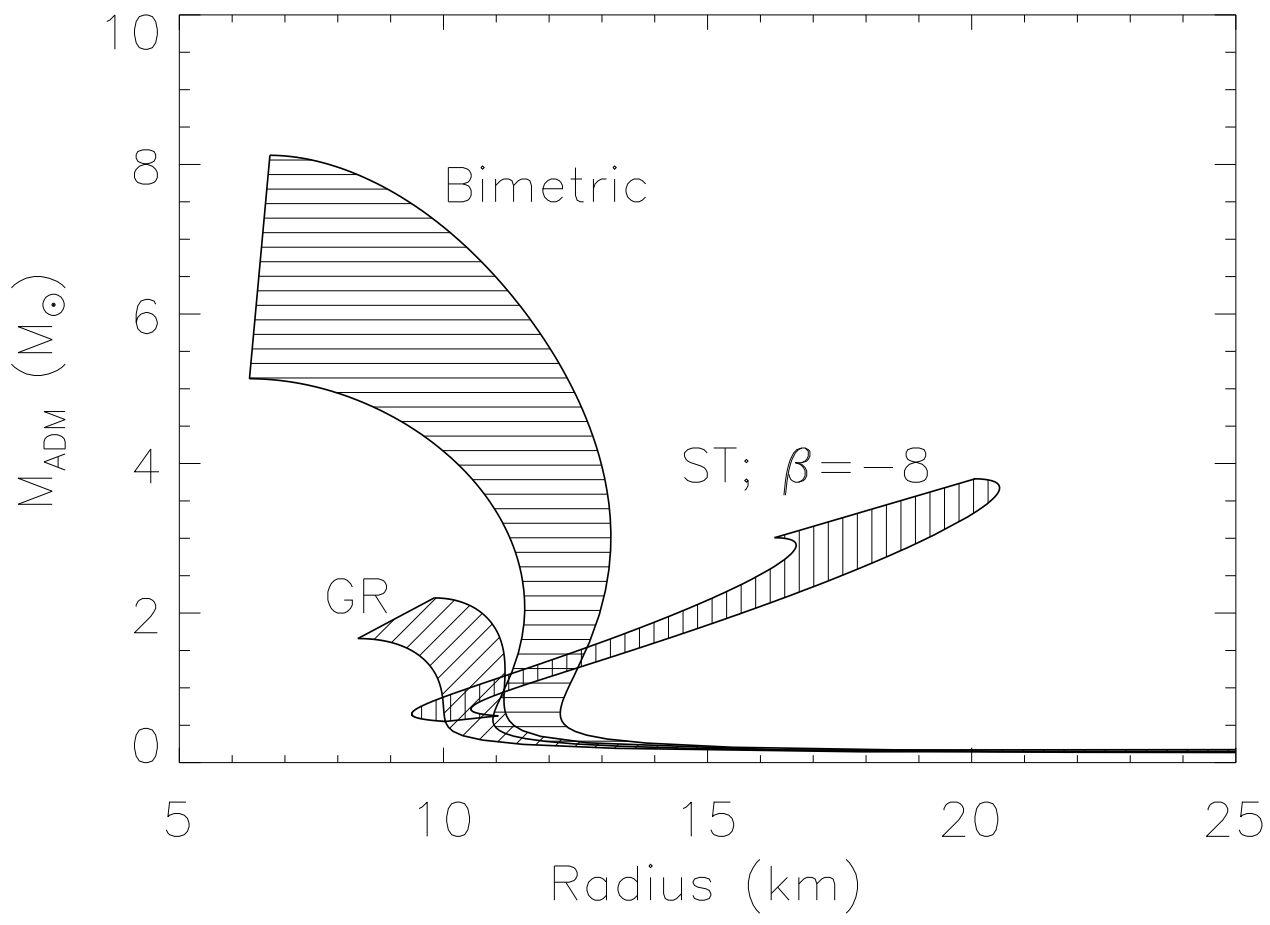

Figure 13: Mass-radius relations of neutron stars in general relativity (GR), scalar-tensor gravity (ST), and Rosen's bimetric theory of gravity [43]. The shaded areas represent the range of mass-radius relations predicted in each case by neutron-star equations of state without unconfined quarks or condensates. All gravity theories shown in the figure are consistent with solar-system tests but introduce variations in the predicted sizes of neutron stars that are significantly larger than the uncertainty caused by the unknown equation of state.

During the last three decades, neutron-star models have been calculated for a variety of gravity theories (see [180] and references therein) and were invariably different, both in size and in allowed mass, than their general relativistic counterparts. As an example, Figure 13 shows neutron-star models calculated in three representative theories that cannot be excluded by current tests that do not involve neutron stars. In the figure, the shaded areas represent the uncertainty introduced by the unknown equation of state of neutron-star matter (not including quark stars or large neutron stars with condensates). Clearly, the deviations in neutron-star properties from the predictions of general relativity for these theories (that are still consistent with weak-field tests) are larger than the uncertainty introduced by the unknown equation of state of neutron-star matter.

This is a direct consequence of the fact that the curvature around a neutron star is larger by

Living Reviews in Relativity

http: //www . livingreviews . org/lrr-2008-9 
$\sim 13$ orders of magnitude compared to the curvature probed by solar-system tests, whereas the density inside the neutron star is larger by only an order of magnitude compared to the densities probed by nuclear scattering data that are used to constrain the equation of state. Given that the current values of the post-Newtonian parameters are known from weak-field tests to within $\sim 10^{-5}$, it is reasonable that deviations from general relativity can be hidden in the weak-field limit but may become dominant as the curvature is increased by more than ten orders of magnitude. Neutron stars can indeed be used in testing the strong-field behavior of a gravity theory.

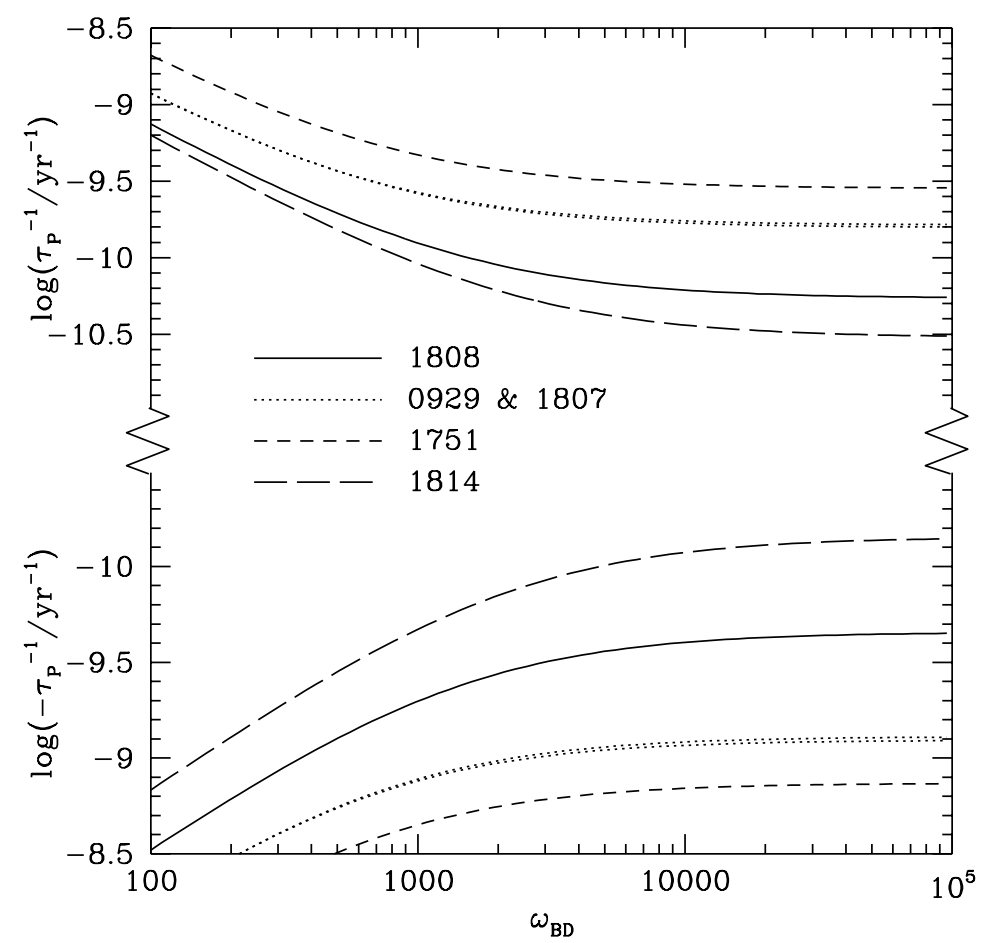

Figure 14: The limiting rate for the evolution of the orbital periods $\left(\tau_{\mathrm{P}}^{-1} \equiv \dot{P} / P\right)$ of five known millisecond, accreting pulsars as a function of the Brans-Dicke parameter $\omega_{\mathrm{BD}}$. The lower half of the plot corresponds to an orbital period that decreases with time $(\dot{P} / P<0)$, whereas the upper half corresponds to an orbital period that increases with time $(\dot{P} / P>0)$. Only the area outside the two curves for each system is physically allowed [125].

\subsection{Brans-Dicke gravity and the orbital decay of binary systems with neutron stars}

Binary stellar systems that are currently known to harbor at least one neutron star have orbital separations that are too large to be used in probing directly strong gravitational fields. Even at that separation, however, the orbital evolution of the binary system caused by the emission of gravitational waves is affected, in a scalar-tensor theory, by the coupling of matter to the scalar field, which occurs in a strong gravitational field. This manifests itself as a violation of the strong equivalence principle, with many observable consequences such as the rapid decay of the orbit due to emission of dipole radiation $[54,182]$. The various quantitative tests of strong-field gravity using binary systems with radio pulsars have been reviewed in detail elsewhere [157]. Here, I will focus 
only on tests that involve the orbital period evolution of the binary systems.

The best studied binaries with compact objects are the double neutron stars, with the HulseTaylor pulsar (PSR 1913+16) as the prototypical case. Unfortunately, in all double neutron-star systems, the masses of the two members of the binary are surprisingly similar [166] and this severely limits the prospects of placing strong constraints on the dipole radiation from them. Indeed, the magnitude of dipole radiation depends on the difference of the sensitivities between the two members of the binaries, and for neutron stars the sensitivities depend primarily on their masses. The resulting constraint imposed on the Brans-Dicke parameter $\omega$ by the Hulse-Taylor pulsar is significantly smaller than the limit $\omega>40,000$ set by the Cassini mission [15].

The constraint is significantly improved when studying binary systems in which only one of the two stars is a neutron star. There are several known neutron star-white dwarf binaries that are suitable for this purpose, in which the neutron stars appear as radio pulsars (e.g., PSR B0655+64 [40], PSR J0437-4715 [172]), as millisecond accreting X-ray pulsars (e.g., XTE J1808-456 [125]), or as non-pulsing X-ray sources (e.g., 4U 1820-30 [182]). In the last two cases, the evolution of the binary orbit is also affected significantly by mass transfer from the companion star to the neutron star. However, for each value of the Brans-Dicke parameter $\omega_{\mathrm{BD}}$, there is a minimum absolute value for the rate of evolution of the orbital period (see Figure 14 and [125]). An accurate measurement of the orbital period derivative in any of these systems offers, therefore, the potential of placing a lower limit on the Brans-Dicke parameter. Because of the astrophysical complications introduced by mass transfer, the optimal constraint on $\omega_{\mathrm{BD}}$ is of order $10^{4}$ in this method, which is comparable to the Cassini limit.

\subsection{Second-order scalar-tensor gravity and radio pulsars}

As discussed in the previous section, observations of strong-field phenomena provide constraints on Brans-Dicke scalar-tensor gravity, which are, however, at most comparable to those of solar system tests. This is true because the fractional deviation of a Brans-Dicke theory from general relativity is of order $\omega_{\mathrm{BD}}^{-1}$, both for weak and strong gravitational fields, and the solar-system tests have superb accuracy. On the other hand, a scalar-tensor theory with a second-order coupling (e.g., the one arising from the action (22) with the coupling (23)) allows for large deviations in the strong-field regime, while being consistent with the weak-field limits [39, 40].

In the case of neutron stars, the second-order scalar-tensor theory described by Damour and Esposito-Farèse [39] leads to a non-perturbative effect known as spontaneous scalarization (similar to the spontaneous magnetization in ferromagnetism). For significantly large negative values of the parameter $\beta_{0}$, there is a range of neutron-star masses for which it becomes energetically favorable for the scalar field to acquire high values inside the neutron star and affect its structure significantly compared to the general relativistic predictions. An example of the mass-radius relation for neutron stars in a second-order scalar-tensor theory with $\beta_{0}=-8$ is shown in Figure 13.

The properties and stability of scalarized neutron stars have been studied extensively in the literature [39, 139, 70]. For the purposes of tests of strong-field gravity, the coupling of matter with the gravitational field and the external spacetimes of scalar stars are so different compared to their general relativistic counterparts that large negative values of $\beta_{0}$ can be firmly excluded with current observations of binary stellar systems that harbor radio pulsars (see Figure 15).

As expected, weak-field tests bound significantly the value of the parameter $\alpha_{0}$, leaving the parameter $\beta_{0}$ largely unconstrained. Between the binary systems with radio pulsars, the one with the white-dwarf companion provides the most stringent constraints because the large asymmetry between the two compact objects leads to the prediction of strong dipole gravitational radiation that can be excluded observationally. Finally, for large negative values of the parameter $\beta_{0}$, the scalarization of the neutron stars makes the predictions of the theory incompatible with observations.

Living Reviews in Relativity

http: //www. livingreviews.org/lrr-2008-9 


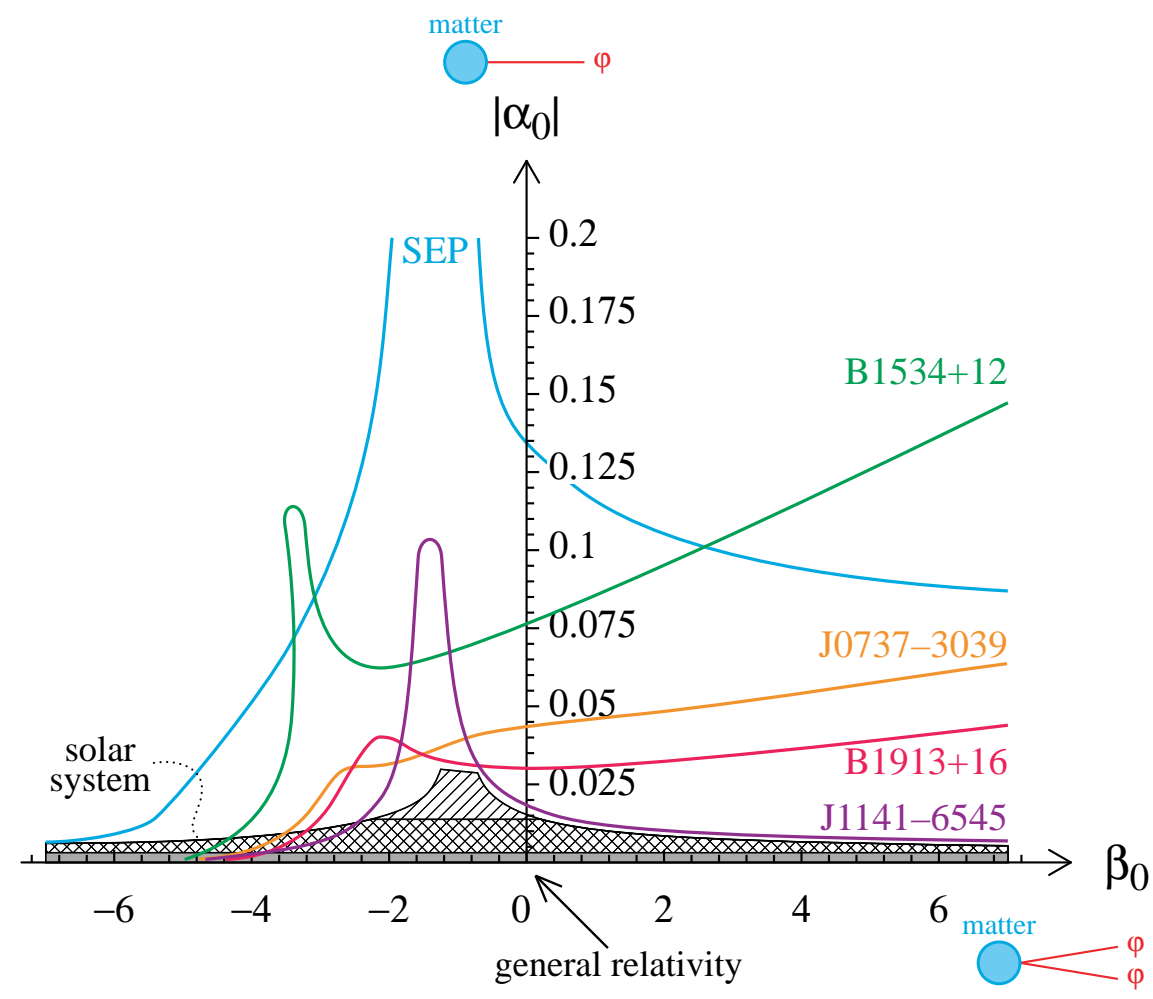

Figure 15: Constraints on the two parameters of a second-order scalar-tensor theory placed by the timing properties of binary stellar systems that harbor neutron stars (SEP stands for tests of the strong equivalence principle). The constraints imposed by solar system tests, including the Cassini mission, are also shown for comparison [38]. In all cases, the allowed part of the parameter space is under the corresponding curve. 


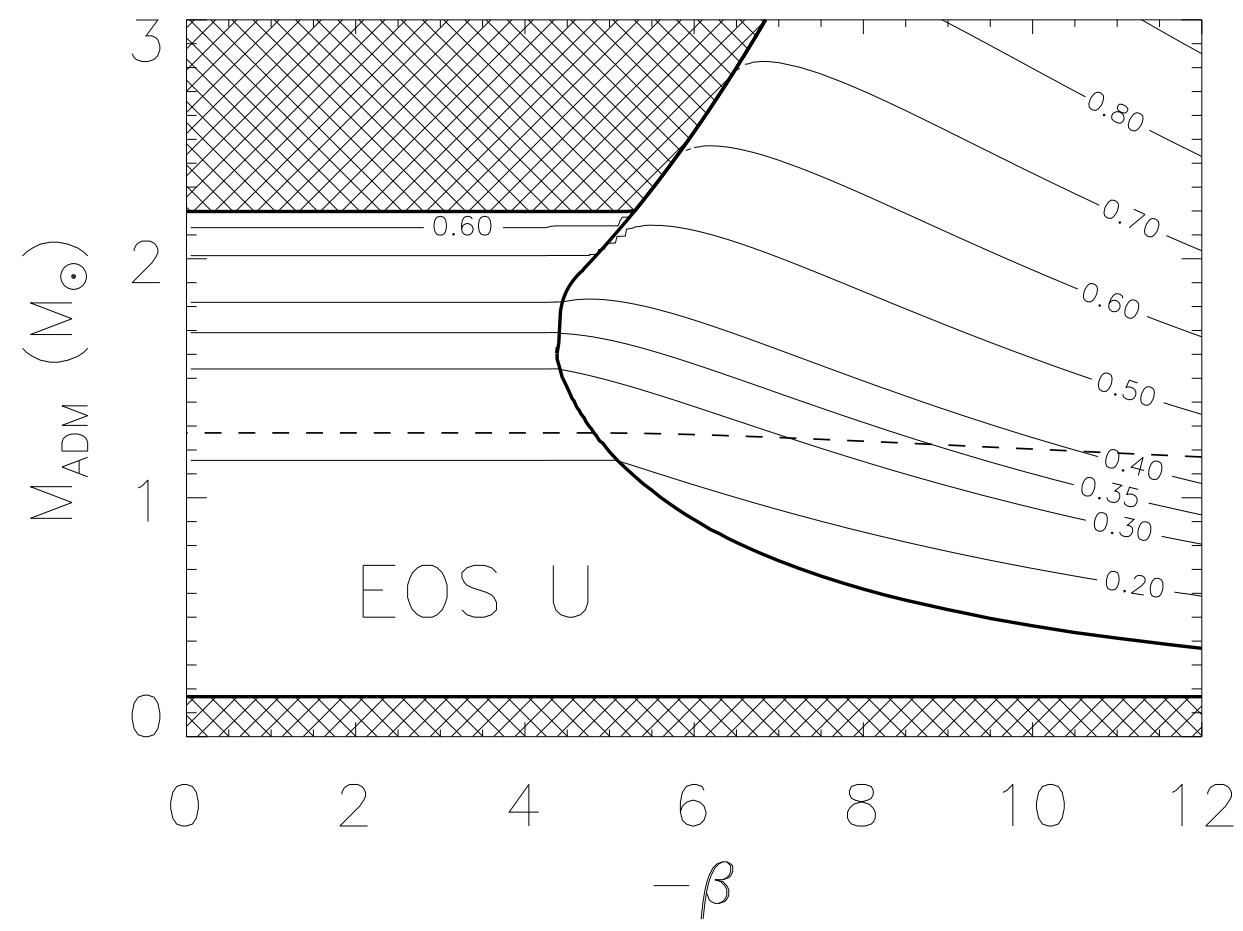

Figure 16: Contours of constant gravitational redshift measured at infinity for an atomic line originating at the surface of a neutron star in a scalar-tensor gravity theory, for different values of the parameter $\beta_{0}$ that measures the relative contribution of the scalar field. The thick curve separates the scalarized stars from their general-relativistic counterparts. The measurements of a redshift of $z=0.35$ from a burster [37] and the astrophysical constraint of a baryonic mass of at least $1.4 M_{\odot}$ (dashed lines) result in a bound on the parameter $\beta$ of $-\beta<9$ [43]. 


\subsection{Second-order scalar-tensor gravity and X-ray observations of accret- ing neutron stars}

The quantitative features of a number of phenomena observed in the X-rays from accreting neutron stars depend strongly on their masses and radii, as discussed in 3 . The constraints imposed by two of these phenomena on the parameters of the second-order scalar-tensor gravity of Damour and Esposito-Farèse [39] have been studied recently [43, 44].

The first phenomenon is the observation of gravitationally-redshifted atomic lines during X-ray bursts from the source EXO 0748-676 [37]. Figure 16 shows the values of the gravitational redshift from the surface of neutron stars with different masses in second-order scalar-tensor theories with different values of the parameter $\beta_{0}$ [43]. In this calculation, the parameter $\alpha_{0}$ was set to zero and the neutron-star structure was calculated using the equation of state $\mathrm{U}$ [36]. The hatch-filled area corresponds to neutron-star masses that are unacceptable for each value of the parameter $\beta_{0}$, while the thick curve separates the scalarized stars from their general-relativistic counterparts.

A dynamical measurement of the mass of EXO 0748-676 can rule out the possibility that the neutron star in this source is scalarized, because scalarized stars have very different surface redshifts compared to the general-relativistic stars of the same mass. The source EXO 0748-676 lies in an eclipsing binary system, which makes it a prime candidate for a dynamical mass measurement. In the absence of such a measurement, however, a limit on the parameter $\beta_{0}$ can be placed under the astrophysical constraint that the baryonic mass of the neutron stars is larger than $\simeq 1.4 M_{\odot}$. This is a reasonable assumption, given that a progenitor core of a lower mass would not have collapsed to form a neutron star. Combining this constraint with the measured redshift of $z=0.35$ leads to a limit on the parameter $-\beta_{0}<9$, which depends only weakly on the assumed equation of state of neutron-star matter [43].

A second set of phenomena that can lead to strong-field tests of gravity are the fast quasiperiodic oscillations observed from many bright accreting neutron stars [171]. The highest known frequency of such an oscillations is $1330 \mathrm{~Hz}$, observed from the source 4U 1636-53 and corresponding to the Keplerian frequency of the innermost stable circular orbit of a $1.6 M_{\odot}$ slowly-spinning neutron star. Figure 17 shows the maximum Keplerian frequency outside a neutron star in the second-order scalar tensor theory for different values of the parameter $\beta_{0}$. For small stellar masses, the limiting frequency is achieved at the surface of the star, whereas for large stellar masses, the limiting frequency is reached at the innermost stable circular orbit. Figure 17 shows that scalarized stars allow for higher frequencies than their general-relativistic counterparts. Therefore, requiring the observed oscillation frequency to be smaller than the highest Keplerian frequency of a stable orbit outside the compact object cannot be used to constrain the parameters of this theory. On the other hand, the correlations between the various dynamical frequencies outside the compact object depend strongly on the parameter $\beta$ and hence the gravity theory can be constrained given a particular model for the oscillations [44]. 


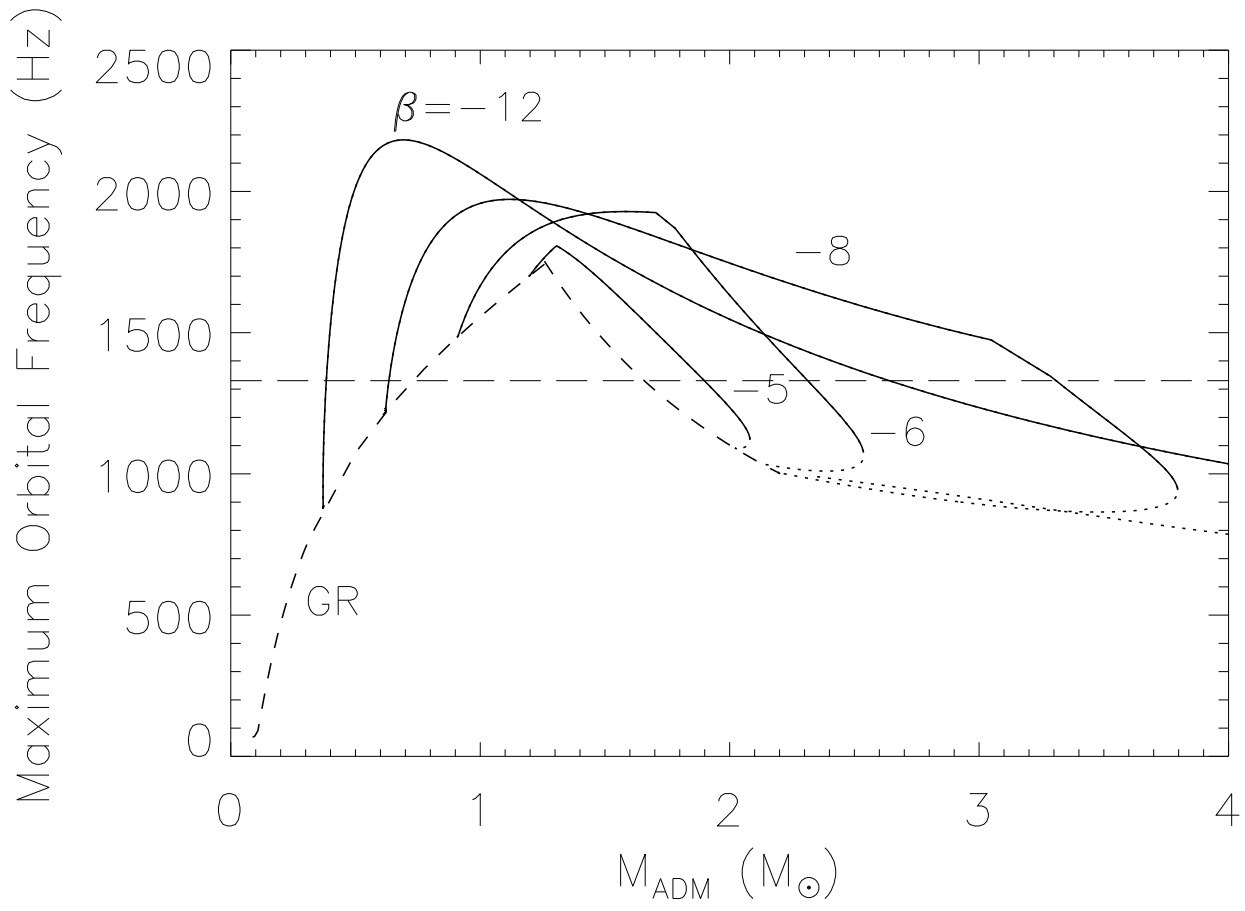

Figure 17: The maximum orbital frequency outside a neutron star of mass $M_{\text {ADM }}$ for different scalartensor theories identified by the parameter $\beta$. The dashed line shows the maximum observed frequency of a quasi-periodic oscillation from an accreting neutron star [44]. 


\section{Going Beyond Einstein}

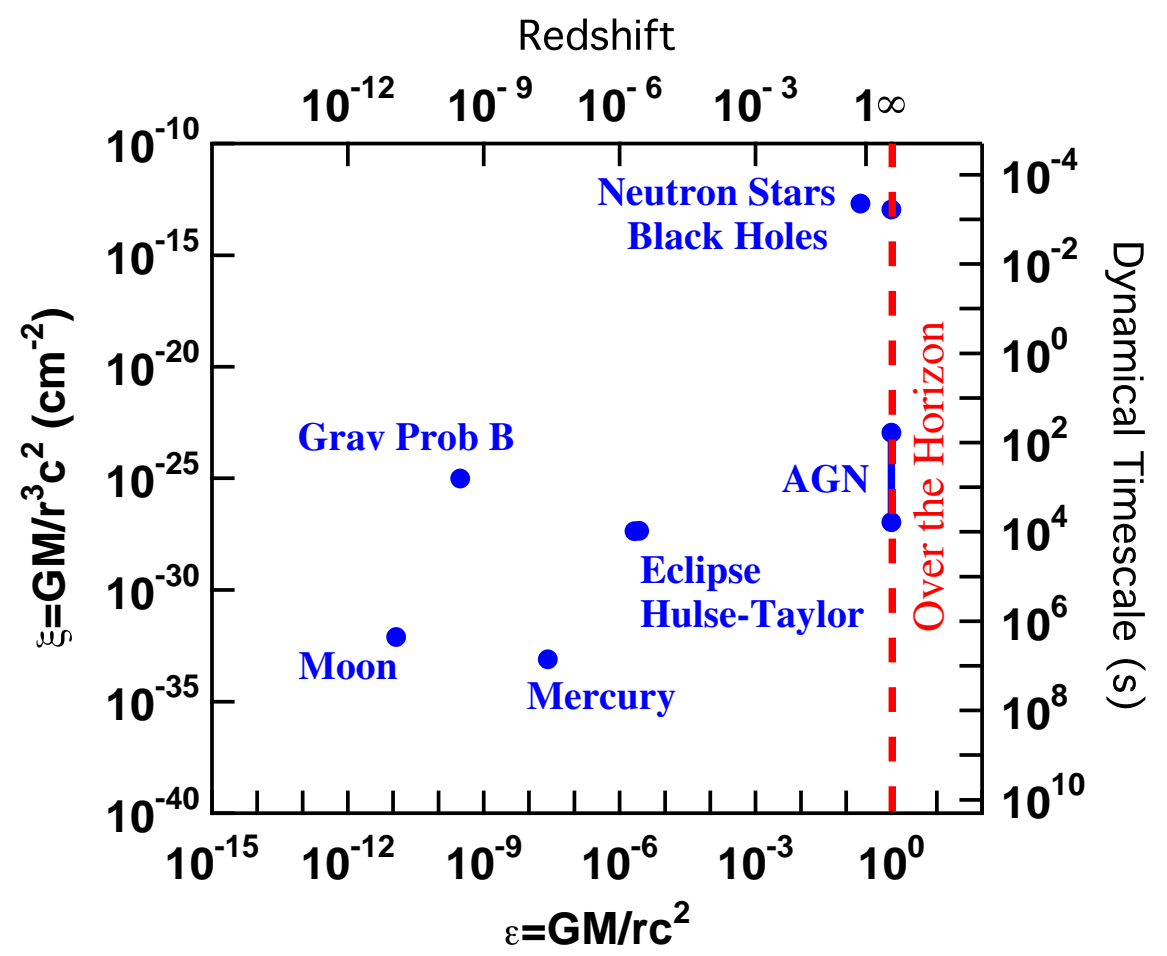

Figure 18: The spectral (redshift) and timing capabilities required for an observatory to probe different strengths of gravitational fields. Phenomena that occur in the vicinities of neutron stars and stellar-mass black holes experience large redshift and occur over sub-millisecond timescales.

Testing general relativity in the strong-field regime with neutron stars and black holes will require advanced observatories that will be able to resolve various phenomena in the characteristic energy and time scales in which they occur. The two parameters used to quantify the strength of a gravitational field in Section 3.1 are also useful in discussing the specifications required for such future observatories.

The potential and the curvature in a gravitational field are directly related, respectively, to the characteristic energy and time scales that need to be resolved in order for an observation to be able to probe a particular region of the parameter space. The potential $\epsilon$ directly gives the gravitational redshift $z$ according to

$$
z=1-(1-2 \epsilon)^{-1 / 2}
$$

the measurement of which is the goal of spectroscopic observations; for weak gravitational fields $z \simeq \epsilon$. At the same time, the curvature $\xi$ is directly related to the dynamical timescale $\tau$ in the same region of a gravitational field by

$$
\tau=\frac{2 \pi}{c} \xi^{-1 / 2}
$$

As shown in Figure 18, only observatories with excellent spectroscopic and millisecond timing capabilities will be able to resolve phenomena that occur in the strongest gravitational fields found in astrophysics, i.e., those in the vicinities of neutron stars and stellar-mass black holes. 


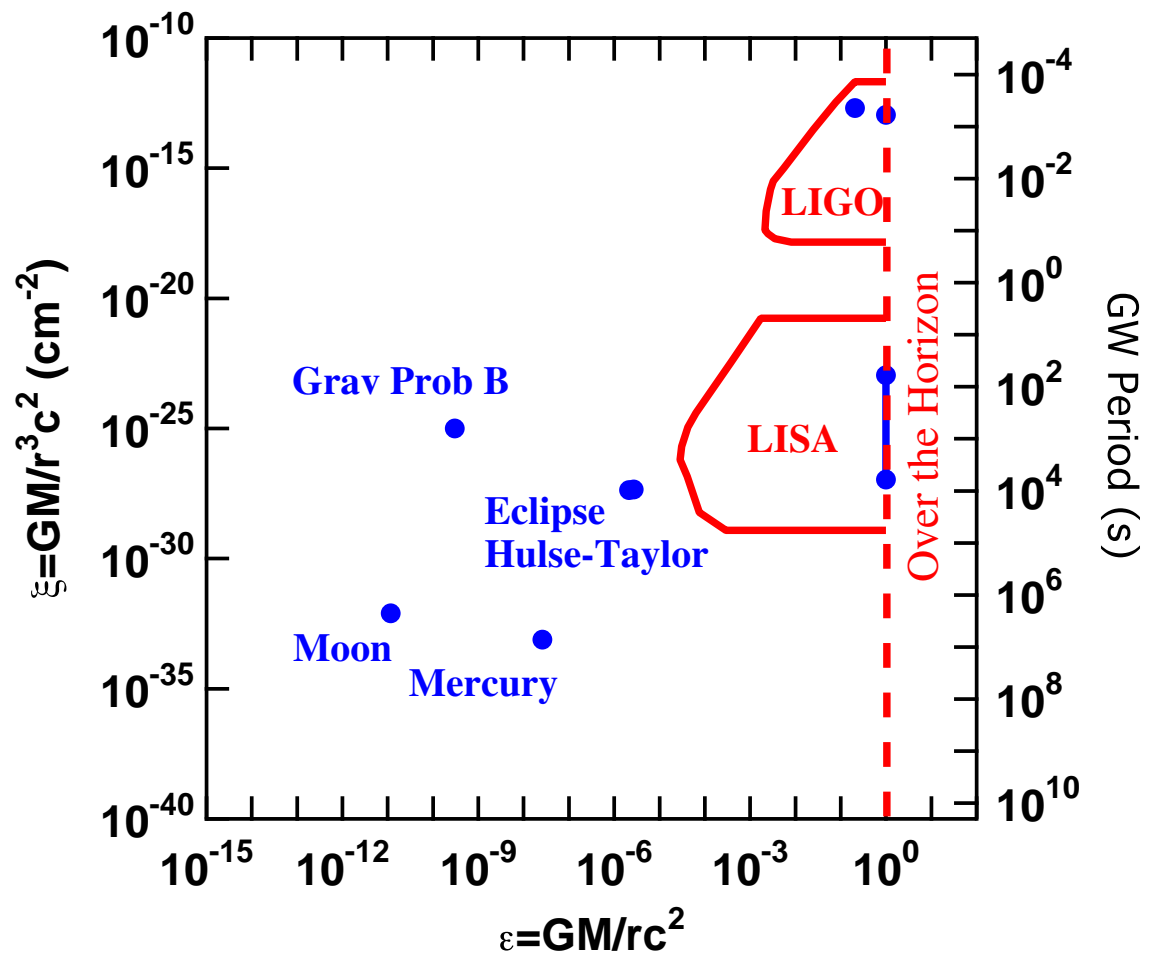

Figure 19: The parameter space that will be probed by an experiment based on gravitational wave detection with LIGO and LISA, for an assumed source at a distance of $1 \mathrm{Mpc}$. 
One of the most promising avenues of testing strong-field general relativity is via the detection of the gravitational waves emitted during the coalescence of compact objects. In the simple case in which two compact objects of mass $M$ are orbiting each other in circular orbits with separation $a$, slowly approaching because of the emission of gravitational waves, the characteristic period $P$ of the gravitational wave is half of the orbital period and, therefore, is related to the spacetime curvature by

$$
P=\frac{\pi}{c} \xi^{-1 / 2} .
$$

At the same time, the strain $h$ detected by an observatory on Earth for a gravitational wave emitted by such a source placed at a distance $D$, is [58]

$$
h=\left(\frac{G M}{a c^{2}}\right)\left(\frac{G M}{D c^{2}}\right) .
$$

Given the distance to the source and the measurement of strain, the curvature of the gravitational field probed is

$$
\xi=\frac{\epsilon^{5}}{h^{2} D^{2}}=10^{-3} \epsilon^{5}\left(\frac{h}{10^{-23}}\right)^{-2}\left(\frac{D}{1 \mathrm{Mpc}}\right)^{-2} \mathrm{~cm}^{-2} .
$$

The sensitivity of each gravitational wave detector depends strongly on the period of the wave. Using Equations (32) and (34), the sensitivity curve of a detector can be converted into a region of the parameter space that can be probed, given the distance to the source. This is shown in Figure 19 for the advanced LIGO and LISA for an assumed source distance of $1 \mathrm{Mpc}$. Gravitational waves detected by LISA will probe the same curvatures as current tests of general relativity, but significantly larger potentials. On the other hand, gravitational waves detected by the advanced LIGO have the potential to directly probe directly the strongest gravitational fields found around astrophysical objects.

In the near future, a number of observatories will exploit new techniques and open new horizons in gravitational physics by exploring the strong-field region of the parameter space shown in Figure 18. Observations with the Square Kilometre Array [151] may lead to the discovery of the most optimal binary systems for strong-field gravity tests with pulsar timing, in which a pulsar is orbiting a black hole [78]. High-energy observations of black holes and neutron stars with IXO [74] and XEUS [185] will detect highly-redshifted atomic lines and measure their rapid variability properties. Finally, gravitational wave observatories, either from the ground (such as LIGO [88], GEO600 [62], TAMA300 [163] and VIRGO [175]) or from space (such as LISA [108]) will directly detect, for the first time, one of the most remarkable predictions of general relativity, the generation of gravitational waves from orbiting compact objects and black-hole ringing. 


\section{Acknowledgements}

It is my great pleasure to acknowledge many fruitful discussions and collaborations with a number of people that have shaped my ideas on astrophysical tests of strong-field gravity. In particular, I thank T. Belloni, D. Chakrabarty, S. DeDeo, F. Lamb, C. Miller, R. Narayan, J. McClintock, F. Özel and M. van der Klis. I am indebted to S. DeDeo and F. Özel for helping me settle on and understand the definition of strong-field gravity. I am also grateful to G. Esposito-Farèse, T. Johanssen, J. McClintock, F. Özel and C. Reynolds for their detailed comments that helped me greatly improve the presentation of this review. 


\section{References}

[1] Abramowicz, M.A., and Kluźniak, W., "A precise determination of black hole spin in GRO J1655-40", Astron. Astrophys., 374, L19-L20, (2001). Related online version (cited on 24 July 2007): http://arXiv.org/abs/astro-ph/0105077. 4.4.2, 12

[2] Abramowicz, M.A., Kluźniak, W., McClintock, J.E., and Remillard, R.A., "The Importance of Discovering a 3:2 Twin-Peak Quasi-periodic Oscillation in an Ultraluminous X-Ray Source, or How to Solve the Puzzle of Intermediate-Mass Black Holes", Astrophys. J. Lett., 609, L63L65, (2004). Related online version (cited on 24 July 2007): http://arXiv.org/abs/astro-ph/0402012. 4.4.2

[3] Agol, E., and Krolik, J.H., "Magnetic Stress at the Marginally Stable Orbit: Altered Disk Structure, Radiation, and Black Hole Spin Evolution", Astrophys. J., 528, 161-170, (2000). ADS: http://adsabs.harvard.edu/abs/2000ApJ ...528. .161A. 4.2.3, 4.2.3

[4] Armitage, P.J., and Reynolds, C.S., "The variability of accretion on to Schwarzschild black holes from turbulent magnetized discs", Mon. Not. R. Astron. Soc., 341, 1041-1050, (2003). ADS: http://adsabs.harvard.edu/abs/2003MNRAS.341.1041A. 4.3.2

[5] Balbus, S.A., and Papaloizou, J.C.B., "On the Dynamical Foundations of alpha Disks", Astrophys. J., 521, 650-658, (1999).

ADS: http://adsabs.harvard.edu/abs/1999ApJ...521_.650B. 4.2.3

[6] Barausse, E., Sotiriou, T.P., and Miller, J.C., "Curvature singularities, tidal forces and the viability of Palatini $f(R)$ gravity", Class. Quantum Grav., 25, 105008, (2008). Related online version (cited on 29 May 2008):

http://arXiv.org/abs/0712.1141. 5

[7] Bardeen, J.M., "Timelike and null geodesics in the Kerr metric", in DeWitt, C., and DeWitt, B.S., eds., Black Holes, Based on lectures given at the 23rd session of the Summer School of Les Houches, 1972, pp. 215-239, (Gordon and Breach, New York, U.S.A., 1973). 4.1

[8] Bardeen, J.M., Press, W.H., and Teukolsky, S.A., "Rotating Black Holes: Locally Nonrotating Frames, Energy Extraction, and Scalar Synchrotron Radiation", Astrophys. J., 178, 347-369, (1972).

ADS: http://adsabs .harvard.edu/abs/1972ApJ ...178. .347B. 4.4.1

[9] Barraco, D.E., and Hamity, V.H., "Stellar model in a fourth order theory of gravity", Phys. Rev. D, 57, 954-960, (1998). 5

[10] Barret, D., Olive, J.-F., and Miller, M.C., "The coherence of kilohertz quasi-periodic oscillations in the X-rays from accreting neutron stars", Mon. Not. R. Astron. Soc., 370, 1140-1146, (2006). Related online version (cited on 24 July 2007):

http://arXiv.org/abs/astro-ph/0605486. 11, 4.4.1

[11] Barret, D., Olive, J.-F., and Miller, M.C., "Supporting evidence for the signature of the innermost stable circular orbit in Rossi X-ray data from 4U 1636-536", Mon. Not. R. Astron. Soc., 376, 1139-1144, (2007). 4.4.1

[12] Beckwith, K., and Done, C., "Iron line profiles in strong gravity", Mon. Not. R. Astron. Soc., 352, 353-362, (2004). Related online version (cited on 24 July 2007):

http://arXiv.org/abs/astro-ph/0402199. 4.3.2 
[13] Bekenstein, J.D., "Nonexistence of Baryon Number for Black Holes. II", Phys. Rev. D, 5, 2403-2412, (1972). 3.3, 5

[14] Bekenstein, J.D., "The modified Newtonian dynamics-MOND and its implications for new physics", Contemp. Phys., 47, 387-403, (2007). Related online version (cited on 24 July 2007):

http://arXiv.org/abs/astro-ph/0701848. 3.2

[15] Bertotti, B., Iess, L., and Tortora, P., "A test of general relativity using radio links with the Cassini spacecraft", Nature, 425, 374-376, (2003). 6.1

[16] NASA, "Beyond Einstein", project homepage. URL (cited on 05 July 2007): http://universe.nasa.gov/. 1

[17] Bhattacharyya, S., Miller, M.C., and Lamb, F.K., "The Shapes of Atomic Lines from the Surfaces of Weakly Magnetic Rotating Neutron Stars and Their Implications", Astrophys. J., 644, 1085-1089, (2006). Related online version (cited on 24 July 2007): http://arXiv.org/abs/astro-ph/0412107. 4.3.1

[18] Bildsten, L., and Rutledge, R.E., "Coronal X-Ray Emission from the Stellar Companions to Transiently Accreting Black Holes", Astrophys. J., 541, 908-917, (2000).

ADS: http://adsabs.harvard.edu/abs/2000ApJ...541 . 908B. 4.2.1

[19] Bildsten, L., Salpeter, E.E., and Wasserman, I., "The fate of accreted CNO elements in neutron star atmospheres: X-ray bursts and gamma-ray lines", Astrophys. J., 384, 143-176, (1992).

ADS: http://adsabs.harvard.edu/abs/1992ApJ . . 384 . .143B. 4.3.1

[20] Blaes, O.M., Davis, S.W., Hirose, S., Krolik, J.H., and Stone, J.M., "Magnetic Pressure Support and Accretion Disk Spectra", Astrophys. J., 645, 1402-1407, (2006). Related online version (cited on 24 July 2007):

http://arXiv.org/abs/astro-ph/0601380. 4.2.3

[21] Braje, T.M., and Romani, R.W., "RX J1856-3754: Evidence for a Stiff Equation of State", Astrophys. J., 580, 1043-1047, (2002).

ADS: http://adsabs.harvard.edu/abs/2002ApJ...580.1043B. 4.3.1

[22] Brans, C., and Dicke, R.H., "Mach's Principle and a Relativistic Theory of Gravitation", Phys. Rev., 124, 925-935, (1961). 2

[23] Brenneman, L.W., and Reynolds, C.S., "Constraining Black Hole Spin via X-Ray Spectroscopy", Astrophys. J., 652, 1028-1043, (2006). Related online version (cited on 24 July 2007):

http://arXiv.org/abs/astro-ph/0608502. 8, 4.3.2

[24] Broderick, A.E., and Loeb, A., "Frequency-dependent Shift in the Image Centroid of the Black Hole at the Galactic Center as a Test of General Relativity", Astrophys. J., 636, L109-L112, (2006). Related online version (cited on 24 July 2007): http://arXiv.org/abs/astro-ph/0508386. 4.1

[25] Broderick, A.E., and Loeb, A., "Testing General Relativity with High-Resolution Imaging of Sgr A*", J. Phys.: Conf. Ser., 54, 448-455, (2006). URL (cited on 29 May 2008): http://stacks.iop.org/1742-6596/54/448. 4.1 
Probes and Tests of Strong-Field Gravity with Observations in the Electromagnetic Spectrum 49

[26] Brown, E.F., Bildsten, L., and Rutledge, R.E., "Crustal Heating and Quiescent Emission from Transiently Accreting Neutron Stars", Astrophys. J., 504, L95-L98, (1998).

ADS: http://adsabs .harvard.edu/abs/1998ApJ ... 504L. .95B. 4.2.1

[27] Burgess, C.P., "Quantum Gravity in Everyday Life: General Relativity as an Effective Field Theory", Living Rev. Relativity, 7, lrr-2004-5, (2004). URL (cited on 05 July 2007): http://www.livingreviews.org/lrr-2004-5. 1, 5

[28] Cadeau, C., Morsink, S.M., Leahy, D., and Campbell, S.S., "Light Curves for Rapidly Rotating Neutron Stars", Astrophys. J., 654, 458-469, (2007). Related online version (cited on 24 July 2007):

http://arXiv.org/abs/astro-ph/0609325. 4.3.1

[29] Carroll, S.M., "The Cosmological Constant", Living Rev. Relativity, 4, lrr-2001-1, (2001). URL (cited on 05 July 2007):

http://www.livingreviews.org/lrr-2001-1. 1

[30] Carroll, S.M., Duvvuri, V., Trodden, M., and Turner, M.S., "Is cosmic speed-up due to new gravitational physics?", Phys. Rev. D, 70, (2004). Related online version (cited on 24 July 2007):

http://arXiv.org/abs/astro-ph/0306438. 5

[31] Carroll, S.M., and Kaplinghat, M., "Testing the Friedmann equation: The expansion of the universe during big-bang nucleosynthesis", Phys. Rev. D, 65, 063507, (2002). Related online version (cited on 24 July 2007):

http://arXiv.org/abs/astro-ph/0108002. 3.2

[32] Chang, P., Morsink, S.M., Bildsten, L., and Wasserman, I., "Rotational Broadening of Atomic Spectral Features from Neutron Stars", Astrophys. J., 636, L117-L120, (2006). Related online version (cited on 24 July 2007):

http://arXiv.org/abs/astro-ph/0511246. 4.3.1

[33] Chiba, T., "1/R gravity and scalar-tensor gravity", Phys. Lett. B, 575, 1-3, (2003). Related online version (cited on 24 July 2007):

http://arXiv.org/abs/astro-ph/0307338. 5

[34] Clayton, D.D., Principles of stellar evolution and nucleosynthesis, (University of Chicago Press, Chicago, U.S.A., 1983). 3.2

[35] Collins, N.A., and Hughes, S.A., "Towards a formalism for mapping the spacetimes of massive compact objects: Bumpy black holes and their orbits", Phys. Rev. D, 69, 124022, (2004). Related online version (cited on 24 July 2007):

http://arXiv.org/abs/gr-qc/0402063. 5

[36] Cook, G.B., Shapiro, S.L., and Teukolsky, S.A., "Rapidly rotating neutron stars in general relativity: Realistic equations of state", Astrophys. J., 424, 823-845, (1994).

ADS: http://adsabs.harvard.edu/abs/1994ApJ . . .424 . .823C. 6.3

[37] Cottam, J., Paerels, F., and Mendez, M., "Gravitationally redshifted absorption lines in the X-ray burst spectra of a neutron star", Nature, 420, 51-54, (2002). Related online version (cited on 24 July 2007): http://arXiv.org/abs/astro-ph/0211126. 4.3.1, 16, 6.3 
[38] Damour, T., "Binary Systems as Test-beds of Gravity Theories", (2007). URL (cited on 24 July 2007):

http://arxiv.org/abs/0704.0749. 15

[39] Damour, T., and Esposito-Farese, G., "Nonperturbative strong-field effects in tensor-scalar theories of gravitation", Phys. Rev. Lett., 70, 2220-2223, (1993). 1, 5, 5, 5, 6.2, 6.3

[40] Damour, T., and Esposito-Farèse, G., "Tensor-scalar gravity and binary-pulsar experiments", Phys. Rev. D, 54, 1474-1491, (1996). Related online version (cited on 24 July 2007): http://arXiv.org/abs/gr-qc/9602056. 3.3, 6.1, 6.2

[41] Davis, S.W., Blaes, O.M., Hubeny, I., and Turner, N.J., "Relativistic Accretion Disk Models of High-State Black Hole X-Ray Binary Spectra", Astrophys. J., 621, 372-387, (2005). Related online version (cited on 24 July 2007):

http://arXiv.org/abs/astro-ph/0408590. 7, 4.2.3, 4.2.3

[42] De Villiers, J.-P., and Hawley, J.F., "A Numerical Method for General Relativistic Magnetohydrodynamics", Astrophys. J., 589, 458-480, (2003).

ADS: http://adsabs.harvard.edu/abs/2003ApJ . .589. .458D. 4.2.3

[43] Dedeo, S., and Psaltis, D., "Towards New Tests of Strong-Field Gravity with Measurements of Surface Atomic Line Redshifts from Neutron Stars", Phys. Rev. Lett., 90, 141101, (2003). Related online version (cited on 24 July 2007):

http://arXiv.org/abs/astro-ph/0302095. 5, 6, 13, 16, 6.3

[44] DeDeo, S., and Psaltis, D, "Testing Strong-field Gravity with Quasi-Periodic Oscillations", Phys. Rev. D, submitted, (2007). Related online version (cited on 05 July 2007): http://arXiv .org/abs/astro-ph/0405067. 5, 6.3, 6.3, 17

[45] DeDeo, S., and Psaltis, D., "Stable, accelerating universes in modified-gravity theories", Phys. Rev. D, 78, 064013, (2008).

ADS: http://adsabs.harvard.edu/abs/2008PhRvD. .78f4013D. 5

[46] Dehnen, W., and Binney, J., "Mass models of the Milky Way", Mon. Not. R. Astron. Soc., 294, 429-438, (1998).

ADS: http://adsabs.harvard.edu/abs/1998MNRAS.294..429D. 3.2

[47] Di Salvo, T., Goldoni, P., Stella, L., van der Klis, M., Bazzano, A., Burderi, L., Farinelli, R., Frontera, F., Israel, G.L., Méndez, M., Mirabel, I.F., Robba, N.R., Sizun, P., Ubertini, P., and Lewin, W.H.G., "A Hard X-Ray View of Scorpius X-1 with INTEGRAL: Nonthermal Emission?", Astrophys. J. Lett., 649, L91-L94, (2006). Related online version (cited on 24 July 2007): http://arXiv.org/abs/astro-ph/0608335. 4.2.2

[48] Di Salvo, T., Robba, N.R., Iaria, R., Stella, L., Burderi, L., and Israel, G.L., "Detection of a Hard Tail in the X-Ray Spectrum of the Z Source GX 349+2", Astrophys. J., 554, 49-55, (2001).

ADS: http://adsabs.harvard.edu/abs/2001ApJ . .554 . .49D. 4.2 .2

[49] Dolgov, A.D., and Kawasaki, M., "Can modified gravity explain accelerated cosmic expansion?", Phys. Lett. B, 573, 1-4, (2003). 5

[50] Donoghue, J.F., "General relativity as an effective field theory: The leading quantum corrections", Phys. Rev. D, 50, 3874-3888, (1994). Related online version (cited on 24 July 2007): http://arXiv.org/abs/gr-qc/9405057. 2, 5 
Probes and Tests of Strong-Field Gravity with Observations in the Electromagnetic Spectrum 51

[51] Dvali, G., Gabadadze, G., and Porrati, M., "4D gravity on a brane in 5D Minkowski space", Phys. Lett. B, 485, 208-214, (2000).

ADS: http://adsabs.harvard.edu/abs/2000PhLB . 485 . .208D. 2

[52] Dvali, G., Gabadadze, G., and Porrati, M., "A comment on brane bending and ghosts in theories with infinite extra dimensions", Phys. Lett. B, 484, 129-132, (2000).

ADS: http://adsabs.harvard.edu/abs/2000PhLB . 484 . .129D. 2

[53] Dvali, G., Gabadadze, G., and Porrati, M., "Metastable gravitons and infinite volume extra dimensions", Phys. Lett. B, 484, 112-118, (2000).

ADS: http://adsabs.harvard.edu/abs/2000PhLB . 484 . .112D. 2

[54] Eardley, D.M., "Observable effects of a scalar gravitational field in a binary pulsar", Astrophys. J. Lett., 196, L59-L62, (1975).

ADS: http://adsabs.harvard.edu/abs/1975ApJ ...196L . .59E. 3.3, 6.1

[55] Fabian, A.C., Rees, M.J., Stella, L., and White, N.E., "X-ray fluorescence from the inner disc in Cygnus X-1", Mon. Not. R. Astron. Soc., 238, 729-736, (1989).

ADS: http://adsabs. harvard.edu/abs/1989MNRAS.238. .729F. 4.3.2

[56] Fabian, A.C., and Vaughan, S., "The iron line in MCG-6-30-15 from XMM-Newton: evidence for gravitational light bending?", Mon. Not. R. Astron. Soc., 340, L28-L32, (2003).

ADS: http://adsabs.harvard.edu/abs/2003MNRAS.340L..28F. 4.3.2

[57] Falcke, H., Melia, F., and Agol, E., "Viewing the Shadow of the Black Hole at the Galactic Center", Astrophys. J. Lett., 528, L13-L16, (2000).

ADS: http://adsabs.harvard.edu/abs/2000ApJ ...528L. . 13F. 4.1

[58] Flanagan, É.É., and Hughes, S.A., "The basics of gravitational wave theory", New J. Phys., 7, 204, (2005). Related online version (cited on 24 July 2007):

http://arXiv.org/abs/gr-qc/0501041. 4, 7

[59] Gammie, C.F., "Efficiency of Magnetized Thin Accretion Disks in the Kerr Metric", Astrophys. J. Lett., 522, L57-L60, (1999).

ADS: http://adsabs.harvard.edu/abs/1999ApJ ...522L. . 57G. 4.2.3, 4.2.3

[60] Gammie, C.F., McKinney, J.C., and Tóth, G., "HARM: A Numerical Scheme for General Relativistic Magnetohydrodynamics", Astrophys. J., 589, 444-457, (2003).

ADS: http://adsabs .harvard.edu/abs/2003ApJ . . .589 . .444G. 4.2.3

[61] Garcia, M.R., McClintock, J.E., Narayan, R., Callanan, P., Barret, D., and Murray, S.S., "New Evidence for Black Hole Event Horizons from Chandra", Astrophys. J. Lett., 553, L47-L50, (2001).

ADS: http://adsabs .harvard.edu/abs/2001ApJ . . .553L. .47G. 4.2.1

[62] MPI for Gravitational Physics (Albert Einstein Institute), "GEO 600: The German-British Gravitational Wave Detector", project homepage. URL (cited on 05 July 2007): http://geo600.aei.mpg.de. 1, 7

[63] George, I.M., and Fabian, A.C., "X-ray reflection from cold matter in active galactic nuclei and X-ray binaries", Mon. Not. R. Astron. Soc., 249, 352-367, (1991).

ADS: http://adsabs .harvard.edu/abs/1991MNRAS. 249..352G. 4.3.2 
[64] Gierliński, M., Maciołek-Niedźwiecki, A., and Ebisawa, K., "Application of a relativistic accretion disc model to X-ray spectra of LMC X-1 and GRO J1655-40", Mon. Not. R. Astron. Soc., 325, 1253-1265, (2001).

ADS: http://adsabs.harvard.edu/abs/2001MNRAS.325.1253G. 4.2.3

[65] Gierliński, M., Zdziarski, A.A., Poutanen, J., Coppi, P.S., Ebisawa, K., and Johnson, W.N., "Radiation mechanisms and geometry of Cygnus X-1 in the soft state", Mon. Not. R. Astron. Soc., 309, 496-512, (1999).

ADS: http://adsabs.harvard.edu/abs/1999MNRAS.309. .496G. 4.2 .2

[66] Glampedakis, K., and Babak, S., "Mapping spacetimes with LISA: inspiral of a test body in a 'quasi-Kerr' field", Class. Quantum Grav., 23, 4167-4188, (2006). Related online version (cited on 24 July 2007):

http://arXiv.org/abs/gr-qc/0510057. 5

[67] Green, M.B., Schwarz, J.H., and Witten, E., Superstring Theory, Cambridge Monographs on Mathematical Physics, (Cambridge University Press, Cambridge, U.K.; New York, U.S.A., 1988), corr. edition. 2

[68] Grove, J.E., Johnson, W.N., Kroeger, R.A., McNaron-Brown, K., Skibo, J.G., and Phlips, B.F., "Gamma-Ray Spectral States of Galactic Black Hole Candidates", Astrophys. J., 500, 899-908, (1998).

ADS: http://adsabs.harvard.edu/abs/1998ApJ...500. .899G. 4.2 .2

[69] Guilbert, P.W., and Rees, M.J., "Cold' material in non-thermal sources", Mon. Not. R. Astron. Soc., 233, 475-484, (1988).

ADS: http://adsabs.harvard.edu/abs/1988MNRAS.233..475G. 4.3 .2

[70] Harada, T., "Neutron stars in scalar-tensor theories of gravity and catastrophe theory", Phys. Rev. D, 57, 4802-4811, (1998). Related online version (cited on 24 July 2007):

http://arXiv.org/abs/gr-qc/9801049. 6.2

[71] Hawking, S.W., "Black Holes in the Brans-Dicke: Theory of Gravitation", Commun. Math. Phys., 25, 167-171, (1972). 3.3, 5

[72] Hubeny, I., and Hubeny, V., "Non-LTE Models and Theoretical Spectra of Accretion Disks in Active Galactic Nuclei", Astrophys. J. Lett., 484, L37-L40, (1997).

ADS: http://adsabs.harvard.edu/abs/1997ApJ . . 484L. . 37H. 4.2 .3

[73] Iwasawa, K., Miniutti, G., and Fabian, A.C., "Flux and energy modulation of redshifted iron emission in NGC 3516: implications for the black hole mass", Mon. Not. R. Astron. Soc., 355, 1073-1079, (2004). Related online version (cited on 24 July 2007): http://arXiv.org/abs/astro-ph/0409293. 4.3.2

[74] NASA GSFC / SAO, "IXO: International X-ray Observatory", project homepage. URL (cited on 14 November 2008): http://ixo.gsfc.nasa.gov/. 4.3.1, 4.3.2, 7

[75] Kaaret, P., Piraino, S., Bloser, P.F., Ford, E.C., Grindlay, J.E., Santangelo, A., Smale, A.P., and Zhang, W., "Strong-Field Gravity and X-Ray Observations of 4U 1820-30", Astrophys. J. Lett., 520, L37-L40, (1999).

ADS: http://adsabs.harvard.edu/abs/1999ApJ...520L. .37K. 4.4.1

[76] Kato, S., "Basic Properties of Thin-Disk Oscillations", Publ. Astron. Soc. Japan, 53, 1-24, (2001). 4.4 .2 
Probes and Tests of Strong-Field Gravity with Observations in the Electromagnetic Spectrum 53

[77] Kluzniak, W., and Wagoner, R.V., "Evolution of the innermost stable orbits around accreting neutron stars", Astrophys. J., 297, 548-554, (1985).

ADS: http://adsabs .harvard.edu/abs/1985ApJ. . .297 . .548K. 4.4.1

[78] Kramer, M., Backer, D.C., Cordes, J.M., Lazio, T.J.W., Stappers, B.W., and Johnston, S., "Strong-field tests of gravity using pulsars and black holes", New Astron. Rev., 48, 993-1002, (2004). 7

[79] Krichbaum, T.P., Graham, D.A., Witzel, A., Greve, A., Wink, J.E., Grewing, M., Colomer, F., de Vicente, P., Gomez-Gonzalez, J., Baudry, A., and Zensus, J.A., "VLBI observations of the galactic center source SGR A* at $86 \mathrm{GHz}$ and $215 \mathrm{GHz}$ ", Astron. Astrophys., 335, L106-L110, (1998).

ADS: http://adsabs.harvard.edu/abs/1998A\&A...335L.106K. 4.1

[80] Krolik, J.H., "Magnetized Accretion inside the Marginally Stable Orbit around a Black Hole", Astrophys. J. Lett., 515, L73-L76, (1999).

ADS: http://adsabs.harvard.edu/abs/1999ApJ ...515L. .73K. 4.2.3, 4.2.3

[81] Laor, A., "Line profiles from a disk around a rotating black hole", Astrophys. J., 376, 90-94, (1991).

ADS: http://adsabs.harvard.edu/abs/1991ApJ ...376 ...90L. 4.3.2

[82] Lasota, J.-P., "X-rays from quiescent low-mass X-ray binary transients", Astron. Astrophys., 360, 575-582, (2000).

ADS: http://adsabs.harvard.edu/abs/2000A\&A...360 . .575L. 4.2.1

[83] Lattimer, J.M., and Prakash, M., "Neutron Star Structure and the Equation of State", Astrophys. J., 550, 426-442, (2001).

ADS: http://adsabs.harvard.edu/abs/2001ApJ . . 550 . .426L. 6

[84] Laurent, P., and Titarchuk, L., "The Converging Inflow Spectrum Is an Intrinsic Signature for a Black Hole: Monte Carlo Simulations of Comptonization on Free-falling Electrons", Astrophys. J., 511, 289-297, (1999).

ADS: http://adsabs.harvard.edu/abs/1999ApJ. ..511 . .289L. 4.2.2

[85] Lewin, W.H.G., van Paradijs, J., and Taam, R.E., "X-Ray Bursts", Space Sci. Rev., 62, 223-389, (1993). 4.3.1

[86] Lewin, W.H.G., van Paradijs, J., and Taam, R.E., "Black-Hole Candidates", in Lewin, W.H.G., van Paradijs, J., and van den Heuvel, E.P.J., eds., X-ray Binaries, Cambridge Astrophysics Series, vol. 126, p. 126, (Cambridge University Press, Cambridge, U.K.; New York, U.S.A., 1995). 4.2 .3

[87] Li, L.-X., Zimmerman, E.R., Narayan, R., and McClintock, J.E., "Multitemperature Blackbody Spectrum of a Thin Accretion Disk around a Kerr Black Hole: Model Computations and Comparison with Observations", Astrophys. J. Suppl. Ser., 157, 335-370, (2005).

ADS: http://adsabs .harvard.edu/abs/2005ApJS. . 157 . .335L. 4.2.3

[88] California Institute of Technology, "LIGO Scientific Collaboration", project homepage. URL (cited on 05 July 2007):

http://www.ligo.org. 1, 7

[89] Lo, K.Y., Shen, Z.-Q., Zhao, J.-H., and Ho, P.T.P., "Intrinsic Size of Sagittarius A*: 72 Schwarzschild Radii", Astrophys. J. Lett., 508, L61-L64, (1998).

ADS: http://adsabs.harvard.edu/abs/1998ApJ ...508L. .61L. 4.1 
[90] Maartens, R., "Brane-World Gravity", Living Rev. Relativity, 7, lrr-2004-7, (2004). URL (cited on 05 July 2007):

http: //www.livingreviews.org/lrr-2004-7. 1, 2

[91] McClintock, J.E., Narayan, R., and Rybicki, G.B., "On the Lack of Thermal Emission from the Quiescent Black Hole XTE J1118+480: Evidence for the Event Horizon", Astrophys. J., 615, 402-415, (2004). Related online version (cited on 24 July 2007):

http://arXiv.org/abs/astro-ph/0403251. 6, 4.2.1

[92] McClintock, J.E., and Remillard, R.A., "Black hole binaries", in Lewin, W.H.G., and van der Klis, M., eds., Compact Stellar X-Ray Sources, Cambridge Astrophysics Series, vol. 39, pp. 157-213, (Cambridge University Press, Cambridge, U.K.; New York, U.S.A., 2006). 4.2.1, 4.4 .2

[93] McClintock, J.E., Shafee, R., Narayan, R., Remillard, R.A., Davis, S.W., and Li, L.-X., "The Spin of the Near-Extreme Kerr Black Hole GRS 1915+105”, Astrophys. J., 652, 518-539, (2006). Related online version (cited on 24 July 2007):

http://arXiv.org/abs/astro-ph/0606076. 4.2.3

[94] Méndez, M., "The elusive Innermost Stable Circular Orbit: Now you see it, now you don't”, in Antonelli, L.A., Israel, G.L., Piersanti, L., and Tornambè, A., eds., The Multicoloured Landscape of Compact Objects and their Explosive Origins, Proceedings of a conference held in Cefalù, Sicily, June 11-24, 2006, AIP Conference Proceedings, vol. 924, pp. 563-570, (American Institute of Physics, Melville, U.S.A., 2007). Related online version (cited on 05 July 2007):

http://arXiv.org/abs/astro-ph/0611469. 4.4.1

[95] Méndez, M., van der Klis, M., Ford, E.C., Wijnands, R., and van Paradijs, J., "Dependence of the Frequency of the Kilohertz Quasi-periodic Oscillationson X-Ray Count Rate and Colors in 4U 1608-52", Astrophys. J. Lett., 511, L49-L52, (1999).

ADS: http://adsabs.harvard.edu/abs/1999ApJ...511L. .49M. 4.4.1

[96] Middleton, M., Done, C., Gierliński, M., and Davis, S.W., "Black hole spin in GRS 1915+105", Mon. Not. R. Astron. Soc., 373, 1004-1012, (2006). Related online version (cited on 24 July 2007):

http://arXiv.org/abs/astro-ph/0601540. 4.2.3

[97] Milgrom, M., "A modification of the Newtonian dynamics as a possible alternative to the hidden mass hypothesis", Astrophys. J., 270, 365-370, (1983).

ADS: http://adsabs.harvard.edu/abs/1983ApJ . . 270 . .365M. 3.2

[98] Miller, J.M., "A short review of relativistic iron lines from stellar-mass black holes", Astron. Nachr., 327, 997-1003, (2006). Related online version (cited on 24 July 2007):

http://arXiv.org/abs/astro-ph/0609447. 4.3.2

[99] Miller, J.M., "Relativistic X-Ray Lines from the Inner Accretion Disks Around Black Holes", Annu. Rev. Astron. Astrophys., 45, 441-479, (2007). Related online version (cited on 05 July 2007):

http://arXiv.org/abs/0705.0540. 4.3.2

[100] Miller, M.C., Lamb, F.K., and Psaltis, D., "Sonic-Point Model of Kilohertz Quasi-periodic Brightness Oscillations in Low-Mass X-Ray Binaries", Astrophys. J., 508, 791-830, (1998). ADS: http://adsabs.harvard.edu/abs/1998ApJ . . 508. .791M. 4.4.1, 4.4.1 
Probes and Tests of Strong-Field Gravity with Observations in the Electromagnetic Spectrum 55

[101] Miniutti, G., and Fabian, A.C., "A light bending model for the X-ray temporal and spectral properties of accreting black holes", Mon. Not. R. Astron. Soc., 349, 1435-1448, (2004).

ADS: http://adsabs .harvard.edu/abs/2004MNRAS. 349.1435M. 4.3.2

[102] Misner, C.W., Thorne, K.S., and Wheeler, J.A., Gravitation, (W.H. Freeman, San Francisco, U.S.A., 1973). 2, 2

[103] Montero, P.J., Rezzolla, L., and Yoshida, S., "Oscillations of vertically integrated relativistic tori - II. Axisymmetric modes in a Kerr space-time", Mon. Not. R. Astron. Soc., 354, 10401052, (2004).

ADS: http://adsabs.harvard.edu/abs/2004astro.ph. .7642M. 4.4.2

[104] Nandra, K., George, I.M., Mushotzky, R.F., Turner, T.J., and Yaqoob, T., "ASCA Observations of Seyfert 1 Galaxies. II. Relativistic Iron K alpha Emission", Astrophys. J., 477, 602-622, (1997).

ADS: http://adsabs.harvard.edu/abs/1997ApJ . . .477..602N. 4.3.2

[105] Nandra, K., O’Neill, P.M., George, I.M., Reeves, J.N., and Turner, T.J., "An XMM-Newton survey of broad iron lines in AGN", Astron. Nachr., 327, 1039, (2006). Related online version (cited on 24 July 2007):

http://arXiv.org/abs/astro-ph/0610585. 4.3.2

[106] Narayan, R., Garcia, M.R., and McClintock, J.E., "Advection-dominated Accretion and Black Hole Event Horizons", Astrophys. J. Lett., 478, L79-L82, (1997).

ADS: http://adsabs.harvard.edu/abs/1997ApJ . . 478L . .79N. 6, 4.2.1

[107] Narayan, R., Yi, I., and Mahadevan, R., "Explaining the Spectrum of Sagittarius A* with a Model of an Accreting Black-Hole", Nature, 374, 623-625, (1995). 4.1

[108] NASA, "LISA: Laser Interferometer Space Antenna", project homepage. URL (cited on 05 July 2007):

http://lisa.nasa.gov. 7

[109] Niedźwiecki, A., and Zdziarski, A.A., "Bulk motion Comptonization in black hole accretion flows", Mon. Not. R. Astron. Soc., 365, 606-614, (2006). Related online version (cited on 24 July 2007):

http://arXiv.org/abs/astro-ph/0507579. 4.2.2

[110] Noble, S.C., Leung, P.K., Gammie, C.F., and Book, L.G., "Simulating the emission and outflows of accretion disks", Class. Quantum Grav., 24, 259-274, (2007). Related online version (cited on 18 May 2008):

http://arXiv.org/abs/astro-ph/0507579. 4.1

[111] Nowak, M., and Lehr, D., "Stable oscillations of black hole accretion discs", in Abramowicz, M.A., Björnsson, G., and Pringle, J.E., eds., Theory of Black Hole Accretion Discs, Cambridge Contemporary Astrophysics, pp. 233-253, (Cambridge University Press, Cambridge, U.K.; New York, U.S.A., 1998). Related online version (cited on 24 July 2007):

http://arXiv.org/abs/astro-ph/9812004. 4.4.2

[112] Oppenheimer, J.R., and Snyder, H., "On Continued Gravitational Contraction", Phys. Rev., 56, 455-459, (1939). 2

[113] Özel, F., "Soft equations of state for neutron-star matter ruled out by EXO 0748-676", Nature, 441, 1115-1117, (2006). 4.3.1 
[114] Özel, F., and Di Matteo, T., "X-Ray Images of Hot Accretion Flows", Astrophys. J., 548, 213-218, (2001).

ADS: http://adsabs.harvard.edu/abs/2001ApJ . . 548. .2130. 4.1

[115] Özel, F., and Psaltis, D., "Spectral Lines from Rotating Neutron Stars", Astrophys. J. Lett., 582, L31-L34, (2003).

ADS: http://adsabs.harvard.edu/abs/2003ApJ . . 582L . .310. 4.3 .1

[116] Özel, F., Psaltis, D., and Narayan, R., "Hybrid Thermal-Nonthermal Synchrotron Emission from Hot Accretion Flows", Astrophys. J., 541, 234-249, (2000).

ADS: http://adsabs.harvard.edu/abs/2000ApJ . .541. .2340. 4.1

[117] Pais, A., 'Subtle is the Lord': The Science and Life of Albert Einstein, (Oxford University Press, Oxford, U.K., 1982). 2

[118] Papathanassiou, H., and Psaltis, D., "Photon Scattering by Relativistic Flows in Schwarzschild Spacetimes. I. The Generation of Power-Law Spectra", (2000). URL (cited on 05 July 2007):

http://arXiv.org/abs/astro-ph/0011447. 4.2.2

[119] Parker, L., and Simon, J.Z., "Einstein equation with quantum corrections reduced to second order", Phys. Rev. D, 47, 1339-1355, (1993). Related online version (cited on 24 July 2007): http://arXiv.org/abs/gr-qc/9211002. 5

[120] Payne, D.G., and Blandford, R.D., "Compton scattering in a converging fluid flow. III Spherical supercritical accretion", Mon. Not. R. Astron. Soc., 196, 781-795, (1981).

ADS: http://adsabs.harvard.edu/abs/1981MNRAS.196 . .781P. 4.2 .2

[121] Peebles, P.J., and Ratra, B., "The cosmological constant and dark energy", Rev. Mod. Phys., 75, 559-606, (2003). 2, 5

[122] Perlmutter, S., Gabi, S., Goldhaber, G., Goobar, A., Groom, D.E., Hook, I.M., Kim, A.G., Kim, M.Y., Lee, J.C., Pain, R., Pennypacker, C.R., Small, I.A., Ellis, R.S., McMahon, R.G., Boyle, B.J., Bunclark, P.S., Carter, D., Irwin, M.J., Glazebrook, K., Newberg, H.J.M., Filippenko, A.V., Matheson, T., Dopita, M., and Couch, W.J. (The Supernova Cosmology Project), "Measurements of the Cosmological Parameters Omega and Lambda from the First Seven Supernovae at $z \geq 0.35 "$, Astrophys. J., 483, 565-581, (1997).

ADS: http://adsabs.harvard.edu/abs/1997ApJ . . 483. .565P. 1, 3.2

[123] Psaltis, D., "Compton Scattering in Static and Moving Media. II. System-Frame Solutions for Spherically Symmetric Flows", Astrophys. J., 555, 786-800, (2001).

ADS: http://adsabs.harvard.edu/abs/2001ApJ . .555. .786P. 4.2 .2

[124] Psaltis, D., "Models of quasi-periodic variability in neutron stars and black holes", Adv. Space Res., 28, 481-491, (2001). 4.4.1

[125] Psaltis, D., "Constraining Brans-Dicke Gravity with Millisecond Pulsars in Ultracompact Binaries", (2005). URL (cited on 05 July 2007):

http://arXiv.org/abs/astro-ph/0501234. 5, 14, 6.1

[126] Psaltis, D., "Accreting neutron stars and black holes: a decade of discoveries", in Lewin, W.H.G., and van der Klis, M., eds., Compact Stellar X-Ray Sources, Cambridge Astrophysics Series, vol. 39, pp. 1-34, (Cambridge University Press, Cambridge, U.K.; New York, U.S.A., 2006). 4.2 .1 
Probes and Tests of Strong-Field Gravity with Observations in the Electromagnetic Spectrum 57

[127] Psaltis, D., Belloni, T., and van der Klis, M., "Correlations in Quasi-periodic Oscillation and Noise Frequencies among Neutron Star and Black Hole X-Ray Binaries", Astrophys. J., 520, 262-270, (1999).

ADS: http://adsabs.harvard.edu/abs/1999ApJ. . .520 . .262P. 4.4 .1

[128] Psaltis, D., and Lamb, F.K., "Compton Scattering by Static and Moving Media. I. The Transfer Equation and Its Moments", Astrophys. J., 488, 881-894, (1997).

ADS: http://adsabs.harvard.edu/abs/1997ApJ . . 488. . 881P. 4.2.2

[129] Psaltis, D., and Norman, C., "On the Origin of Quasi-Periodic Oscillations and Broad-band Noise in Accreting Neutron Stars and Black Holes", (2000). URL (cited on 05 July 2007): http://arXiv.org/abs/astro-ph/0001391. 4.4.1, 4.4.1

[130] Psaltis, D., Perrodin, D., Dienes, K.R., and Mocioiu, I., "Kerr Black Holes are not Unique to General Relativity", Phys. Rev. Lett., 100, 091101, 1101, (2008).

ADS: http://adsabs.harvard.edu/abs/2008PhRvL.100i1101P. 3.3, 5

[131] Reeves, J.N., Fabian, A.C., Kataoka, J., Kunieda, H., Markowitz, A., Miniutti, G., Okajima, T., Serlemitsos, P., Takahashi, T., Terashima, Y., and Yaqoob, T., "Suzaku observations of iron lines and reflection in AGN", Astron. Nachr., 327, 1079, (2006). Related online version (cited on 24 July 2007):

http://arXiv.org/abs/astro-ph/0610436. 4.3.2

[132] Reynolds, C.S., and Begelman, M.C., "Iron Fluorescence from within the Innermost Stable Orbit of Black Hole Accretion Disks", Astrophys. J., 488, 109-118, (1997).

ADS: http://adsabs.harvard.edu/abs/1997ApJ . . 488. .109R. 4.3.2

[133] Reynolds, C.S., and Nowak, M.A., "Fluorescent iron lines as a probe of astrophysical black hole systems", Phys. Rep., 377, 389-466, (2003). 4.3.2

[134] Reynolds, C.S., Young, A.J., Begelman, M.C., and Fabian, A.C., "X-Ray Iron Line Reverberation from Black Hole Accretion Disks", Astrophys. J., 514, 164-179, (1999).

ADS: http://adsabs.harvard.edu/abs/1999ApJ...514..164R. 4.3.2

[135] Rezzolla, L., Yoshida, S., and Zanotti, O., "Oscillations of vertically integrated relativistic tori - I. Axisymmetric modes in a Schwarzschild space-time", Mon. Not. R. Astron. Soc., 344, 978-992, (2003).

ADS: http://adsabs.harvard.edu/abs/2003MNRAS.344 . .978R. 4.4.2

[136] Riess, A.G., Filippenko, A.V., Challis, P., Clocchiatti, A., Diercks, A., Garnavich, P.M., Gilliland, R.L., Hogan, C.J., Jha, S., Kirshner, R.P., Leibundgut, B., Phillips, M.M., Reiss, D., Schmidt, B.P., Schommer, R.A., Smith, R.C., Spyromilio, J., Stubbs, C., Suntzeff, N.B., and Tonry, J., "Observational Evidence from Supernovae for an Accelerating Universe and a Cosmological Constant", Astron. J., 116, 1009-1038, (1998).

ADS: http://adsabs.harvard.edu/abs/1998AJ....116.1009R. 1, 3.2

[137] Rowan, S., and Hough, J., "Gravitational Wave Detection by Interferometry (Ground and Space)", Living Rev. Relativity, 3, lrr-2000-3, (2000). URL (cited on 05 July 2007): http://www.livingreviews.org/lrr-2000-3. 4

[138] Ryan, F.D., "Gravitational waves from the inspiral of a compact object into a massive, axisymmetric body with arbitrary multipole moments", Phys. Rev. D, 52, 5707-5718, (1995). 5 
[139] Salgado, M., Sudarsky, D., and Nucamendi, U., "Spontaneous scalarization", Phys. Rev. D, 58, 124003, (1998). Related online version (cited on 24 July 2007):

http://arXiv.org/abs/gr-qc/9806070. 6.2

[140] Sanders, R.H., and McGaugh, S.S., "Modified Newtonian Dynamics as an Alternative to Dark Matter", Annu. Rev. Astron. Astrophys., 40, 263-317, (2002). 3.2

[141] Santiago, D.I., Kalligas, D., and Wagoner, R.V., "Nucleosynthesis constraints on scalartensor theories of gravity", Phys. Rev. D, 56, 7627-7637, (1997). Related online version (cited on 24 July 2007): http://arXiv.org/abs/gr-qc/9706017. 3.2

[142] Scheel, M.A., Shapiro, S.L., and Teukolsky, S.A., "Collapse to black holes in Brans-Dicke theory. II. Comparison with general relativity", Phys. Rev. D, 51, 4236-4249, (1995). Related online version (cited on 24 July 2007):

http://arXiv.org/abs/gr-qc/9411026. 3.3, 5

[143] Schödel, R., Ott, T., Genzel, R., Hofmann, R., Lehnert, M., Eckart, A., Mouawad, N., Alexander, T., Reid, M.J., Lenzen, R., Hartung, M., Lacombe, F., Rouan, D., Gendron, E., Rousset, G., Lagrange, A.-M., Brandner, W., Ageorges, N., Lidman, C., Moorwood, A.F.M., Spyromilio, J., Hubin, N., and Menten, K.M., "A star in a 15.2-year orbit around the supermassive black hole at the centre of the Milky Way", Nature, 419, 694-696, (2002). Related online version (cited on 24 July 2007): http://arXiv.org/abs/astro-ph/0210426. 3.2

[144] Seifert, M.D., "Stability of spherically symmetric solutions in modified theories of gravity", Phys. Rev. D, 76, 064002, (2007). Related online version (cited on 05 July 2007): http://arXiv.org/abs/gr-qc/0703060. 5

[145] Shafee, R., McClintock, J.E., Narayan, R., Davis, S.W., Li, L.-X., and Remillard, R.A., "Estimating the Spin of Stellar-Mass Black Holes by Spectral Fitting of the X-Ray Continuum", Astrophys. J. Lett., 636, L113-L116, (2006). Related online version (cited on 24 July 2007): http://arXiv.org/abs/astro-ph/0508302. 4.2.3

[146] Shakura, N.I., and Sunyaev, R.A., "Black Holes in Binary Systems. Observational Appearance", Astron. Astrophys., 24, 337-355, (1973).

ADS: http://adsabs.harvard.edu/abs/1973A\&A...24..337S. 4.2.3

[147] Shapiro, S.L., and Teukolsky, S.A., Black Holes, White Dwarfs and Neutron Stars: The Physics of Compact Objects, (John Wiley \& Sons, Hoboken, U.S.A., 1983). 3.2

[148] Shen, Z.-Q., Lo, K.Y., Liang, M.-C., Ho, P.T.P., and Zhao, J.-H., "A size of 1AU for the radio source Sgr A* at the centre of the Milky Way", Nature, 438, 62-64, (2005). Related online version (cited on 24 July 2007):

http://arXiv.org/abs/astro-ph/0512515. 5

[149] Simon, J.Z., "Higher-derivative Lagrangians, nonlocality, problems, and solutions", Phys. Rev. D, 41, 3720-3733, (1990). 5

[150] Simon, J.Z., "Stability of flat space, semiclassical gravity, and higher derivatives", Phys. Rev. $D, 43,3308-3316,(1991) .5$

[151] International SKA Project Office (ISPO), "SKA: Square Kilometre Array, the international radiotelescope for the 21st century", project homepage. URL (cited on 05 July 2007): http://www. skatelescope.org/. 7 
Probes and Tests of Strong-Field Gravity with Observations in the Electromagnetic Spectrum 59

[152] Sotani, H., and Kokkotas, K.D., "Probing strong-field scalar-tensor gravity with gravitational wave asteroseismology", Phys. Rev. D, 70, 084026, (2004). Related online version (cited on 24 July 2007):

http://arXiv.org/abs/gr-qc/0409066. 5

[153] Sotani, H., and Kokkotas, K.D., "Stellar oscillations in scalar-tensor theory of gravity", Phys. Rev. D, 71, 124038, (2005). Related online version (cited on 24 July 2007):

http://arXiv.org/abs/gr-qc/0506060. 5

[154] Sotiriou, T.P., and Faraoni, V., " $f(R)$ Theories of Gravity", Rev. Mod. Phys., submitted, (2008). Related online version (cited on 18 May 2008):

http://arXiv.org/abs/0805.1726. 1, 2, 5

[155] Sotiriou, T.P., and Liberati, S., "Metric-affine $f(R)$ theories of gravity", Ann. Phys. (N.Y.), 322, 935-966, (2007). Related online version (cited on 24 July 2007):

http://arXiv.org/abs/gr-qc/0604006. 5

[156] Spergel, D.N., Verde, L., Peiris, H.V., Komatsu, E., Nolta, M.R., Bennett, C.L., Halpern, M., Hinshaw, G., Jarosik, N.C., Kogut, A., Limon, M., Meyer, S.S., Page, L., Tucker, G.S., Weiland, J.L., Wollack, E., and Wright, E.L., "First-Year Wilkinson Microwave Anisotropy Probe (WMAP) Observations: Determination of Cosmological Parameters", Astrophys. J. Suppl. Ser., 148, 175-194, (2003).

ADS: http://adsabs.harvard.edu/abs/2003ApJS..148..175S. 1, 3.2, 3

[157] Stairs, I.H., "Testing General Relativity with Pulsar Timing", Living Rev. Relativity, 6, lrr2003-5, (2003). URL (cited on 05 July 2007):

http://www.livingreviews.org/lrr-2003-5. 1, 6.1

[158] Starobinsky, A.A., "A new type of isotropic cosmological models without singularity", Phys. Lett. B, 91, 99-102, (1980).

ADS: http://adsabs.harvard.edu/abs/1980PhLB...91_..99S. 5

[159] Stella, L., Vietri, M., and Morsink, S.M., "Correlations in the Quasi-periodic Oscillation Frequencies of Low-Mass X-Ray Binaries and the Relativistic Precession Model", Astrophys. J. Lett., 524, L63-L66, (1999).

ADS: http://adsabs.harvard.edu/abs/1999ApJ ...524L . .63S. 4.4.1, 4.4.1

[160] Strohmayer, T.E., "Discovery of a 450 HZ Quasi-periodic Oscillation from the Microquasar GRO J1655-40 with the Rossi X-Ray Timing Explorer", Astrophys. J. Lett., 552, L49-L53, (2001).

ADS: http://adsabs.harvard.edu/abs/2001ApJ. ..552L. .49S. 4.4.2

[161] Strohmayer, T.E., and Bildsten, L., "New views of thermonuclear bursts", in Lewin, W.H.G., and van der Klis, M., eds., Compact Stellar X-Ray Sources, Cambridge Astrophysics Series, vol. 39, pp. 113-156, (Cambridge University Press, Cambridge, U.K.; New York, U.S.A., 2006). Related online version (cited on 24 July 2007):

http://arXiv.org/abs/astro-ph/0301544. 4.3.1

[162] Takahashi, R., "Shapes and Positions of Black Hole Shadows in Accretion Disks and Spin Parameters of Black Holes", Astrophys. J., 611, 996-1004, (2004). Related online version (cited on 24 July 2007):

http://arXiv.org/abs/astro-ph/0405099. 4.1 
[163] National Astronomical Observatory of Japan (NAO), "TAMA: The 300m Laser Interferometer Gravitational Wave Antenna", project homepage. URL (cited on 05 July 2007): http://tamago.mtk.nao.ac.jp/. 1, 7

[164] Tanaka, Y., Nandra, K., Fabian, A.C., Inoue, H., Otani, C., Dotani, T., Hayashida, K., Iwasawa, K., Kii, T., Kunieda, H., Makino, F., and Matsuoka, M., "Gravitationally redshifted emission implying an accretion disk and massive black hole in the active galaxy MCG-6-3015", Nature, 375, 659-661, (1995). 4.3.2

[165] Thorne, K.S., and Dykla, J.J., "Black Holes in the Dicke-Brans Theory of Gravity", Astrophys. J. Lett., 166, L35-L38, (1971).

ADS: http://adsabs.harvard.edu/abs/1971ApJ . . 166L . 35T. 3.3, 5

[166] Thorsett, S.E., and Chakrabarty, D., "Neutron Star Mass Measurements. I. Radio Pulsars", Astrophys. J., 512, 288-299, (1999).

ADS: http://adsabs.harvard.edu/abs/1999ApJ . . 512. .288T. 6.1

[167] Titarchuk, L., Mastichiadis, A., and Kylafis, N.D., "X-Ray Spectral Formation in a Converging Fluid Flow: Spherical Accretion into Black Holes", Astrophys. J., 487, 834-846, (1997). ADS: http://adsabs.harvard.edu/abs/1997ApJ . . 487. . 834T. 4.2 .2

[168] Titarchuk, L., and Zannias, T., "The Extended Power Law as an Intrinsic Signature for a Black Hole", Astrophys. J., 493, 863-872, (1998).

ADS: http://adsabs.harvard.edu/abs/1998ApJ . . 493. .863T. 4.2 .2

[169] Tremaine, S., Gebhardt, K., Bender, R., Bower, G., Dressler, A., Faber, S.M., Filippenko, A.V., Green, R., Grillmair, C.J., Ho, L.C., Kormendy, J., Lauer, T.R., Magorrian, J., Pinkney, J., and Richstone, D.O., "The Slope of the Black Hole Mass versus Velocity Dispersion Correlation", Astrophys. J., 574, 740-753, (2002).

ADS: http://adsabs.harvard.edu/abs/2002ApJ...574. .740T. 4

[170] van der Klis, M., "A Possible Explanation for the "Parallel Tracks" Phenomenon in LowMass X-Ray Binaries", Astrophys. J., 561, 943-949, (2001).

ADS: http://adsabs.harvard.edu/abs/2001ApJ...561..943V. 4.4.1

[171] van der Klis, M., "Rapid X-ray variability", in Lewin, W.H.G., and van der Klis, M., eds., Compact Stellar X-Ray Sources, Cambridge Astrophysics Series, vol. 39, pp. 39-112, (Cambridge University Press, Cambridge, U.K.; New York, U.S.A., 2006). 4.4, 4.4.1, 6.3

[172] van Straten, W., Bailes, M., Britton, M.C., Kulkarni, S.R., Anderson, S.B., Manchester, R.N., and Sarkissian, J.M., "A test of general relativity from the three-dimensional orbital geometry of a binary pulsar", Nature, 412, 158-160, (2001). Related online version (cited on 24 July 2007):

http://arXiv.org/abs/astro-ph/0108254. 6.1

[173] Verbunt, F., "Origin and evolution of X-ray binaries and binary radio pulsars", Annu. Rev. Astron. Astrophys., 31, 93-127, (1993). 4.2 .1

[174] Villarreal, A.R., and Strohmayer, T.E., "Discovery of the Neutron Star Spin Frequency in EXO 0748-676", Astrophys. J., 614, L121-L124, (2004). Related online version (cited on 24 July 2007): http://arXiv.org/abs/astro-ph/0409384. 4.3.1 
Probes and Tests of Strong-Field Gravity with Observations in the Electromagnetic Spectrum 61

[175] Istituto Nazionale di Fisica Nucleare, "VIRGO Project Central Web Site", project homepage. URL (cited on 05 July 2007):

http://www.virgo.infn.it. 1, 7

[176] Wagoner, R.V., "Relativistic diskoseismology", Phys. Rep., 311, 259-269, (1999).

ADS: http://adsabs.harvard.edu/abs/1999PhR ...311_.259W. 4.4.2

[177] Wagoner, R.V., Silbergleit, A.S., and Ortega-Rodríguez, M., “'Stable’ Quasi-periodic Oscillations and Black Hole Properties from Diskoseismology", Astrophys. J., 559, L25-L28, (2001).

ADS: http://adsabs.harvard.edu/abs/2001ApJ . . 559L . .25W. 4.4.2, 12

[178] Wald, R.M., General Relativity, (University of Chicago Press, Chicago, U.S.A., 1984). 2, 4.4.2

[179] Weinberg, S., "The cosmological constant problem", Rev. Mod. Phys., 61, 1-23, (1989). ADS: http://adsabs.harvard.edu/abs/1989RvMP ...61...1W. 1

[180] Will, C.M., Theory and experiment in gravitational physics, (Cambridge University Press, Cambridge, U.K.; New York, U.S.A., 1993), 2nd edition. 5, 6

[181] Will, C.M., "The Confrontation between General Relativity and Experiment", Living Rev. Relativity, 9, lrr-2006-3, (2006). URL (cited on 05 July 2007):

http://www.livingreviews.org/lrr-2006-3. 1, 2, 2

[182] Will, C.M., and Zaglauer, H.W., "Gravitational radiation, close binary systems, and the Brans-Dicke theory of gravity", Astrophys. J., 346, 366-377, (1989).

ADS: http://adsabs.harvard.edu/abs/1989ApJ ...346. .366W. 3.3, 5, 6.1

[183] Wilms, J., Reynolds, C.S., Begelman, M.C., Reeves, J.N., Molendi, S., Staubert, R., and Kendziorra, E., "XMM-EPIC observation of MCG-6-30-15: direct evidence for the extraction of energy from a spinning black hole?", Mon. Not. R. Astron. Soc., 328, L27-L31, (2001).

ADS: http://adsabs.harvard.edu/abs/2001MNRAS .328L. .27W. 9

[184] Woodard, R.P., "Avoiding Dark Energy with 1/R Modifications of Gravity", (2006). URL (cited on 05 July 2007):

http://arXiv.org/abs/astro-ph/0601672. 1, 5

[185] European Space Agency (ESA), "XEUS: The X-Ray Evolving Universe Spectrometer", project homepage. URL (cited on 05 July 2007):

http://www.rssd.esa.int/index.php?project=XEUS. 4.3.1, 4.3.2, 4.4.2, 7

[186] Yuan, F., Quataert, E., and Narayan, R., "Nonthermal Electrons in Radiatively Inefficient Accretion Flow Models of Sagittarius A*", Astrophys. J., 598, 301-312, (2003).

ADS: http://adsabs.harvard.edu/abs/2003ApJ...598. .301Y. 4.1

[187] Zhang, S.N., Cui, W., and Chen, W., "Black Hole Spin in X-Ray Binaries: Observational Consequences", Astrophys. J. Lett., 482, L155-L158, (1997).

ADS: http://adsabs.harvard.edu/abs/1997ApJ . . 482L.155Z. 4.2.3

[188] Zhang, W., Smale, A.P., Strohmayer, T.E., and Swank, J.H., "Correlation between Energy Spectral States and Fast Time Variability and Further Evidence for the Marginally Stable Orbit in 4U 1820-30", Astrophys. J. Lett., 500, L171-L174, (1998).

ADS: http://adsabs.harvard.edu/abs/1998ApJ...500L.171Z. 10, 4.4.1 